\title{
Strategic voting and social welfare rules
}

Citation for published version (APA):

Ergin, E. (2018). Strategic voting and social welfare rules. [Doctoral Thesis, Maastricht University]. Maastricht University. https://doi.org/10.26481/dis.20181219ee

Document status and date:

Published: 01/01/2018

DOI:

10.26481/dis.20181219ee

Document Version:

Publisher's PDF, also known as Version of record

\section{Please check the document version of this publication:}

- A submitted manuscript is the version of the article upon submission and before peer-review. There can be important differences between the submitted version and the official published version of record.

People interested in the research are advised to contact the author for the final version of the publication, or visit the DOI to the publisher's website.

- The final author version and the galley proof are versions of the publication after peer review.

- The final published version features the final layout of the paper including the volume, issue and page numbers.

Link to publication

\footnotetext{
General rights rights.

- You may freely distribute the URL identifying the publication in the public portal. please follow below link for the End User Agreement:

www.umlib.nl/taverne-license

Take down policy

If you believe that this document breaches copyright please contact us at:

repository@maastrichtuniversity.nl

providing details and we will investigate your claim.
}

Copyright and moral rights for the publications made accessible in the public portal are retained by the authors and/or other copyright owners and it is a condition of accessing publications that users recognise and abide by the legal requirements associated with these

- Users may download and print one copy of any publication from the public portal for the purpose of private study or research.

- You may not further distribute the material or use it for any profit-making activity or commercial gain

If the publication is distributed under the terms of Article $25 \mathrm{fa}$ of the Dutch Copyright Act, indicated by the "Taverne" license above, 


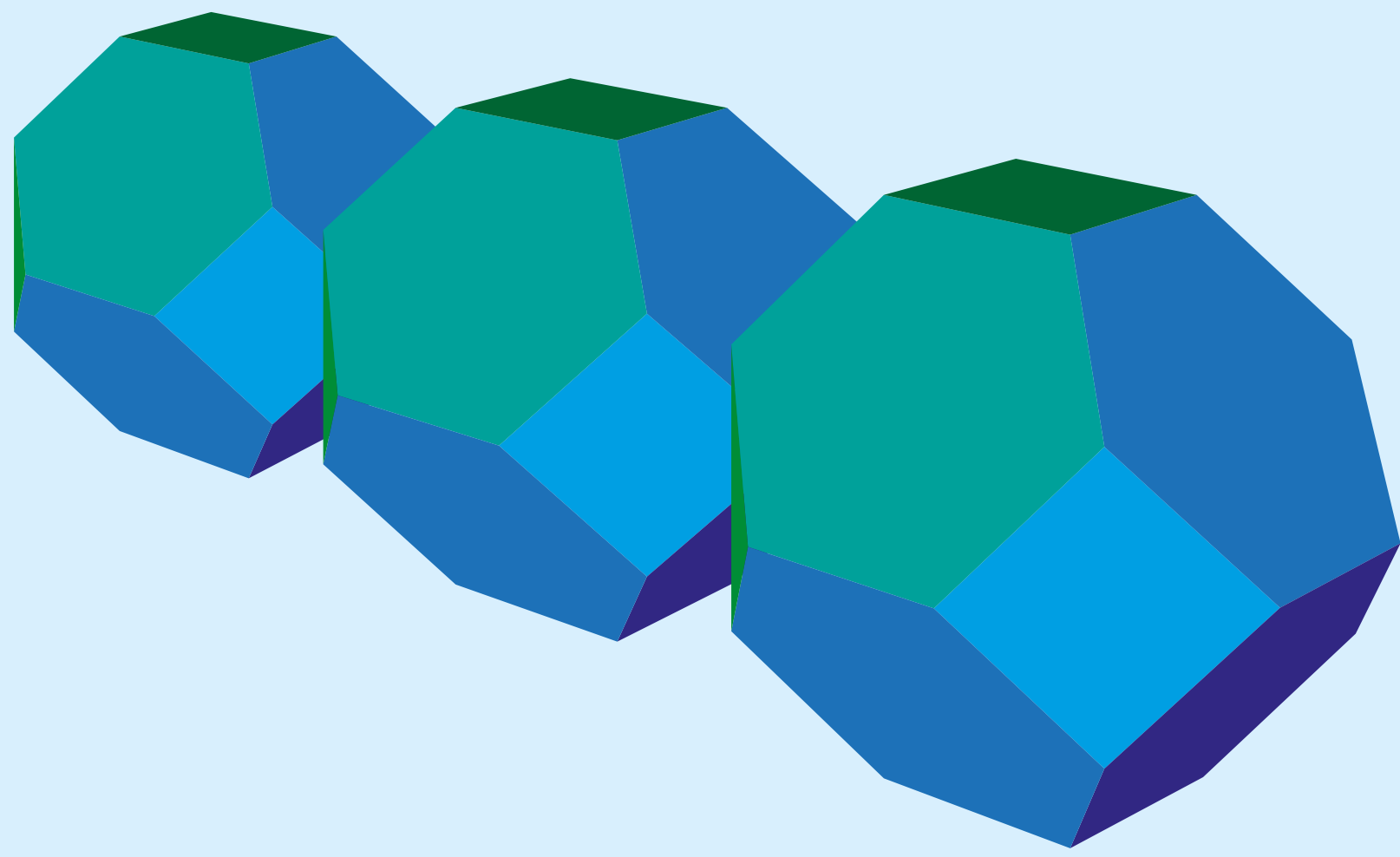

Strategic Voting and Social Welfare Rules

Emre Ergin 



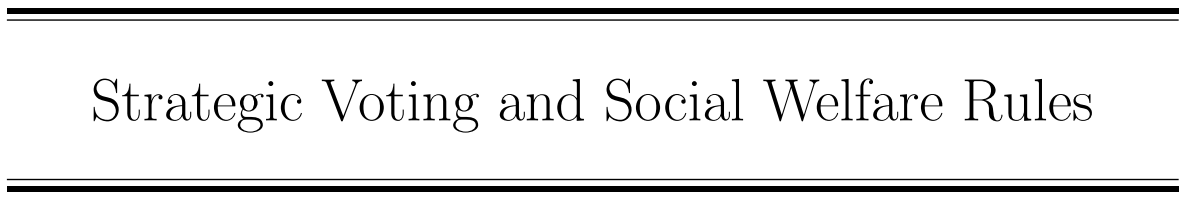

Netherlands, 2018

Written by

EMRE ERGIN

Maastricht University 
(C) copyright Emre Ergin, Maastricht 2018

All rights reserved. No part of this publication may be reproduced, stored in a retrieval system or transmitted, in any form or by any means, electronic, mechanical, photocopying, recording or otherwise, without prior permission of the author or the copyright-owning journals for previous published chapters.

This book was typeset by the author using $\mathrm{AT}_{\mathrm{E}} \mathrm{X}$. Cover design by Elif Sena Ergin.

ISBN: 978-94-6380-152-2

Printing: ProefschriftMaken || www.proefschriftmaken.nl 


\title{
Strategic Voting and Social Welfare Rules
}

\author{
DISSERTATION
}

to obtain the degree of Doctor at the Maastricht University, on the authority of the Rector Magnificus,

Prof. dr. Rianne M. Letschert

in accordance with the decision of the Board of Deans,

to be defended in public

on Wednesday $19^{\text {th }}$ December 2018 at 17:00 hours

by

Emre Ergin 


\section{Supervisor}

Prof. Dr. Arno Riedl

\section{Co-supervisors}

Prof. Dr. Hans Peters

Dr. Burak Can

\section{Assessment Committee}

Prof. Dr. P. Jean-Jacques Herings (chairman)

Dr. Kristof Bosmans

Prof. Dr. Ad van Deemen (Radboud University, Nijmegen)

Prof. Dr. M. Remzi Sanver (Université Paris-Dauphine, France) 
To my wife and my parents. 



\section{Acknowledgments}

My years in Maastricht have been an invaluable experience. One would only think that I would learn things related to research here, but it was much more than that. Not only I learned how to be a researcher, how to be a coauthor, how to be a presenter, but also how to be a tutor, how to be an employee and how to be an office mate. It does not stop even there. I also learned how to be an expat, how to be an author (with different meanings of this word), how to be a tenant, and most importantly, how to be a husband. My years here coincided with absolutely important events and steps in my life, and will definitely be memorable.

I should first start by thanking Burak Can for making these years here possible. He was a constant reminder of the value for practical uses of research and encouraged me to see the bigger picture. I am also thankful to Arno Riedl for his time, his valuable comments, and being always helpful whenever I may be in need. Lastly, I thank Hans Peters, for taking over after Burak's leave, and helping me to shape the question I have into a formal inquiry. I also thank department secretary Elke Lucas for providing me a good working environment with her always kind and helpful attitude.

I am thankful to GSBE for the financial support in general and also for the International Travel Grant that made my visit to Budapest, Corvinus University happen. I am thankful to my co-author Péter Csóka for hosting me there. I was also really happy to meet another co-author, Mohsen Pourpoune, during his visit to Maastricht University. In its essence, the international environment I worked in was helpful in every step along the way. 
Most of this thesis has been written in a single room: A.107. Being with like-minded people motivated me to work harder. I always felt a part of the amazing group of young academicians I shared the office with. I thank Hande for answering the countless questions I had. I thank Evy for her always kind attitude in the rare events we both were in the office. Péter has been a good friend to me not only many common interests but also with some differences made our discussions into fruitful debates. Thank you for beating me in chess. Giang, I am sorry that I somehow made you jump out of fright each time I entered the room. I hope that taking over my desk will help you in that.

As an introvert, I took my sweet time to get rid of my shell. First attempt was made by Zsombor, by introducing me to a new world of board games. Thanks for teaching me how to play Pandemic and Dominant Species. After Zsombor's leave, it took a while for me to seek company. Once I did, my office mates were there for me. Our board gaming and pool nights were really fun. Thank you for organizing those, Péter. I have met with Anastas and Jasmine in those. Thank you Anastas for inviting me to $M L S E$ numerous times, and sorry for taking so long to settle a date. Thank you Jasmine for being generally on the same team with me while playing pool.

One of the most fitting words to describe my four and a half years here would be: "homesick". Thankfully, Maastricht had a big local Turkish community, within and outside of academia. I am thankful to have Kutay, Merve, Seher, Toygar, Çiğdem, Zafer and Ayşe here as colleagues, they were always so nice to talk with. I have met Onur, Burcu, Deniz at our dinners together as "Turkish community", and I am really glad for that. I also need to mention many local immigrants that were here for a long, long time. Ahmet, Bayram and Sefer "Ağabey"s, and Nesrin, Nezahat "Abla"s were almost uncle and auntlike for me, being there whenever a need arises.

I was definitely not the first Turkish friend Inge had, but she is the first Dutch friend of mine. Thanks Inge, for being always pleasant to talk with.

My biggest gratitude goes to my family. Both the original family I was born in, and the new family I started during my years here. My father was always a nice example with his discipline and taught me to live with principles. My mother taught me how to be curious, and was interested whatever silly thing I 
came up with since my childhood. My sister, Elif is a treasure I cherish with all my heart, her laughter echoes in my ears always. And my wife Sena ${ }^{1} \ldots$ She was not even my fiancée when I first started my job here, but even then she was the greatest support one can ever ask for. She was with me and standing by me, first from 2600 kilometers afar, then within the 45 square meters we shared. This $\mathrm{PhD}$ was not an easy journey for me, but thanks to my families, I did not carry the burden alone. Thus, this thesis is dedicated to them.

\footnotetext{
${ }^{1}$ There are many footnotes all around the thesis, which is not visible to the naked eye, mentioning how much I love her. I intentionally left this one visible.
} 



\section{Contents}

1 Introduction 1

2 How to choose a non-manipulable delegation? $\quad 7$

2.1 Introduction . . . . . . . . . . . . . . . 7

2.2 Basic notation and preliminary results . . . . . . . . . . . 12

2.2.1 Model . . . . . . . . . . . . . . . . 12

2.2.2 Conditions . . . . . . . . . . . . . . . . 14

2.3 Using thresholds for delegation rules . . . . . . . . . . . . . . . 19

2.4 Characterization of the threshold delegation rules . . . . . . . 25

2.4.1 Delegates and their support in the society . . . . . . . 26

2.4.2 When to choose a delegate, and when not to? . . . . . 28

2.5 Conclusion ................... 30

3 Condorcet versus participation criterion 33

3.1 Introduction . . . . . . . . . . . . . . . . . . . . 33

3.2 Model . . . . . . . . . . . . . . . 35

3.3 Extension of the Criteria to Social Welfare Rules . . . . . . . . 36

3.3.1 Social Choice Rules . . . . . . . . . . . . 37

3.3.2 Social Welfare Rules . . . . . . . . . . . . . . 37

3.3.3 Strong Criteria on Social Welfare Rules . . . . . . . . . 38

3.3.4 Weak Criteria on Social Welfare Rules . . . . . . . . . 39

3.4 Results. . . . . . . . . . . . . . . . . . . 40

3.5 Specific Rules .................... 43 
3.5.1 Copeland rule . . . . . . . . . . . . . . . . . 44

3.5.2 Minimax rule . . . . . . . . . . . . . . . . . . . 44

3.5 .3 Scoring rules $\ldots \ldots \ldots \ldots \ldots$

3.5 .4 Kemeny rule . . . . . . . . . . . . . . . . 46

3.5.5 Slater rule . . . . . . . . . . . . . . . . . . 46

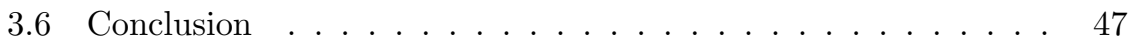

4 How to compare rulers $\quad 49$

4.1 Introduction . . . . . . . . . . . . . . . . . . . . 49

4.2 Model . . . . . . . . . . . . . . . . . . . . 53

4.2 .1 General Framework . . . . . . . . . . . . . 53

$4.2 .2 \quad$ Focus In This Chapter . . . . . . . . . . . . . . . 55

4.3 Expected Utility as a Semi-Metric . . . . . . . . . . . . . 60

4.4 Comparison with Kemeny Distance . . . . . . . . . . . . 70

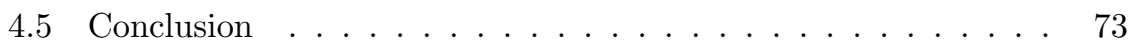

$\begin{array}{ll}\text { Bibliography } & 75\end{array}$

$\begin{array}{ll}\text { Appendices } & 83\end{array}$

$\begin{array}{ll}\text { Valorisation } & 107\end{array}$

$\begin{array}{ll}\text { Curriculum Vitae } & 111\end{array}$ 


\section{List of Figures}

Threshold Rule Example 1 . . . . . . . . . . . . . . . . . 22

Threshold Rule Example $2 \ldots \ldots \ldots$. . . . . . . . . . . . . . . . . . . . . 23

Threshold Rule Example 3 . . . . . . . . . . . . . . . . . 24

Threshold Rule Example 4 . . . . . . . . . . . . . . . 25

3.1 Summary of Section $3.4 \ldots \ldots \ldots \ldots$ 



\section{List of Tables}

3.1 Summary of Section $3.5 \ldots \ldots \ldots \ldots \ldots$

4.1 Lotteries for $A=\{a, b\} \ldots \ldots \ldots \ldots \ldots \ldots \ldots$

4.2 Some exemplary lotteries for $|A|=3 \ldots \ldots \ldots \ldots \ldots$ 



\section{Chapter 1}

\section{Introduction}

Rational choice is a central concept in economics. It comes with two main assumptions, completeness, i.e., a rational agent will always be able to compare two different options, and transitivity, i.e, if a rational agent prefers $a$ to $b$ and $b$ to $c$ then he will prefer $a$ to $c$. The rationality assumption implies that every agent has an order across all possible options. As an example, consider the preference order of a child that is considering different fruits to eat. Possible alternatives are: (A)pples, (B)ananas and (C)herries, and the child prefers apples to cherries and cherries to bananas. The implied order will be $A \succ C \succ B$, which could be further shortened into $A C B$.

This, in general is an example of how economists view people in the society. Without any specification about what the agents will choose, they assume these minimal consistency axioms to be able to build a model that is used to understand decision making.

It is less straightforward to use this intuition when modeling decision making by a group of people. We can still assume some rankings over the issues they are trying to choose from, but how to move from individual rankings to a collective one is a more complex problem. Following from the previous example, let us expand the example by looking at the decision by a family on which fruit to eat. As before, the child has the preference $A C B$, while the mother of the family has the preference $C A B$ and the father has the preference of 
$B A C$. Social choice theory is interested in how to move from these individual preferences to a collective result.

Social choice theory is generally assumed to be founded by the seminal work Arrow (1951), although how to aggregate preferences by the society into a collective decision was first dealt with de Borda (1781); Condorcet (1785) in a less formal way. The main goal of the literature is analyzing ways to aggregate preferences as mentioned and checking whether these methods of aggregation satisfy some reasonable axioms.

All of the three chapters in this dissertation deal with social choice theory. Furthermore, these three chapters specifically deal with social welfare rules. The defining property of this concept is that the aggregation procedure will result in some ranking over the same alternatives that the agents rank. Following from the example above, for the family with the given preferences, order $C A B$ might be the result of a social welfare rule. This is interpreted as, collectively the family would prefer to eat cherries over apples, and apples over bananas.

Note that, coincidentally, the result of such a procedure may give rise to a ranking exactly equal to one of the rankings that joins the aggregation. For example, after using an aggregation method, the family above may reach the aggregate ranking of $C A B$ which is the same ranking as the mother. Note that, in this instance, the family, as an aggregate, compares different alternatives in the same way the mother does. Effectively, it is as if the mother was the one that decides on behalf of the family. This may be interpreted as social welfare rules aim to create a virtual representative, formalized as a ranking, that will be hypothetically treated as an individual decision maker, even if there is no person that has that exact ranking in the society.

A thorough analysis of the concept of representation is outside the scope of this dissertation. For that, we refer the reader to Pitkin (1967). Still, here we will briefly mention the nuances in chapters about views on representation. Descriptive representation, which suggests that representatives represent the society in the way that maps represent the land it pictures, is implicitly assumed in Chapters 2 and 3. In those chapters, we consider agents that only care about their own representation, independent of what other rankings are included in the set that represents the society. This assumes an intrinsic value of 
transferring the information one has about their preferences to the public, or making their voices heard. However, in Chapter 4, we actually focus on a more direct understanding of representation. After some ranking is chosen to represent the society, then the representative, (named "ruler" in the title of that chapter) acts on behalf of the society, which in turn provides some benefit for the voters.

\section{Outline of the Chapters 2-4}

\section{Chapter 2}

The first chapter deals with forming a delegation from a society that ranks some issues. Interpreted as delegations, social welfare rules that are used will be naturally assumed to be non-decisive, that is, there may be more than one ranking in the outcome. Given this framework, the first chapter presents a characterization result. Here, we include an informal summary of the conditions, which will be improved upon in Chapter 2 .

- Pareto efficiency: If there is unanimous agreement on some alternative $a$ beating $b$, then all of the rankings in the outcome will agree with this.

- Consistency: If two sets of voters agrees on a delegate, and if they are merged, the delegation will still be the same.

- Ballot neutrality: Knowing the support of the distribution of the votes will be enough for finding the delegation.

- Strategy-proofness: The distance between the original ranking of some voter and the closest delegate can not be improved by dishonest voting.

These four axioms then are shown to characterize a class of rules which we call threshold rules. Threshold rules are, by definition, very inclusive, that is, resulting delegations will include most of the rankings. There is a large number of cases in which threshold rules give the universal set as the delegation, that is any voter should be exactly represented in the delegation. This may be 
interpreted as an argument for inclusivity in delegations, that is to satisfy these reasonable properties, we must invite all relevant parties to the table. On the other hand, especially in large domains where there are too many possible rankings, including everyone in the delegation may not be feasible. Then, another interpretation may be that these axioms are compatible only when such concerns about feasibility can be negligible, which brings the discussion closer to an impossibility result.

\section{Chapter 3}

While Chapter 2 focuses on strategic voting by misreports, Chapter 3 focuses on another kind of strategic voting: the decision to vote, or to abstain. The Participation criterion is one of the two main concepts this chapter is interested in. The other concept is called Condorcet criterion. Again, we include here an informal definition of the concepts that will be improved upon in Chapter 3.

- Participation Criterion: No agent should be better off by not joining the election.

- Condorcet Criterion: The outcome should respect all one-on-one pairwise votings, (like voting first between $A$ and $B$, then $B$ and $C$, then $A$ and $C$ and so on) if these votings conform to some consistency between them.

Of course, while the main idea is the same, there are many ways to formalize the intuition given above. In this chapter, we select a few of those to understand the general structure. We still focus on a delegation-like outcome for the social welfare rules, this means, agents compare possibly non-singleton sets of outcomes to decide whether or not to join the election.

The main topic of this chapter is analyzing whether or not there is an incompatibility between these two concepts when using social welfare rules. Moulin (1988) proves the existence of this incompatibility with social choice rules. We also show an impossibility, similar to that result. However, using a weaker axiom for defining participation constraint, we escape from this impossibility, and show that Kemeny rule is included in the class that is 
characterized by the Condorcet criterion, and this weaker participation criterion. Lastly, we list some well-known social welfare rules, and check whether they satisfy the conditions defined in this chapter.

\section{Chapter 4}

Strategic voting is somewhat central in Chapter 2 and Chapter 3. Any effort that seeks to model strategic voting in the context of social welfare rules should include a tool to model preferences over sets of rankings. In these previous chapters, the Kemeny distance (Kemeny, 1959) is used to order different rankings to check whether a manipulation or an abstention is beneficial for the agent. While the use of distances is somehow justified, and common enough in the literature, in the last chapter of this dissertation, we try to build a new way to model preferences over rankings.

We approach the problem by interpreting the outcome of the election as some hypothetical person, named "dictator" in the title of the chapter. In a similar way to revealed preference theory, alternatives other than the topmost one, thus their ranking, only becomes relevant when the topmost alternative is not feasible, not available for the selection. With this in mind, we model the availability of the alternatives as an uncertain event. Then to model the preference over rankings, we introduce two axioms:

- Willingness to Choose: Any non-empty state is preferred to the empty state with no alternatives available.

- Ruler Sovereignty: Agents compare different non-empty states by comparing what the "dictator" would choose in each of them.

Using these two axioms, together with von Neumann-Morgenstern expected utility framework, we introduce expected utility of being subject to a ranking, interpreted as ranking of a ruler. Following from that, a dissimilarity function that is built by the decrease in this expected utility, compared to one's their own ranking is introduced. Then, this dissimilarity function is analyzed to see whether it satisfies properties of a metric, and if so, under what conditions. 
The main result of this chapter suggests that given our interpretation of rankings, using a metric is not justifiable at all, whereas use of semi-metrics is justified under a limited set of parameters. This incompatibility then is made more concrete by a comparison with the suggested method and using the well-known Kemeny distance. 


\section{Chapter 2}

\section{How to choose a non-manipulable delegation ${ }^{1}$}

\subsection{Introduction}

In many situations, individuals participate in collective decision making via a committee of representatives or delegates, i.e., there is a double-layered aggregation of individual opinions. Consider, for instance, voting for political candidates in elections to represent one's opinion in a parliament. Board decisions in large corporations are also taken collectively via a committee representing different departments albeit not every department is allocated a seat at the board. Correspondingly, peace conferences and negotiations over conflict zones require delegates to be sent by different interest groups or ethnicities involved in a civil war. The choice of which interest group or ethnicity to invite to the conference, however, is not very straightforward - especially not in extremely heterogeneous, polarized or divided societies. In fact, the

\footnotetext{
${ }^{1}$ Based on Can, Csóka, and Ergin (2017).
} 
way in which peace conferences are set also signals the possible effectiveness of these talks. A recent example is the Nationwide Ceasefire Agreement (NCA) in 2015 to end the conflict in Myanmar, which was heavily criticized for not being inclusive. However, a year later in 2016, the following peace conference still did not invite any representative for Myanmar's millions of Rohingya Muslims (Myint and Slodkowski, 2016).

In many settings, delegates representing their interest groups do not necessarily carry a weight in the committee. This is especially the case when the delegation is summoned not to make a decision but to discuss and agree on a memorandum of understanding, or exchange ideas, information, perspectives and perhaps eventually act as an advisory board. For instance, with a peace conference scenario in mind, consider a society and the issues it is facing. Delegates representing various ethnic groups are invited to the table, in the hope to create a mutual understanding. Similarly, consider different interest groups who have diverse opinions on how the city council should allocate the budget across different expenditures, e.g., a public park, a new tunnel, or a citywide educational program. Of course, delegates may represent groups with different powers, and hold a varying degree of support from their supporters. However, the output of such initiatives as the first layer in a multi-layered aggregation typically consists of compiling a report or suggestion to another body which makes the decisions or mediates the peace process.

Thinking of individual opinions (priority orderings), we want to find out what limited set of opinions we could choose to represent this society in a reasonable, fair way. We are not interested in how much relative importance an opinion in the delegation has per se, but only in whether an opinion should be invited to the table. Eventually, we might bring together some people representing those opinions on behalf of possibly very diverse interest groups and hope to achieve a fruitful exchange of information and deliberation within the delegation itself.

This chapter investigates possible mechanisms through which this table can be formed. We require such mechanisms to respect some minimal normative requirements. For instance, the delegation choice should respect unanimous agreements in the society and be consistent in choosing delegates when similar 
societies are merged. We expect it to be neutral in the way it treats the opinions, and also expect it to be non-manipulable, such that the individuals have no gain in misreporting their ideas. We propose all these norms as criteria to choose which opinions should get a seat at the table and then show that there is a unique class of rules which satisfy all these criteria, hence a characterization result. These novel rules are non-trivial and relatively simple to comprehend, making them practically usable to choose a delegation.

We assume individuals have preferences (strict rankings) over some available issues, and those preferences form the preference profile of a society. We formalize the delegation rules as mechanisms that assign a set of rankings (opinions) to each given preference profile. Since the delegation is not necessarily comprised of a single ranking, a delegation rule herein corresponds to a social welfare correspondence instead of a social welfare function. However, we employ the term delegation as it entails a particular interpretation. That is, a delegation, which is a set of rankings, is the collection of preferences that represents the society and should be invited to the table.

We propose some normative conditions on how to choose delegates for a society. We require that if all individuals in a society agree on how to rank one issue over another, the delegation should respect that. This is also known as Pareto optimality. Second, we impose that when two distinct societies represented by identical delegations merge, the merged society should also be represented by the same delegation (Young, 1974, 1975; Smith, 1973), an idea known as Consistency ${ }^{2}$. Our third condition, Ballot neutrality reflects an idea of fairness, and requires that only the ballots of individual opinions should matter in the delegation choice $^{3}$. Finally, we require that no individual can manipulate the choice of delegates to their advantage. This condition is called Strategy-proofness. The first two, Pareto optimality and consistency, are very standard conditions in the literature. In what follows we explain further ballot neutrality and strategy-proofness.

Ballot neutrality imposes neutrality towards "equivalent ballots". Consider

\footnotetext{
${ }^{2}$ Consistency additionally implies that the delegation choice is anonymous, a condition which requires that the names of the individuals do not matter.

${ }^{3}$ Ballot neutrality additionally implies that the delegation choice is neutral, a condition which requires that the names of the issues do not matter.
} 
two societies of equal size, say six individuals, facing three issues, hence six possible preferences. Now represent the opinions in both societies by the number of followers each preference has, e.g., $(\mathbf{3}, 2,1,0,0,0)$ and $(0,1,2, \mathbf{3}, 0,0)$. As it happens, the two societies have "equivalent" ballots, i.e., the support distribution is merely a shuffling of the number of followers. In this case, we require the delegation choice in each society to correspond to the support for the delegates. For instance, if the first preference in the former ballot with a support of 3 is chosen as a delegate in the first society, then the fourth preference in the latter ballot should also be chosen in the second society. Ballot neutrality is also a variable alternative axiom, which necessitates that increasing the number of available issues and preferences, does not influence the outcome so long as the distributions of supported opinions are equivalent ${ }^{4}$. We explain this further in detail in the coming section and provide an example in the appendix.

Strategy-proofness requires that the rule is not manipulable by individuals (or coalitions). Therefore a rule being strategy-proof naturally induces honest reporting of individual opinions. Consider a society and a delegation representing it. Suppose an individual misreports his opinion and this alters the delegation such that at least one new delegate is strictly closer ${ }^{5}$ to his opinion than any other delegate in the original delegation. This situation is considered as a successful manipulation. We require that the delegation choice should not be prone to any such manipulation. If a rule is not manipulable by any individual, then we call it individual strategy-proof, whereas if no coalition of individuals can achieve such manipulation, we call it coalitional strategy-proof. The latter is a stronger requirement than the former. Our strategy-proofness concept is substantially different than that of Bossert and Storcken (1992), Bossert and Sprumont (2014) and Athanasoglou (2016), since we allow multiple preferences in the outcome. ${ }^{6}$

\footnotetext{
${ }^{4}$ For instance, increasing the number of issues to 4 , and hence the number of possible preferences to 24 , would still yield the first preference as a delegate if the ballot stayed as $(\mathbf{3}, 2,1,0,0, \ldots, 0)$.

${ }^{5}$ We use the most typical measure of closeness for rankings, i.e., the Kemeny distance (Kemeny, 1959).

${ }^{6}$ When preferences are aggregated into a single alternative, i.e., social choice functions,
} 
We find that there exists a non-dictatorial, non-trivial and in fact, simple class of rules which is characterized by these conditions, and which we call threshold rules. The threshold rules impose different sizes of delegations depending on the composition of the society instead of a fixed size. This is very natural as opinions in a group of people may have a different level of polarization and diversity. Therefore, threshold rules change the size of the delegation depending on the heterogeneity of the society. The rules also share a common lower bound in terms of how much representative power they require for all possible sizes of delegations. For instance, if a delegation is composed of $t$ delegates, then the ratio of individuals whose opinions are not included is always below $(0.5)^{t}$, hence the ratio of the individuals supporting those delegates to the whole society must be strictly higher than $1-0.5^{t}$.

The delegation rules we characterize only differ in "how high" the thresholds are set above the common lower bound. We show that for each threshold rule, there exists a threshold function $f$ which imposes how much minimal support a delegation of size $t$ has to have to be an appropriate representation for a society. The rule orders each possible ranking/delegate according to their support in the society and chooses the lowest number of delegates $t^{*}$ with a total support reaching the respective threshold, i.e., $f\left(t^{*}\right)$. For example, a threshold rule might require $60 \%$ of the society's support for singleton delegations, i.e., $f(1)=0.6$. If this support is not found, then it might look for $85 \%$ of the society's support for a delegation of size $2, f(2)=0.85$. If this support is not found, then the process continues, with monotonically increasing thresholds for each $t$. We show that all threshold rules satisfy two conditions: i) $f(1)>0.5$ and ii) $1 \geq f(t) \geq(f(t-1)+1) / 2$ for $t>2$, i.e., the minimal threshold for a

Gibbard (1973) and Satterthwaite (1975) show the impossibility of finding such proper non-dictatorial and strategy-proof rules. See Barberà (2011) for more on strategy-proof social choice functions. For strategy-proofness of multi-valued social choice rules, see Barberà, Dutta, and Sen (2001). When preferences are aggregated into a single ranking, i.e., social welfare functions, the results are mixed since the definition of strategy-proofness can be quite numerous. Bossert and Storcken (1992) prove an impossibility result, Sato (2013b) offers more positive news, and finally, Bossert and Sprumont (2014) uses a weaker version of strategy-proofness than in Bossert and Storcken (1992) and provides some examples of non-manipulable rules. 
singleton delegation is above $50 \%$, and the minimal threshold for delegations of size $t>2$ is at least the average of the previous level, $f(t-1)$ and $100 \%$, but at most $100 \%$. Of course, $f(1)$ can also start from $100 \%$ (and hence continue at that level), requiring $100 \%$ support for each possible sizes of delegations, which can only be reached by including all the reported preferences. Threshold rules are well-defined, as the threshold for $t$ sooner or later reaches $100 \%$.

As Lanz (2011) argues, "Only stakeholders who add value to the process and augment the chances of reaching a sustainable settlement should be given seats at the table, $[\ldots]$ ". The challenge, therefore, is to make the invitations to the table from a normative perspective while maintaining inclusivity and feasibility. This chapter proposes a quantitative measure on how to arrange the table, the number of seats at the table, and finally how representative in total, the invitees must minimally be.

The chapter proceeds as follows. Section 2.2 presents the notation and conditions. In Section 2.3, we define threshold rules and provide some examples. In Section 2.4, we provide our characterization. Section 2.5 concludes with some policy implication.

\subsection{Basic notation and preliminary results}

\subsubsection{Model}

Let $\mathcal{A}$ be a countably infinite set of alternatives, interpreted as potential issues. Given a finite nonempty subset $A \subsetneq \mathcal{A}$, preferences are taken to be strict priority rankings of these issues, formalized as complete, antisymmetric and transitive binary relations over the set of alternatives $A$. We denote the set of all preferences over $A$ by $\mathcal{L}(A)$. Given a preference $R \in \mathcal{L}(A)$, and two disjoint alternatives $a$ and $b$, the case where $a$ is preferred to $b$ can be denoted by $R=. a . b$. or $(a, b) \in R$. To measure closeness we use the well-known Kemeny distance. ${ }^{7}$ Formally, for two preferences $R_{1}$ and $R_{2}$, the Kemeny distance is $\delta\left(R_{1}, R_{2}\right)=\left(\left|R_{2} \backslash R_{1}\right|+\left|R_{1} \backslash R_{2}\right|\right) / 2$, half of the symmetric set difference.

\footnotetext{
${ }^{7}$ Kemeny (1959) introduced this distance. For a recent, improved characterization, see Can and Storcken (2018).
} 
Let $\mathcal{N}$ be a countably infinite set of agents, interpreted as potential individuals. Given a finite nonempty subset $N \subsetneq \mathcal{N}$ with cardinality $n, \mathcal{L}(A)^{n}$ denotes the set of all preference profiles $P$, i.e., preferences of $n$ agents where $P(i)$ refers to the preference of agent $i \in N$ and $P(S)$ refers to the preference profile, say a subprofile, of a subset of agents $S \subseteq N$. Given a profile $P \in \mathcal{L}(A)^{n}$, and $R \in \mathcal{L}(A)$, we denote the number of agents who reported $R$ in this profile as $p(R)=|\{i \in N \mid P(i)=R\}|$.

Given any finite $A \subsetneq \mathcal{A}$, let $R_{1}, R_{2}, \ldots, R_{|A| !}$ be an enumeration of preferences in $\mathcal{L}(A)$, e.g., the lexicographic enumeration for $A=\{a, b, c\}$ is " $R_{1}=a b c, R_{2}=a c b, R_{3}=b a c, R_{4}=b c a, R_{5}=c a b, R_{6}=c b a$ ". Given any such enumeration, a profile $P \in \mathcal{L}(A)^{n}$ can also be interpreted as a vector composed of the number of followers each preference has, e.g., $p=\left(p_{1}, p_{2}, p_{3}, \ldots, p_{|A| !}\right)$

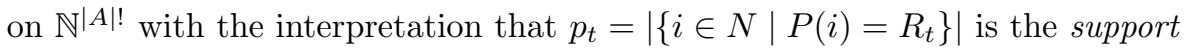
for preference $R_{t} \in \mathcal{L}(A)$ and $p$ is the support for the preference profile $P$. As an example, for 3 alternatives and the enumeration given above, the support for the following preference profile,

$$
P \in \mathcal{L}(A)^{6}=\{\underbrace{a b c, a b c, a b c}_{R_{1}}, \underbrace{b a c, b a c}_{R_{3}}, \underbrace{c a b}_{R_{5}}\}
$$

can be denoted by $p=(3,0,2,0,1,0)$. For simplicity, we also denote the "normalized support" for the same profile similarly, e.g., $p=(0.5,0,0 . \overline{3}, 0,0.1 \overline{6}, 0)$.

Consider two disjoint finite sets of agents $N, N^{\prime}$, and preference profiles $P \in \mathcal{L}(A)^{n}$, and $P^{\prime} \in \mathcal{L}(A)^{n^{\prime}}$. Then, $\bar{P}=\left(P, P^{\prime}\right) \in \mathcal{L}(A)^{n+n^{\prime}}$ denotes the merging of two profiles, i.e, $\bar{P}(i)=P(i)$ if $i \in N$ and $\bar{P}(i)=P^{\prime}(i)$ if $i \in N^{\prime}$. If $P$ and $P^{\prime}$ are such that there exists a bijection $\sigma: N \leftrightarrow N^{\prime}$ such that $P(i)=P^{\prime}(\sigma(i))$ for all $i \in N$, then we call $\bar{P}=\left(P, P^{\prime}\right)$ as a two-fold replica of $P$ and denote it by $2 P$. The definition naturally extends to all $c$-fold replicas $c P$ of $P$, for any $c \geq 2$ for $c \in \mathbb{N}$.

Given any finite $N \subsetneq \mathcal{N}$ and any $A \subsetneq \mathcal{A}$, a delegation rule $\varphi$ is a social welfare correspondence which assigns every preference profile $P \in \mathcal{L}(A)^{n}$ a nonempty subset of preferences $\varphi(P) \subseteq \mathcal{L}(A)$, interpreted as the set of delegates or the delegation for this society. 


\subsubsection{Conditions}

Next, we introduce some conditions on how to choose a delegation. Unless otherwise mentioned, we assume the conditions to hold for all set of alternatives, $A \subsetneq \mathcal{A}$, and for all set of agents, $N \subsetneq \mathcal{N}$. The first condition requires that if everyone prefers an alternative over another, then no delegate should say otherwise.

Definition 2.1. Pareto optimality: A rule $\varphi$ is Pareto Optimal whenever for all $P \in \mathcal{L}(A)^{n}$ and for all $a, b \in A$, if for all $i \in N,(a, b) \in P(i)$, then for all $R \in \varphi(P),(a, b) \in R$.

The second condition we impose concerns merging of two societies each endowed with the same delegation. In such situations, the delegation assigned to the merged society should remain the same. This concept is well known in many contexts under varying names with slight changes, including reinforcement, homogeneity ${ }^{8}$, etc.

Definition 2.2. Consistency: A rule $\varphi$ is consistent whenever for any two disjoint finite sets $N, N^{\prime} \subsetneq \mathcal{N}$ (with cardinality $n$ and $n^{\prime}$ respectively) and for profiles, $P \in \mathcal{L}(A)^{n}$ and $P^{\prime} \in \mathcal{L}(A)^{n^{\prime}}$, if $\varphi(P)=\varphi\left(P^{\prime}\right)$ then $\varphi\left(\left(P, P^{\prime}\right)\right)=$ $\varphi(P)=\varphi\left(P^{\prime}\right)$.

The third condition we impose concerns variable alternative scenarios, wherein the fixed set of individuals face more issues to report their preferences on. Consider, for instance, two sets of alternatives $A \subsetneq \bar{A}$ such that $|A|=3$ and $|\bar{A}|=4$. Consider two profiles on these sets with the following frequency supports:

$$
P \in \mathcal{L}(A)^{n} \text { with } p=(3,2,1,0,0,0) \text { and } \bar{P} \in \mathcal{L}(\bar{A})^{n} \text { with } \bar{p}=(0,1,2,3, \underbrace{0, \ldots, 0)}_{20 \text { entries }}
$$

Note that the nonzero entries in each vector are identical (except for the shuffling). Our condition requires that shuffling the support for preferences

\footnotetext{
${ }^{8}$ Homogeneity is a milder version of this concept, which requires that result would be insensitive to replicating the population (Fishburn, 1977).
} 
should shuffle the delegates in the exact same way. ${ }^{9}$ Formally, given $A \subseteq \bar{A} \subsetneq \mathcal{A}$, a profile $P \in \mathcal{L}(A)^{n}$ and an injection ${ }^{10} \pi:\{1,2, \ldots,|A| !\} \rightarrow\{1,2, \ldots,|\bar{A}| !\}$, we say $\bar{P} \in \mathcal{L}(\bar{A})^{n}$ is an "expansion of $P$ by $\pi$ " if for all $i \in\{1,2, \ldots,|A| !\}$ we have $p_{i}=\bar{p}_{\pi(i)}$. We consider such profiles $P, \bar{P}$ to have equivalent ballots and refer to $\pi$ as a corresponding injection. ${ }^{11}$

Definition 2.3. Ballot neutrality: A rule is ballot neutral whenever for any $P \in \mathcal{L}(A)^{n}, \bar{P} \in \mathcal{L}(\bar{A})^{n}$ with equivalent ballots, and for any corresponding injection $\pi$, we have:

$$
R_{i} \in \varphi(P) \text { if and only if } \bar{R}_{\pi(i)} \in \varphi(\bar{P}) .
$$

We provide an example in Appendix A.2 to illustrate this condition. ${ }^{12}$ Next, we show that ballot neutrality, together with Pareto optimality, implies that the delegation can only be chosen from preferences that are reported. Thus we do not have to worry about finding a delegate whose role would be to represent some "compromised" preference. Let $R P(P)=\{R \in \mathcal{L}(A) \mid p(R)>0\}$, denote the set of reported preferences, preferences which are reported by at least one agent in profile $P$.

Proposition 2.1. If a rule $\varphi$ satisfies Pareto optimality and ballot neutrality, then for all $P \in \mathcal{L}(A)^{n}, \varphi(P) \subseteq R P(P)$.

Proof. The proof is in Appendix A.2.1.

The following remark says that we can always find an expansion for a profile in which delegates and non-delegates are clustered, that is, each agent whose

\footnotetext{
${ }^{9}$ This condition is, in fact, an amalgamation of two well-known conditions, neutrality and anonymity, and stronger than both.

${ }^{10}$ For $A=\bar{A}, \pi$ is a permutation.

${ }^{11}$ There may be more than one corresponding injection for two equivalent ballots.

${ }^{12}$ Note that the definition of ballot neutrality even extends to profiles on two disjoint sets of alternatives. For instance, let $A=\{x, y, z\}$ and $B=\{a, b, c\}$, and consider two profiles $P \in \mathcal{L}(A)^{n}$ and $\bar{P} \in \mathcal{L}(B)^{n}$ with identical ballots. Consider expansions of $P$ and $\bar{P}$, say $P^{\prime}$ and $\bar{P}^{\prime}$ respectively, to $A \cup B$ by some injection. Ballot neutrality applies between $P$ and $P^{\prime}$ (and between $\bar{P}$ and $\bar{P}^{\prime}$ ). By construction, $P^{\prime}$ and $\bar{P}^{\prime}$ have equivalent ballots. Therefore ballot neutrality applies between $P^{\prime}$ and $\bar{P}^{\prime}$. This, in turn, imposes ballot neutrality between $P$ and $\bar{P}$.
} 
preference is not in the delegation will prefer any non-delegate to any delegate. In other words, every agent who supports a preference which is not part of the delegation would like to enlarge the delegation set.

Let us extend our definition of injections to sets of preferences. Given $A \subseteq \bar{A} \subsetneq \mathcal{A}$ and any injection $\pi:\{1,2, \ldots|A| !\} \rightarrow\{1,2, \ldots|\bar{A}| !\}$, and any $X \subseteq \mathcal{L}(A)$,

$$
\pi(X)=\left\{\bar{R}_{\pi(i)} \in \mathcal{L}(\bar{A}) \mid R_{i} \in X\right\} .
$$

Remark 2.1. Note that, since $\mathcal{A}$ is infinite, for any $A \subsetneq \mathcal{A}$, for any preference profile on $A$ and for any two disjoint sets $X, Y \subsetneq \mathcal{L}(A)$, we can always find an expansion $P$ by some $\pi$ of the initial preference profile such that the injections of the two sets $X$ and $Y$ (denoted respectively by $\pi(X)$ and $\pi(Y)$ ), form clusters that are "far away" from each other. Formally:

$$
\max _{R, R^{\prime} \in \pi(X)} \delta\left(R, R^{\prime}\right)<\min _{R \in \pi(X), R^{\prime} \in \pi(Y)} \delta\left(R, R^{\prime}\right)
$$

(The example in Appendix A.3 illustrates how this remark is implemented.)

Our third condition, strategy-proofness, implies that no agent should "benefit" from misreporting his preference, i.e., truth telling is a weakly dominant strategy. We say an agent $i$ weakly prefers a delegate $R_{1}$ to another delegate $R_{2}$, whenever $P(i)$ is weakly closer to $R_{1}$ than it is to $R_{2}$ in terms of the Kemeny distance, i.e., $\delta\left(P(i), R_{1}\right) \leq \delta\left(P(i), R_{2}\right)$. Similarly, we say an agent $i$ weakly prefers a delegation $D_{1}$ to another delegation $D_{2}$, whenever $P(i)$ is weakly closer to the most preferred delegate in $D_{1}$ than it is to the most preferred delegate in $D_{2}$, i.e., $\min \left\{\delta\left(P(i), R_{1}\right) \mid R_{1} \in D_{1}\right\} \leq \min \left\{\delta\left(P(i), R_{2}\right) \mid R_{2} \in D_{2}\right\}$. Strategy-proofness means that every agent weakly prefers the delegation they get under true preferences to any delegation they achieve by misreporting. In other words, there is no possibility of misreporting and getting a new delegate in the delegation which is closer to the agent's preference. Here, we take the closest delegate as the only relevant one for the agents, meaning agents do not care about the distance to other delegates. ${ }^{13}$ We first discuss the usual individual strategy-proofness and afterward the coalitional version of it. In the

\footnotetext{
${ }^{13}$ Here we do not assume any negative externality in representativeness, i.e., agents only
} 
sequel we shall only use the former. However, we show later in Proposition 2.2 that the latter is implied by the former under ballot neutrality.

Definition 2.4. Strategy-proofness: A rule $\varphi$ is strategy-proof whenever for all $i \in N$ and for all $P \in \mathcal{L}(A)^{n}$, there exists no $P^{\prime}=\left(P^{\prime}(i), P(N \backslash\{i\})\right) \in$ $L(A)^{n}$ such that

$$
\min _{R \in \varphi(P)} \delta(P(i), R)>\min _{R \in \varphi\left(P^{\prime}\right)} \delta(P(i), R) .
$$

Definition 2.5. Coalitional strategy-proofness: A rule is coalitional strategy-proof whenever for all coalitions $S \subseteq N$ and for all $P \in \mathcal{L}(A)^{n}$, there exists no $P^{\prime}=\left(P^{\prime}(S), P(N \backslash S)\right) \in \mathcal{L}(A)^{n}$ such that:

$$
\begin{gathered}
\min _{R \in \varphi(P)} \delta(P(i), R)>\min _{R \in \varphi\left(P^{\prime}\right)} \delta(P(i), R) \\
\text { for all } i \in S .
\end{gathered}
$$

Remark 2.2. Note that our individual strategy-proofness concept is substantially different than that of Bossert and Storcken (1992) and of Athanasoglou (2016). We allow multiple preferences in the outcome (in the case of singlevalued delegation rules they are equivalent). Bossert and Sprumont (2014) differs from the former two interpretations since the manipulation is based on a concept known as betweenness (see also Grandmont (1978), Kemeny (1959), and Sato (2013b)). In their interpretation, an agent can benefit only when the outcome is manipulated to somewhere between herself and the preference corresponding to truth telling. In our interpretation agents can benefit when the outcome is manipulated to anywhere, resulting a closer preference. This makes the strategy-proofness we propose, ceteris paribus, stronger and harder to satisfy. We provide an example in Appendix A.1.1 which is strategy-proof in Bossert and Sprumont (2014), but not in the way we interpret it.

Next, we show that under ballot neutrality strategy-proofness implies coalitional strategy-proofness. We use this implication throughout the proofs.

care about the delegate(s) that are closest to them in terms of representation. Note, however, that other methods, e.g., averaging the distances to set $D$, or taking the median preference in $D$ would give perfectly valid but different scenarios of representation. 
Proposition 2.2. If a rule $\varphi$ is strategy-proof and ballot neutral, then it is also coalitional strategy-proof.

Proof. Let $\varphi$ be a strategy-proof and ballot neutral rule. For any $A \subsetneq \mathcal{A}$, any $N \subsetneq \mathcal{N}$ and any $P \in \mathcal{A}^{n}$, and for any $S \subseteq\{i \in N \mid P(i) \notin \varphi(P)\}$, let us denote any deviation from $P$ by agents in $S$ as $P^{\prime}=\left(P^{\prime}(S), P(N \backslash S)\right)$. Let $W=\varphi(P)$ and $O=\mathcal{L}(A) \backslash \varphi(P)$ denote a partition of $\mathcal{L}(A)$.

By Remark 2.1, there exists an expansion of $P$ by $\pi$, say $\bar{P}$, where $\bar{W}=\pi(W)$ and $\bar{O}=\pi(O)$ such that

$$
\max _{\bar{R}, R^{\prime} \in \bar{O}} \delta\left(\bar{R}, R^{\prime}\right)<\min _{\bar{R} \in \bar{O}, R^{\prime} \in \bar{W}} \delta\left(\bar{R}, R^{\prime}\right) .
$$

Consider any enumeration of $i \in S$, i.e., $S=\{1,2, \ldots, s\}$. Let us construct expanded profiles, $\bar{P}_{0}, \bar{P}_{1}, \ldots, \bar{P}_{s}$, with $\bar{P}_{0}=\bar{P}, \bar{P}_{s}=\bar{P}^{\prime}$ (the expansion of $P^{\prime}$ by $\pi$, i.e., $\bar{P}^{\prime}=\left(\bar{P}^{\prime}(S), \bar{P}(N \backslash S)\right)$, and for all $i \in\{1,2, \ldots, s\}, \bar{P}_{i}=$ $\left(\bar{P}^{\prime}(\{1,2, \ldots, i\}), \bar{P}(N \backslash\{1,2, \ldots, i\})\right)$. This is a formalization of the idea that any deviation by a coalition can be constructed as a result of consecutive unilateral deviations by a sequence of agents.

By Proposition 2.1, $\varphi\left(\bar{P}_{i}\right) \subseteq R P\left(\bar{P}_{i}\right)$ for all $i \in\{1,2, \ldots, s\}$. Note that from $\bar{P}_{0}$ to $\bar{P}_{1}$, there cannot be a preference $\bar{R} \in \bar{O}$ that becomes a new delegate for $\bar{P}_{1}$. This is because by (2.1), we have that for all $R^{\prime} \in \bar{W}, \delta(\bar{R}, \bar{P}(1))<\delta\left(R^{\prime}, \bar{P}(1)\right)$ and this would contradict individual strategy-proofness. A similar argument holds from $\bar{P}_{i}$ to $\bar{P}_{i+1}$ for any $i \in\{1,2, \ldots, s-1\}$. As the choice of enumeration of agents in $S$ is arbitrary, eventually this implies that there exists no $\bar{R} \in \bar{O}$ such that $\bar{R} \in \varphi\left(\bar{P}_{s}\right)$. As $\bar{P}_{s}=\bar{P}^{\prime}$, and $\bar{P}^{\prime}$ is an expansion of $P^{\prime}$, then there exists no $R \in O$, such that $R \in \varphi\left(P^{\prime}\right)$, since there exists no $\bar{R} \in O$ with $\bar{R} \in \varphi\left(\bar{P}^{\prime}\right)$. Then $\varphi\left(P^{\prime}\right) \subseteq W=\varphi(P)$. As $\varphi\left(P^{\prime}\right)$ is a subset of $\varphi(P)$, this implies that no agent in $S$ has become strictly better off, i.e.,

There exists no $i \in S$ such that $\min _{R \in \varphi(P)} \delta(P(i), R)>\min _{R \in \varphi\left(P^{\prime}\right)} \delta(P(i), R)$.

Since all agents whose preferences are already included in the delegation, i.e, $P(i) \in W$ has distance of zero to the delegation, they will not have any incentive to deviate or to join a coalition. This means that there cannot be any coalition $S$ which can successfully manipulate. 


\subsection{Using thresholds for delegation rules}

In this section, we introduce a large class of delegation rules which we call threshold rules. Every threshold rule is associated with a particular threshold function which we introduce below. Thereafter we show that the rules are well-defined and provide some examples within this special class of delegation rules.

Definition 2.6. Threshold Function: A threshold function is a function $f: \mathbb{Z}^{++} \rightarrow\left(\frac{1}{2}, 1\right] \cap \mathbb{Q}$ such that for all $t:$

$$
f(t) \geq \frac{f(t-1)+1}{2} .
$$

These functions simply assign a threshold for each possible delegation of size $t$. Let us introduce some additional notation to define the threshold rules. Given any $P \in \mathcal{L}(A)^{n}$, consider an enumeration which orders preferences according to their support from the agents from the strongest to weakest, i.e., $p_{i} \geq p_{i+1}$. For example, $p=(0.5,0 . \overline{3}, 0.1 \overline{6}, 0,0, \ldots, 0)$ denotes the normalized support for $P$. Let us also denote the corresponding preferences as $R_{1}, R_{2}, \ldots, R_{|A| \text { ! ,i.e., }}$ $R_{1}$ is the preference with the strongest support and so forth. ${ }^{14}$ Then we can define the cumulative support $\rho$ as the cumulative vector of $p$, i.e., for all $i$, $\rho_{i}=p_{1}+\ldots+p_{i}$. For instance, the cumulative support for the aforementioned $P$ is: $\rho=(0.5,0.8 \overline{3}, 1,1, \ldots, 1)$.

We first introduce the threshold rules as an algorithm, then proceed with the formal definition.

Take any profile $P$ and the cumulative support for it as $\rho$. Consider any threshold function $f$. Let $R_{1}, R_{2}, \ldots R_{|A| \text { ! denote an ordering of }}$ preferences according to their support, with ties broken arbitrarily.

Step 1: Check whether $\rho_{1} \geq f(1)$. If yes, $\varphi^{f}(P)=\left\{R_{1}\right\}$ and the algorithm stops. Otherwise, go to the next step.

\footnotetext{
${ }^{14}$ Note that some preferences in profiles might have equal support with a tie. In that case, the enumeration of those preferences can be chosen arbitrarily.
} 
Step 2: Check whether $\rho_{2} \geq f(2)$. If yes, $\varphi^{f}(P)=\left\{R_{1}, R_{2}\right\}$ and the algorithm stops. Otherwise, go to the next step.

Step t: Check whether $\rho_{t} \geq f(t)$. If yes, $\varphi^{f}(P)=\left\{R_{1}, R_{2}, R_{3}, \ldots, R_{t}\right\}$ and the algorithm stops. Otherwise, go to the next step.

Note that the algorithm stops after finite steps since we are dealing with a finite subset $A$ of $\mathcal{A}$. Next, we propose the formal definition. Again, given any profile $P$, we use the enumeration $R_{1}, R_{2}, \ldots R_{|A| \text { ! which }}$ orders according to the size of the support.

Definition 2.7. Threshold Rule: Given a threshold function $f$ and $P \in$ $\mathcal{L}(A)^{n}$, the threshold rule corresponding to $f$ is $\varphi^{f}(P)=\left\{R_{1}, R_{2}, \ldots, R_{t^{*}}\right\}$ where $t^{*}=\underset{t}{\arg \min }\left\{t \in \mathbb{Z}^{+} \mid \rho_{t} \geq f(t)\right\}$.

Thus the threshold rule select the lowest number of delegates at which the relevant threshold for total support is reached. However, there are two immediate concerns about these delegations rules. The first is whether we can always find a delegation that exceeds the threshold. The second is what happens when the algorithm stops at $t^{*}$, where two preferences have equal support, i.e., $p_{t^{*}}=p_{t^{*}+1}$ and $R_{t^{*}} \in \varphi^{f}(P)$ but $R_{t^{*}+1} \notin \varphi^{f}(P)$. We address both concerns in Proposition 2.3 which shows that the rules are well-defined.

Proposition 2.3. For all $A \subsetneq \mathcal{A}, N \subsetneq \mathcal{N}, P \in \mathcal{L}(A)^{n}$, and all threshold functions $f$, the threshold rule $\varphi^{f}(P)$ is well-defined.

Proof. The proof is in Appendix A.2.2.

Example 2.1. We will show four different threshold rules in the case of three alternatives. Let us consider the three different preference profiles, $P^{1}, P^{2}$, 
and $P^{3}$ denoted below by the normalized support (on the left side) and the cumulative support (on the right side) for preferences. Note that for the sake of simplicity we use profiles with the same enumeration wherein the support for $R_{i}$ is decreasing in $i$.

$$
\begin{array}{ll}
p^{1}=(0.31,0.29,0.29,0.11,0,0) & \rho^{1}=(0.31,0.6,0.89,1,1,1) \\
p^{2}=(0.78,0.12,0.1,0,0,0) & \rho^{2}=(0.78,0.9,1,1,1,1) \\
p^{3}=(0.55,0.12,0.11,0.11,0.11,0) & \rho^{3}=(0.55,0.67,0.78,0.89,1,1)
\end{array}
$$

The illustrations which is provided below capture the essence of threshold rules. Even though neither cumulative supports nor the relevant thresholds for each cardinality are continuous values, connecting discrete values via lines makes visualization easier. In the following graphs, the first number of delegates that a cumulative support is above the corresponding threshold indicates the number of delegates in the delegation.

Throughout these examples, let us denote the threshold function as $f=$ $(., \ldots, 1, \ldots)$, where the $i^{\text {th }}$ entry corresponds to $f(i)$. Since this function is increasing and has the bound of 1 , once the value of 1 is reached, all further values are equal to 1.

- The first rule, $\varphi^{1}$ is defined by the threshold function $f^{1}=$ $(0.51,0.76,0.89,0.95,1, \ldots)$. This rule checks whether the total support for some delegation reaches the relevant threshold for the size of the delegation, and if it does, picks that delegation with the smallest number of delegates.

- The second rule we deal with, $\varphi^{2}$ is characterized by the threshold vector, $f^{2}=(0.51,1, \ldots)$. This rule checks whether there exists any preference that is supported by more than at least $51 \%$ of the agents and if it is the case makes it the singleton delegate. If it is not the case, the rule picks all reported preferences instead.

- The third rule we deal with, $\varphi^{3}$ is characterized by the threshold vector, $f^{3}=(0 . \overline{6}, 1, \ldots)$. This rule checks whether there exists any preference that is supported by more than at least two-thirds of the agents (a.k.a. qualified 
majority), and if it is the case, then makes it the singleton delegate. If it is not the case, the rule picks all reported preferences instead.

- The last rule we deal with, $\varphi^{4}$ is the reported preference rule $R P(P)$, which chooses all preferences reported. The relevant threshold vector is $f^{4}=(1, \ldots)$, i.e., the total support for any delegation should be at least $100 \%$.

Illustrations of the rules and the delegations for each example profile are provided below. The bold numbers for $\rho_{i}$, indicate that $R_{i}$ is a chosen delegate for the profile $p$ under the rule $\varphi^{f}$.

$$
\begin{aligned}
& f^{1}=(0.51,0.76,0.89,0.95,1, \ldots) \\
& \rho^{1}=(\mathbf{0 . 3 1}, \mathbf{0 . 6}, \mathbf{0 . 8 9}, 1,1,1) \\
& \rho^{2}=(\mathbf{0 . 7 8}, 0.9,1,1,1,1) \\
& \rho^{3}=(\mathbf{0 . 5 5}, 0.67,0.78,0.89,1,1)
\end{aligned}
$$

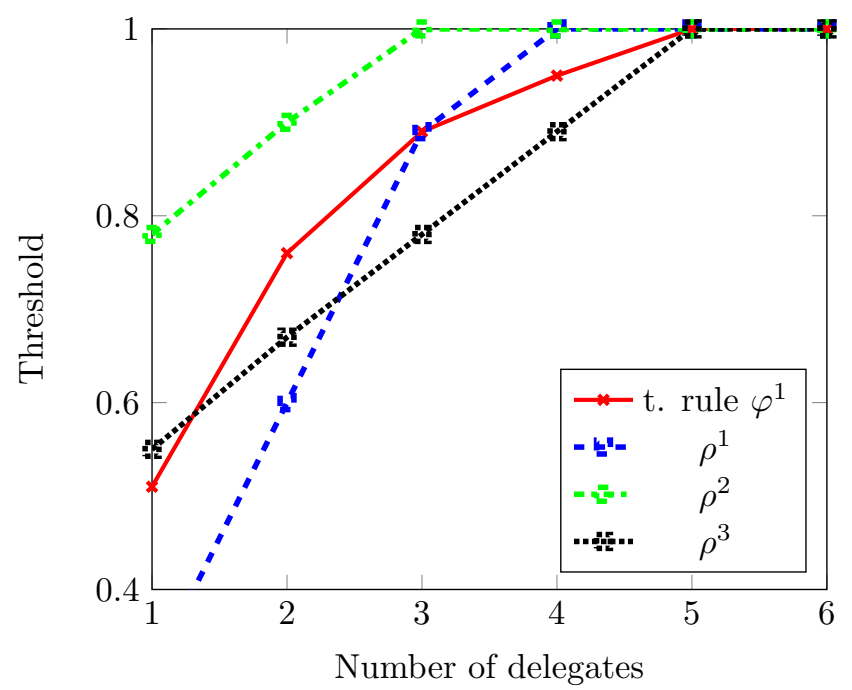

As it can be seen from the graph, $\varphi^{1}\left(P^{1}\right)=\left\{R_{1}, R_{2}, R_{3}\right\}$, while $\varphi^{1}\left(P^{2}\right)=$ 
$\varphi^{1}\left(P^{3}\right)=\left\{R_{1}\right\}$

$$
\begin{aligned}
f^{2} & =(0.51,1, \ldots) \\
\rho^{1} & =(\mathbf{0 . 3 1}, \mathbf{0 . 6}, \mathbf{0 . 8 9}, \mathbf{1}, 1,1) \\
\rho^{2} & =(\mathbf{0 . 7 8}, 0.9,1,1,1,1) \\
\rho^{3} & =(\mathbf{0 . 5 5}, 0.67,0.78,0.89,1,1)
\end{aligned}
$$

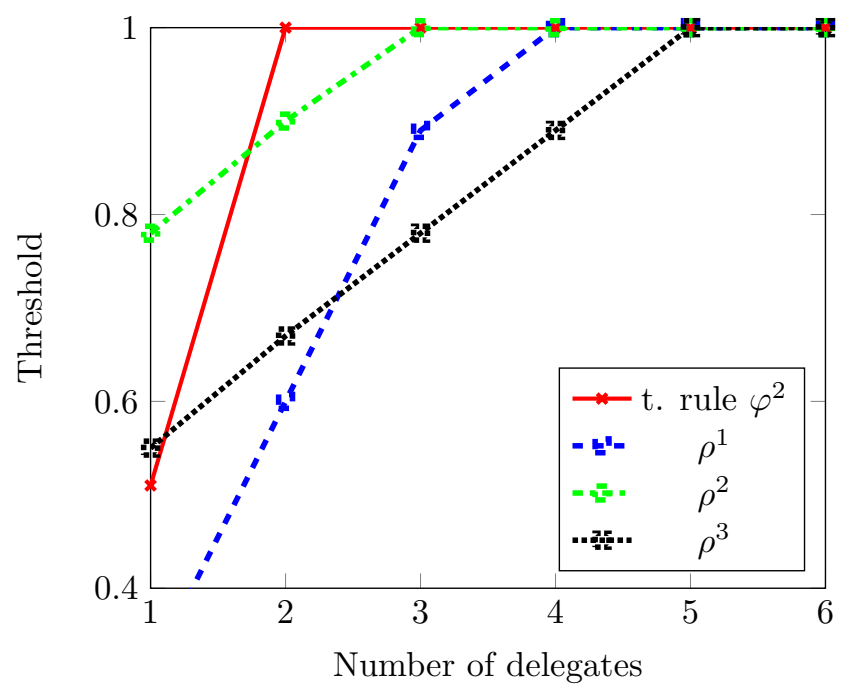

As it can be seen from the graph, $\varphi^{2}\left(P^{1}\right)=\left\{R_{1}, R_{2}, R_{3}, R_{4}\right\}$, while $\varphi^{2}\left(P^{2}\right)=\varphi^{2}\left(P^{3}\right)=\left\{R_{1}\right\}$. 


$$
\begin{aligned}
f^{3} & =(0 . \overline{6}, 1, \ldots) \\
\rho^{1} & =(\mathbf{0 . 3 1}, \mathbf{0 . 6}, \mathbf{0 . 8 9}, \mathbf{1}, 1,1) \\
\rho^{2} & =(\mathbf{0 . 7 8}, 0.9,1,1,1,1) \\
\rho^{3} & =(\mathbf{0 . 5 5}, \mathbf{0 . 6 7}, \mathbf{0 . 7 8}, \mathbf{0 . 8 9}, \mathbf{1}, 1)
\end{aligned}
$$

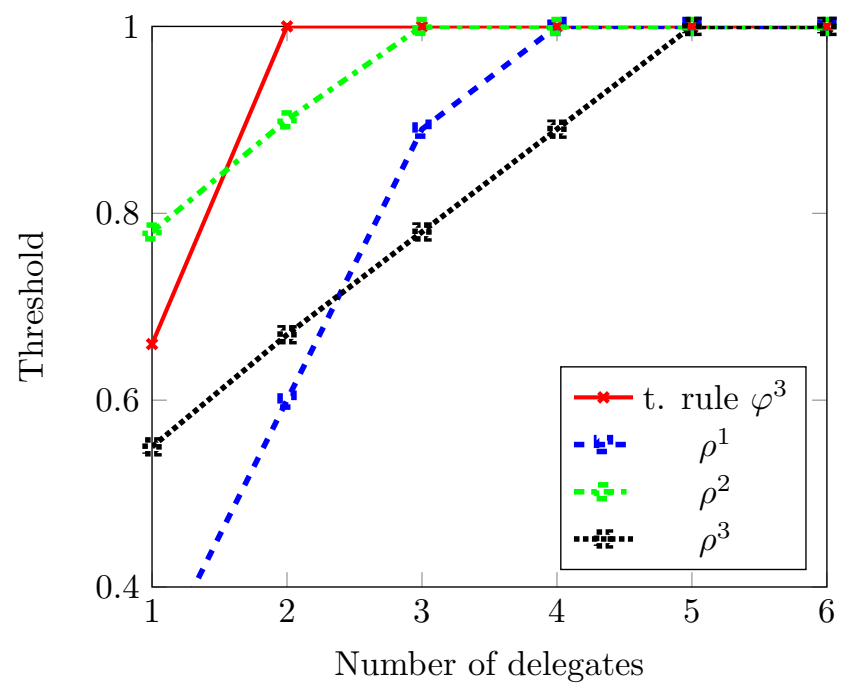

As it can be seen from the graph, $\varphi^{3}\left(P^{1}\right)=\left\{R_{1}, R_{2}, R_{3}, R_{4}\right\}, \varphi^{3}\left(P^{2}\right)=$ $\left\{R_{1}\right\}$, and $\varphi^{3}\left(P^{3}\right)=\left\{R_{1}, R_{2}, R_{3}, R_{4}, R_{5}\right\}$. 


$$
\begin{aligned}
f^{4} & =(1, \ldots) \\
\rho^{1} & =(\mathbf{0 . 3 1}, 0.6,0.89,1,1,1) \\
\rho^{2} & =(\mathbf{0 . 7 8}, 0.9,1,1,1,1) \\
\rho^{3} & =(\mathbf{0 . 5 5}, 0.67,0.78,0.89,1,1)
\end{aligned}
$$

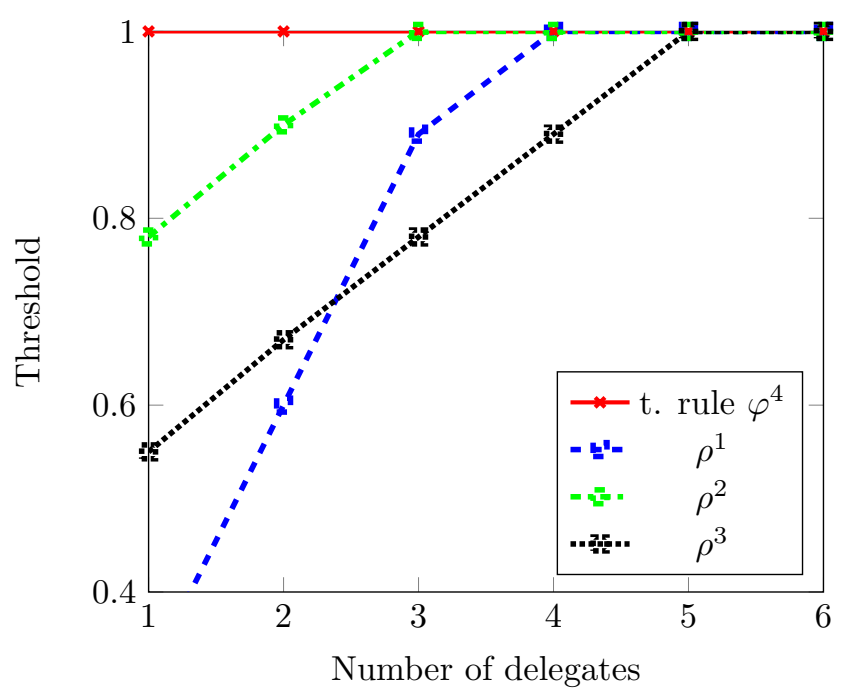

As it can be seen from the graph, $\varphi^{4}\left(P^{1}\right)=\left\{R_{1}, R_{2}, R_{3}, R_{4}\right\}, \varphi^{4}\left(P^{2}\right)=$ $\left\{R_{1}, R_{2}, R_{3}\right\}$, and $\varphi^{4}\left(P^{3}\right)=\left\{R_{1}, R_{2}, R_{3}, R_{4}, R_{5}\right\}$.

\subsection{Characterization of the threshold delega- tion rules}

In this section, we show that the conditions of Pareto optimality, consistency, ballot neutrality, and strategy-proofness characterize the class of delegation rules which we explained in the previous section. We show that these conditions lead to some implications concerning the behavior of the delegation rules. The first four lemmas shape the structure of the rules concerning the support of 
delegates. Another four lemmas prove the existence of a series of critical thresholds for choosing delegates and set forth the structure of these thresholds. We conclude the section with our main theorem which states that the only rules satisfying the conditions we demand are the threshold delegation rules.

\subsubsection{Delegates and their support in the society}

In what follows, Lemma 2.1 shows that if a preference is chosen as a delegate, then any other preference with stronger support in the society should also be chosen. Lemma 2.2 argues that rules should only care about the percentage of the support, i.e., only the normalized support of preference profiles matter. Lemma 2.3 proves that i) equal redistribution of the total support for the delegates among themselves does not change the delegation, and ii) equal redistribution of the total support for the rest among themselves also does not modify the delegation.

Lemma 2.1. If a rule $\varphi$ satisfies consistency, ballot neutrality, and strategyproofness, then for all $A \subsetneq \mathcal{A}, N \subsetneq \mathcal{N}$ and $P \in \mathcal{L}(A)^{n}$ if $R \in \varphi(P)$ and $p\left(R^{\prime}\right) \geq p(R)$, we have $R^{\prime} \in \varphi(P)$.

Proof. The proof is in Appendix A.2.3.

This lemma and Proposition 2.1 implies that any rule satisfying these conditions will have a delegation composed of preferences with relatively higher support compared to preferences that are not in the delegation. The following lemma proves the delegations to be the same for two different societies with identical normalized supports. Let $p / n=\left(\frac{p_{1}}{n}, \frac{p_{2}}{n}, \ldots, \frac{p_{|A| !}}{n}\right)$ denote the normalized support.

Lemma 2.2. If a rule $\varphi$ satisfies consistency and ballot neutrality, then for all $A \subsetneq \mathcal{A}, N, N^{\prime} \subsetneq \mathcal{N}$ and for all $P \in \mathcal{L}(A)^{n}, P^{\prime} \in \mathcal{L}(A)^{n^{\prime}}$ such that $p / n=p^{\prime} / n^{\prime}$, we have $\varphi(P)=\varphi\left(P^{\prime}\right)$.

Proof. The proof is in Appendix A.2.4.

The following lemma proves that neither averaging between supports of chosen delegates nor averaging between supports of non-delegates will change the delegation. 
Lemma 2.3. If a rule $\varphi$ satisfies consistency and ballot neutrality, then for all $A \subsetneq \mathcal{A}, N \subsetneq \mathcal{N}$ and $P \in \mathcal{L}(A)^{n}$, denoting $|\varphi(P)|=t$, and picking an enumeration on $\mathcal{L}(A)$ such that $p_{i} \geq p_{j}$ for all $i<j$, the following holds:

i) For any $P^{\prime} \in \mathcal{L}(A)^{n}$ such that $\frac{p_{j}^{\prime}}{n}=\sum_{i=1}^{t} \frac{p_{i}}{n t}$ for all $j \in\{1,2, \ldots, t\}$ and $\frac{p_{j}^{\prime}}{n}=\frac{p_{j}}{n}$ for all $j \in\{t+1, t+2, \ldots,|A| !\}$ we have $\varphi(P)=\varphi\left(P^{\prime}\right)$.

ii) For any $P^{\prime \prime} \in \mathcal{L}(A)^{n}$ such that $\frac{p_{j}^{\prime \prime}}{n}=\frac{p_{j}}{n}$ for all $j \in\{1,2, \ldots, t\}$ and $\frac{p_{j}^{\prime \prime}}{n}=$ $\sum_{i=t+1}^{|A| !} \frac{p_{j}}{n(|A| !-t)}$ for all $j \in\{t+1, t+2, \ldots,|A| !\}$ we have $\varphi(P)=\varphi\left(P^{\prime \prime}\right)$.

Proof. The proof is in Appendix A.2.5.

Example 2.2. As an example for those two cases, let us take some $A$ with $|A|=3$. Let us take $P \in \mathcal{L}(A)^{n}$ with support $p=(\mathbf{8}, \mathbf{7}, \mathbf{6}, 3,0,0)$ where bold numbers indicate the support for the chosen delegates. As an example for two subcases of the Lemma 2.3, let us take $P^{\prime}, P^{\prime \prime} \in \mathcal{L}(A)^{n}$ with supports $p^{\prime}=(7,7,7,3,0,0), p^{\prime \prime}=(8,7,6,1,1,1)$ respectively. Then, Lemma 2.3 implies $\varphi(P)=\varphi\left(P^{\prime}\right)=\varphi\left(P^{\prime \prime}\right)=\left\{R_{1}, R_{2}, R_{3}\right\}$.

Remark 2.3. Using permutations and merging as in the proof of Lemma 2.3, it is straightforward to see that the lemma also applies to any subset of delegates or non-delegates. That is, averaging between supports of some subset of chosen delegates or some subset of non-delegates will not change the delegation.

In the next lemma, we show that if a preference is chosen as a delegate, it must have more support than the total support for all the preferences which are not in the delegation. This is mainly due to the strategy-proofness condition.

Lemma 2.4. If a rule $\varphi$ satisfies ballot neutrality and strategy-proofness, then for all $A \subsetneq \mathcal{A}, N \subsetneq \mathcal{N}$, and $P \in \mathcal{L}(A)^{n}$ if $R \in \varphi(P)$, then we have

$$
p(R)>\sum_{R^{\prime} \notin \varphi(P)} p\left(R^{\prime}\right) .
$$

Proof. The proof is in Appendix A.2.6. 


\subsubsection{When to choose a delegate, and when not to?}

As seen in the previous four lemmas, any well-defined delegation rule satisfying the conditions of Pareto optimality, consistency, ballot neutrality, and strategyproofness takes the most supported preference in the delegation. However, for this preference to be the only delegate, it has to be powerful enough to eliminate all the other opinions. We need a new tool to capture this. Take any $\varphi$ which satisfies all the conditions. Categorize all $P \in \mathcal{L}(A)^{n}$ for any $N \subsetneq \mathcal{N}$ and $A \subsetneq \mathcal{A}$ according to the size of the delegations as follows: $\mathcal{P}_{t}=\left\{P \in \mathcal{L}(A)^{n}|| \varphi(P) \mid=t\right\}$. Lemma 2.1 and 2.2 imply that we only have to focus on the normalized support of the profiles from stronger to the weaker. Therefore, we can define a vector for this $\varphi$ for any $A \subsetneq \mathcal{A}$ as

$$
k^{\varphi}(A)=\left[k_{1}, k_{2}, \ldots, k_{|A| !}\right], \text { where each } k_{t}=\min _{P \in \mathcal{P}_{t}}\left(\sum_{i=1}^{t} p_{i}\right) / n .
$$

To ease the notation, we will omit $\varphi$ from $k^{\varphi}$ whenever it is clear. Furthermore, by ballot neutrality, we know that for these rules the vector $k$ is the same for every $A$ with equal cardinality. To understand these vectors, consider all profiles which end up with a single delegate under $\varphi$. Then $k_{1}$ gives us the relative support of the delegate with minimal value, among all the profiles with a single delegation. Similarly, $k_{i}$ gives the total relative support of the delegation with the minimal value, among all the profiles with a delegation of size $i$. In what follows, we discuss some features of these vectors.

Lemma 2.5 shows how $k_{i}$ values relate to one another. Lemma 2.6 shows if a preference has more relative support than $k_{1}$ it has to be chosen uniquely. Lemma 2.7 shows how the choice of delegates depends on $k$ in general. Finally, Lemma 2.8 shows how the vectors for sets of alternatives of different sizes relate to each other.

Lemma 2.5. If a rule $\varphi$ satisfies Pareto optimality, consistency, ballot neutrality, and strategy-proofness, then for all $A \subsetneq \mathcal{A}$, and $N \subsetneq \mathcal{N}$ the corresponding vector satisfies that $k_{t}^{\varphi}(A) \geq \frac{k_{t-1}^{\varphi}(A)+1}{2}$ for all $t \in\{2,3, \ldots,|A| !\}$.

Proof. The proof is in Appendix A.2.7. 
In the following two lemmas, Lemma 2.6 and 2.7, we assume an enumeration which orders preferences according to their support from the agents from the strongest to weakest, i.e., $p_{i} \geq p_{j}$ for all $i<j$. Lemma 2.6 shows that for profiles in which a strongest single preference has a relative support $p_{1} / n$ more than $k_{1}$, the delegation should only consist of this preference, $R_{1}$. Lemma 2.7 extends this to larger delegation sizes, i.e., the delegation should comprise of the first $t$ strongest preferences whose relative total support surpasses their corresponding threshold $k_{t}$ while no smaller subdelegation satisfies this.

Lemma 2.6. If a rule $\varphi$ satisfies Pareto optimality, consistency, ballot neutrality, and strategy-proofness, then for all $A \subsetneq \mathcal{A}, N \subsetneq \mathcal{N}$ and for any $P \in \mathcal{L}(A)^{n}$ such that $p_{1} \geq n k_{1}^{\varphi}(A)$, we have that $\varphi(P)=\left\{R_{1}\right\}$.

Proof. The proof is in Appendix A.2.8.

Lemma 2.7. If a rule $\varphi$ satisfies Pareto optimality, consistency, ballot neutrality, and strategy-proofness, then for all $A \subsetneq \mathcal{A}, N \subsetneq \mathcal{N}$ and for any $P \in \mathcal{L}(A)^{n}$ such that

i) for some $t>1, \sum_{i=1}^{t} p_{i} \geq n k_{t}^{\varphi}(A)$ and,

ii) for all $l<t, \sum_{i=1}^{l} p_{i}<n k_{l}^{\varphi}(A)$

we have: $\varphi(P)=\left\{R_{1}, R_{2}, \ldots, R_{t}\right\}$.

Proof. The proof is in Appendix A.2.9.

The next lemma shows that the corresponding vectors of the rules are independent of the number of alternatives.

Lemma 2.8. If a rule $\varphi$ satisfies ballot neutrality, then for any $A \subsetneq \bar{A} \subsetneq \mathcal{A}$, the corresponding vector satisfies that $k^{\varphi}(A)_{t}=k^{\varphi}(\bar{A})_{t}$ for all $t \in\{1,2, \ldots,|A| !\}$.

Proof. The proof is in Appendix A.2.10.

Note that Lemma 2.8 has further implications. In fact, for any two sets of alternatives, $A, B$ the $k^{\varphi}(A)_{t}$ and $k^{\varphi}(B)_{t}$ values will always correspond to each other. That can be achieved by extending each of the sets to $A \cup B$ by separately by implementing the lemma above. 
Next, our main theorem finalizes the result by showing there is only one class of delegation rules, i.e., the threshold rules, associated with a threshold function that satisfies all the conditions we have imposed.

Theorem 2.1. A rule $\varphi$ satisfies Pareto optimality, consistency, ballot neutrality, and strategy-proofness if and only if for all $A \subsetneq \mathcal{A}, N \subsetneq \mathcal{N}$ and $P \in \mathcal{L}(A)^{n}$ we have that $\varphi(P)=\varphi^{f}(P)$ for some threshold function $f$.

Proof. We leave the if part to the reader and prove the only if part. Proposition 2.1 and Lemma 2.1 together imply that only preferences with higher support will be assigned as delegates as opposed to those with lower support. Lemma 2.2 implies that the degree of this relative power will be independent of the number of agents. Lemma 2.3 states that averaging of supports for delegates will not change the delegation. This implies that only the normalized supports for the delegations matter.

Given any rule $\varphi$ satisfying the conditions, we can then construct a corresponding vector $k(A)$ where the $i^{\text {th }}$ entry is the minimum support needed for a delegation of size $i$ across all preference profiles on $A$. By consistency, $k$ is constant across all possible subsets of agents $N \subsetneq \mathcal{N}$, and by Lemma 2.8, $k(A)_{i}=k(B)_{i}$, i.e., $k$ is also constant across all possible sets of alternatives $A \subsetneq \mathcal{A}$. Hence, regardless of the domain of the delegation rule, one can construct a function $f$ on positive integers such that $f(i)=k_{i}$ for all possible delegations of size $i$. By Lemma 2.4, we have that $f(1)>1 / 2$. By Lemma 2.5, for all $t>1$ we have that

$$
f(t) \geq \frac{f(t-1)+1}{2}
$$

Thus, by construction, $f: \mathbb{Z}^{+} \rightarrow\left(\frac{1}{2}, 1\right] \cap \mathbb{Q}$ is the unique threshold function induced by $\varphi$ as in Definition 2.6, and hence $\varphi=\varphi^{f}$ is a threshold delegation rule.

\subsection{Conclusion}

This chapter brings about a novel class of rules for choosing a delegation, characterized by intuitive fairness, efficiency, and non-manipulability proper- 
ties. The nature of these threshold delegation rules is such that they provide a good compromise in at least three aspects, inclusivity, minimalism, and non-manipulability. Inclusivity is often deemed as crucial since it results in the legitimacy of the political settlement (Dudouet and Lundström (2016)). Minimalism, in the sense that not everyone can be invited to the table, is an important parameter of simplicity of design in conflict resolution. Finally, non-manipulability of a delegation rule is essential so that people's true opinions are always reflected in the conflict resolution, preventing further re-escalation of post-truce conflicts.

Let $t$ denote the size of the delegation, and $f$ denote a threshold function. Then all the threshold functions obey the following lower bounds:

$$
f(t)>\frac{1}{2}+\left(\frac{1}{2}\right)^{2}+\ldots+\left(\frac{1}{2}\right)^{t}=\sum_{i=1}^{t}\left(\frac{1}{2}\right)^{i}
$$

On top of this common lower bound feature, each of the threshold rules we characterize differs in the amount of representation they require from a delegation. For instance, if a single delegate is sent by the rule to the table, then that delegate, and the opinion she represents must have strictly more than $50 \%$ support in the society. However, let us consider a more demanding threshold rule, such as one that requires a $60 \%$ for a single delegate representation. In that case, the minimal required support for a delegation of size 2 becomes "at least" $80 \%$ (averaging $60 \%$ and 100\%). The thresholds for larger delegations quickly increase by averaging each threshold with $100 \%$ to find the next threshold, making it harder for small delegations to reach. Therefore, these rules are fairly inclusive in most of the cases. Essentially, the size of the delegations under the threshold rules depend on the diversity and the modality of the preferences in the society.

There are directions that we foresee for future research concerning the delegation choice. For instance, our selection of minimal Kemeny distance as a measure of representation of agents leads to a particular definition of strategy-proofness, which implies non-externality in representation. That is, we define a manipulation to be beneficial for an individual when the individual can successfully alter the "minimal distance" to the delegation. This interpretation 
implies that agents do not benefit or get harmed by other farther opinions in the delegation. Of course, one might define strategy-proofness as the inability of individuals to manipulate the "average distance to the delegation" or "distance to the farthest delegate" instead. Both these interpretations include externality in representation, i.e., introducing additional delegates might reduce the representation of agents.

Another direction is the choice of metric used to define strategy-proofness. Although Kemeny distance is quite standard in the literature, one might employ other metrics to see the robustness of these findings. We would like to note, however, that none of our proofs uses the properties of Kemeny distance specifically. As long as the clustering result in Remark 2.1 can be reached, we expect that our results can be generalized. We suspect that any metric that satisfies a minimal set of common features/axioms ${ }^{15}$ will lead to similar results.

We conclude this chapter by suggesting the policymakers that when a peace delegation, a committee or a board of size $t$ is summoned from different opinions, then the ratio of uninvited opinions should be less than $(0.5)^{t}$ of the population, e.g., if only 3 delegates are invited, then uninvited groups should account for less than $12.5 \%$ of the population. This gives us a minimal bound for inclusivity, furthermore can help us to quantify "negligible minorities".

\footnotetext{
${ }^{15}$ We suspect one of this particular axioms to be the betweenness axiom introduced in Kemeny (1959).
} 


\section{Chapter 3}

\section{Condorcet versus \\ participation criterion in social welfare rules ${ }^{1}$}

\subsection{Introduction}

Two of the best known criteria for social choice rules are the Condorcet criterion and the participation criterion. The former, introduced by Marquis de Condorcet (1785), requires that if there is a candidate who beats all others in a pairwise comparison then it should be the unique winner. The rules that satisfy this property are also called Condorcet methods. The latter, introduced in Fishburn and Brams (1983) as No Show Paradox, requires that attending to an election should be somewhat incentive compatible. To be more precise, for any voter, participating in an election should not change the result into a less desirable outcome ${ }^{2}$ for that voter. Moulin (1988) shows that the Condorcet

\footnotetext{
${ }^{1}$ Based on Can, Ergin, and Pourpouneh (2017).

${ }^{2}$ For further results related to participation criteria or No Show Paradox, see Pérez (2001); Felsenthal and Nurmi (2016); Brandt, Geist, and Peters (2016); Núñez and Sanver (2017); Kasper, Peters, and Vermeulen (2017).
} 
criterion and the participation criterion are incompatible. ${ }^{3}$ To put it differently, there exists no Condorcet method which satisfies the participation criterion, hence they are all vulnerable to No Show Paradox.

In the more general setting which allows multiple winners in the outcome, Jimeno, Pérez, and García (2009) show that an extension of this incompatibility still persists if the agents are considered as optimistic or pessimistic, that is when comparing two sets of winners, they only consider the best or the worst one, respectively. Following this idea of sets as outcomes, we turn our attention to social welfare rules, i.e., rules that assign rankings of alternatives to each preference profile instead of a winning alternative. This chapter investigates if this same incompatibility extends to the framework of social welfare rules.

A natural extension of Condorcet criterion to social welfare rules is already defined in Young and Levenglick (1978), and this criterion is also used in this chapter. It implies that if an alternative is preferred to another by a majority of the population, the latter should not be put directly above the former in any of the rankings in the outcome. This criterion has two main advantages. First, as in the original paper, we also use a framework with multiple rankings in the outcome, and the criterion is suitable to use with such rules. Second, if we reduce a social welfare rule, which satisfies this criterion, to a social choice rule by selecting the top alternatives in each ranking, then this reduction will satisfy the Condorcet criterion.

To our knowledge, an analogous participation criterion in the framework for social welfare rules has not been defined. This chapter introduces a participation criterion which again deals with pairs of alternatives. Consider an agent who abstained from an election, but ranks any two pair of alternatives in the same way that a ranking which is part of the outcome. Then, our participation criterion requires that in the alternative case that this agent joins the election instead, every ranking in the outcome should rank those two pairs in the same way. If a social welfare rule which satisfies this criterion is reduced, the reduction, i.e., social choice rule that includes all topmost alternatives, will satisfy the participation criterion for social choice rules.

3 The incompatibility holds so long as there are at least four alternatives and twenty-five individuals. 
We show that these two criteria for social welfare rules are also incompatible. Then we consider two weaker versions for both criteria. To weaken the previous Condorcet criterion, we consider only cases where the relation implied by the majority is a total order. According to this weaker version, if the majority relation is a total order, only this total order should be selected as the outcome. Somewhat surprisingly, even this weaker condition is not enough to escape the impossibility if used together with the mentioned participation requirement. Next, we turn our attention to a weaker participation criterion which requires that no agent will be able to get a closer ranking in the outcome by abstaining than the closest one with his participation. Here, to define closeness we use the well-known Kemeny distance (Kemeny, 1959). ${ }^{4}$ Following this approach, we reach a possibility. We show that the Kemeny ${ }^{5}$ rule satisfies the Condorcet criterion (in Young and Levenglick (1978)) and the weaker participation criterion. Among the other rules we checked, there is no other rule that satisfies these two criteria together.

The rest of this chapter is organized as follows. In Section 3.2, we introduce the notation and the model. In Section 3.3, we define two pairs of Condorcet criteria and participation criteria for social welfare rules. In Section 3.4, we show logical implications between those criteria. Section 3.5 discusses various social welfare rules and checks which criteria is satisfied by each. Section 3.6, concludes the chapter with discussion and open questions.

\subsection{Model}

Let $N=\{1,2, \cdots, n\}$ be a non-empty and finite set of $n$ individuals and let $A$ be a non-empty and finite set of $m$ alternatives. Let $\mathcal{L}$ denote the set of all possible linear orders (strict rankings) over $A$, i.e., complete, reflexive, transitive, and antisymmetric binary relations over $A$. For a generic $R \in \mathcal{L}$ and any two alternatives $a, b \in A,(a, b) \in R$ is interpreted as " $a$ is at least as good as $b$ at $R$ ". In case $a$ and $b$ are two distinct alternatives the relation is

\footnotetext{
${ }^{4}$ See also Can and Storcken (2018) for a more recent characterization.

${ }^{5}$ Kemeny rule, also known as Kemeny-Young method, is introduced in Young and Levenglick (1978).
} 
strict. To simplify notation, we write $a b \in R$ instead of $(a, b) \in R$. Similarly $R=a b c d$ denotes a full ranking where $R=\{a a, a b, a c, a d, b b, b c, b d, c c, c d, d d\}$. To denote a ranking $R$ in which there is a pair $a b \in R$ whose elements are immediate successors, we use $R=\ldots a b \ldots$

A (preference) profile $p=(p(1), \cdots, p(n))$ is an element of in $\mathcal{L}^{N}$, where $p(i)$ is the preference ordering of individual $i$ over $A$. We denote rank of an alternative within a preference of an agent by $\operatorname{rank}(a, p(i))=\mid\{b \in A \mid b a \in$ $p(i)\} \mid{ }^{6}$ Given $p \in \mathcal{L}^{N}$, the profile of all individuals except $i$ is denoted by $p_{-i} \in \mathcal{L}^{N \backslash\{i\}}$, i.e., $p_{-i}=(p(1), \cdots, p(i-1), p(i+1), \cdots, p(n))$. We use $n_{a b}$ to denote the difference in numbers of people who prefer $a$ to $b$ and of those who prefer $b$ to $a$, i.e., $n_{a b}(p)=|\{i \in N \mid a b \in p(i)\}|-|\{i \in N \mid b a \in p(i)\}|$. We denote the corresponding tournament $T_{p}$ of a profile $p$ by $T_{p}=\{a b \in A \times A \mid$ $\left.n_{a b}(p)>0\right\}$. Moreover, we say $a$ beats $b$ whenever $a b \in T_{p}$.

We use this to define utilities of agents as: $u_{i}: A \rightarrow \mathbb{R}$ such that $u_{i}(a) \geq u_{i}(b)$ if and only if $a b \in p(i)$. Similarly, we denote the utility of an agent $i$ over the set of rankings by $U_{i}: \mathcal{L} \rightarrow \mathbb{R}$ such that $U_{i}(R) \geq U_{i}\left(R^{\prime}\right)$ if and only if $\delta(p(i), R) \leq \delta\left(p(i), R^{\prime}\right)$ where for any $R, R^{\prime} \in \mathcal{L}, \delta\left(R, R^{\prime}\right)=\left(\left|R \backslash R^{\prime}\right|+\left|R^{\prime} \backslash R\right|\right) / 2$, i.e., the Kemeny distance ${ }^{7}$ between $R$ and $R^{\prime}$. Note that, as $R$ and $R^{\prime}$ are both linear orders, we have, $\delta\left(R, R^{\prime}\right)=\left|R \backslash R^{\prime}\right|=\left|R^{\prime} \backslash R\right|=\left(m^{2}+m\right) / 2-\left|R \cap R^{\prime}\right|$.

\subsection{Extension of the Criteria to Social Welfare Rules}

We first remind the reader of the formal definitions of the Condorcet and the participation criteria on social choice rules. Thereafter we extend these concepts to social welfare rules.

\footnotetext{
${ }^{6}$ Because of reflexivity minimum rank is 1 .

${ }^{7}$ The Kemeny distance is introduced and characterized in Kemeny (1959), an improved characterization can be found in Can and Storcken (2018).
} 


\subsubsection{Social Choice Rules}

A social choice rule is a map $\psi: \mathcal{L}^{N} \rightarrow 2^{A} \backslash \emptyset$. For $p \in \mathcal{L}^{N}$, an alternative $a \in A$ is the Condorcet winner if $n_{a b}(p)>0$ for all $b \in A \backslash\{a\}$.

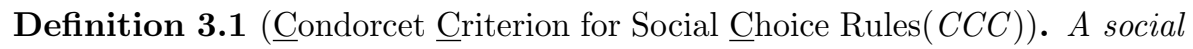
choice rule $\psi$ satisfies the Condorcet criterion for social choice rules whenever for all $p \in \mathcal{L}^{N}$, with a Condorcet winner $a \in A$, we have $\psi(p)=a$.

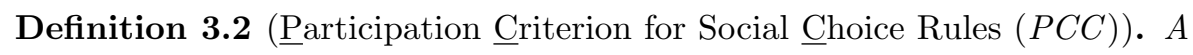
social choice rule $\psi$ satisfies participation criterion for social choice rules whenever for all $p \in \mathcal{L}^{N}$, and for all $i \in N$,

$$
\max _{a \in \psi(p)} u_{i}(a) \geq \max _{b \in \psi\left(p_{-i}\right)} u_{i}(b)
$$

That is, the best outcome of the social choice rule for each individual when he participates is at least as good as the best outcome when he does not participate. Note that, this definition corresponds to "ordering for optimists" defined in Jimeno, Pérez, and García (2009).

Remark 3.1 (Jimeno, Pérez, and García (2009)). There is no social choice rule satisfying participation criterion and the Condorcet criterion.

\subsubsection{Social Welfare Rules}

A social welfare rule $\varphi: \mathcal{L}^{N} \rightarrow 2^{\mathcal{L}} \backslash \emptyset$, is a correspondence that maps every preference profile $p$ to a non-empty subset of linear orders. Typically the Arrovian framework considers the range of social welfare rules to be weak orders i.e., the outcome might admit ties. Consider for instance the cyclical profile with three individuals and three alternatives, $p=(a b c, b c a, c a b)$. Then, any neutral Arrovian social welfare rule $\varphi$, will assign total indifference, $\varphi(p)=a \sim b \sim c$, where $\sim$ denotes the indifference. However, there are rules that aggregate preference profiles into multiple strict rankings without ties such as the Kemeny rule which would be introduced in Subsection 3.5.4. The Kemeny rule would assign the following three rankings as the outcome to the aforementioned profile, $\varphi^{\text {Kemeny }}(p)=\{a b c, b c a, c a b\}$. 
Note that, any weak order can be extended to a set of strict rankings, by simply breaking all ties neutrally. Therefore each Arrovian social welfare function can also be treated as a social welfare correspondence assigning possibly multiple strict rankings as the outcome. This gives comparability across all kinds of ranking aggregators. In what follows we shall consider social welfare rules that assign a set of strict rankings to each preference profile. In this context the outcome of the first rule in the example above will be all possible linear extensions of the weak order $\varphi(p)=a \sim b \sim c$, will be interpreted as, $\varphi(p)=\{a b c, a c b, b a c, b c a, c a b, c b a\}$.

\subsubsection{Strong Criteria on Social Welfare Rules}

First, we define a Condorcet criterion and a participation criterion for social welfare rules. These imply the criteria above for social choice rules if one considers a reasonable reduction of social welfare rules to social choice rules. The reduction operation, and implications are included in Section 3.4.

The following criterion is about immediate successors and requires two things. First, if an alternative, say $a$, is preferred to some other alternative, say $b$, by a majority of the population, $b$ can not be put immediately above $a$ in any of the rankings in the outcome. Second, if there is a pairwise tie between two alternatives, say $a$ and $b$, then a ranking can put $a$ immediately over $b$ only if there is another ranking in the outcome which puts $b$ immediately over $a$. Note that, this criteria does not imply anything when there are other alternatives between $a$ and $b$.

Definition 3.3 (Condorcet Criterion for Social Welfare Rules $(C C W)$ ). (Young and Levenglick (1978)) A social welfare rule $\varphi$ satisfies the Condorcet criterion for social welfare rules if for any $p \in \mathcal{L}^{N}$ and any distinct $a, b \in A$,

1. $n_{a b}(p)>0$ implies there exists no $R \in \varphi(p)$ such that $R=\cdots b a \cdots$.

2. $n_{a b}(p)=0$ implies for all $R \in \varphi(p)$ such that $R=\cdots a b \cdots$ there exists $R^{\prime} \in \varphi(p)$ such that $R^{\prime}=\cdots b a \cdots$.

The following criterion ensures that if an ordered pair in an individual preference is already in one ranking in the outcome during his absence, then 
that ordered pair should be in all rankings in the outcome after participation by this agent.

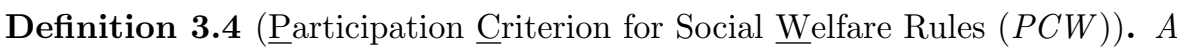
social welfare rule $\varphi$ satisfies participation criterion whenever for all $p \in \mathcal{L}^{N}$, and for all $i \in N$,

$$
\left(p(i) \cap \bigcup_{R \in \varphi\left(p_{-i}\right)} R\right) \subseteq \bigcap_{R \in \varphi(p)} R .
$$

That is if a is ranked over $b$ in some of the outcomes when $i$ is absent, and $i$ prefers a to $b$, then a should be ranked over $b$ in all of the outcomes when $i$ shows up.

The intuition behind this criterion is simple. For any ordered pair rankings in the outcome disagree upon, the participation of an agent forces the outcomes to agree on that pair according to the agent's preference. For cases in which every ranking in the outcome already agrees upon an ordered pair, participation by an agent who also agrees on the pair will not result in any ranking that disagrees to be included in the outcome.

\subsubsection{Weak Criteria on Social Welfare Rules}

Note that above, Condorcet Criterion is binding whenever there is a majority that puts some alternative over other. In the weaker Condorcet criterion, we check only the cases where all such pairs implies a total order over all elements of $A$. Formally, if we let $p \in \mathcal{L}^{N}$ be a profile, then a ranking $R \in \mathcal{L}$ is called the Condorcet ranking whenever $n_{a b}(p)>0$ for all $a b \in R$.

Definition 3.5 (Weak Condorcet Criterion for Social Welfare Rules $(W C C W))$. A social welfare rule $\varphi$ satisfies weak Condorcet criterion whenever for all $p \in \mathcal{L}^{N}$, with a Condorcet ranking $R \in \mathcal{L}$, we have $\varphi(p)=\{R\}$.

As the weaker participation criterion, we use comparison with respect to the Kemeny distance. According to the following criteria the Kemeny distance between ranking of an agent and the closest ranking in the outcome should weakly increase if the agent abstains. 


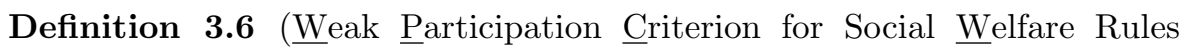
$(W P C W))$. A social welfare rule $\varphi$ satisfies weak participation criterion whenever for all $p \in \mathcal{L}^{N}$, and for all $i \in N$,

$$
\max _{R \in \varphi(p)} U_{i}(R) \geq \max _{R^{\prime} \in \varphi\left(p_{-i}\right)} U_{i}\left(R^{\prime}\right)
$$

That is, the best outcome of the social welfare rule for each individual when he participates is at least as good as the best outcome when he does not participate.

In the next section, we show the logical implications of the criteria we covered so far.

\subsection{Results}

To see whether the criteria we are interested in are actually informative in the framework of social choice rules, we need a way to reduce social welfare rules to social choice rules. The most natural way to do this would be defining the reduction operation so that, for any profile, the corresponding social choice rule will just pick the top alternatives in each ranking in the outcome of the social welfare rule.

Definition 3.7 (Corresponding Social Choice Rule). For a social welfare rule $\varphi$, the corresponding social choice rule $\psi^{\varphi}$ is defined by:

$$
a \in \psi^{\varphi}(p) \Leftrightarrow \text { there is } R \in \varphi(p) \text { such that } a b \in R \text { for all } b \in A \text {. }
$$

Our first proposition proves that the Condorcet criterion we use in social welfare rules implies the Condorcet criterion in social choice rules, assuming the reduction operation defined above.

Proposition 3.1. If $\varphi$ satisfies $C C W$, then the corresponding social choice rule $\psi^{\varphi}(p)$ satisfies $C C C$.

Proof. Note that, if there is a Condorcet alternative for some profile $p$, say $a, n_{a b}(p)>0$ for all $b \in A \backslash\{a\}$. A rule $\varphi$ which satisfies $C C W$, must place 
that $a$ at the top of all of rankings in the outcome. This means, corresponding social choice rule, $\psi^{\varphi}$ will only assign $a$ to the profile, that is $\psi^{\varphi}(p)=\{a\}$.

The second proposition shows that the analogue for the participation criteria in two different frameworks.

Proposition 3.2. If $\varphi$ satisfies $P C W$, then the corresponding social choice rule $\psi^{\varphi}(p)$ satisfies $P C C$.

Proof. Let us take some $\varphi$ which satisfies $P C W$. Suppose for a contradiction, corresponding social choice rule $\varphi, \psi^{\varphi}(p)$ does not satisfy PCC. This means, there exists $p \in \mathcal{L}(A)^{N}$, and $i \in N$ s.t.

$$
\max _{a \in \psi^{\varphi}\left(p_{-i}\right)} u_{i}(a)>\max _{b \in \psi^{\varphi}(p)} u_{i}(b) .
$$

Let us denote $x=\underset{a \in \psi^{\varphi}\left(p_{-i}\right)}{\arg \max } u_{i}$ and $y=\underset{b \in \psi^{\varphi}(p)}{\arg \max } u_{i}$ with $x \neq y$.

Note that $x y \in p(i)$. By definition of corresponding social choice rule $x$ should be the first entry in some ranking in $\varphi\left(p_{-i}\right)$. This means there exists a ranking in $R \in \varphi\left(p_{-i}\right)$ with $x y \in R$. Since $x y$ is also in $p(i), P C W$ requires $x y \in R^{\prime}$ for all $R^{\prime} \in \varphi(p)$, which means $y$ can not be at top of any ranking within $\varphi(p)$. This contradicts $y \in \psi^{\varphi}(p)$ in the first place.

Next, we show that the stronger criteria actually imply the weaker ones, as the naming suggests.

\section{Proposition 3.3. $C C W$ implies $W C C W$.}

Proof. Consider the case that there exists a Condorcet ranking for some profile $p$, with $R \in \mathcal{L}$ with $n_{a b}(p)>0$ for all $a b \in R$. Let us denote this ranking by $R=x_{1} x_{2} x_{3} \ldots x_{m}$. Take any $\varphi$ that satisfies $C C W$. Note that, $n_{x_{1} x_{k}}(p)>0$ would be true for all $k \in\{2, \cdots, m\}$. Then, by $C C W, x_{1}$ will be the top alternative in any ranking in the outcome, since otherwise we would have a $R^{\prime} \in \varphi(p)$ with $R^{\prime}=\cdots x_{l} x_{1} \cdots$ and $n_{x_{1} x_{l}}(p)>0$ for all $l \in\{2, \cdots, m\}$.

Similarly, $x_{2}$ will be at the secondary place all rankings in the outcome, since no alternative other than $x_{1}$ can be put directly on top of it by a similar reasoning. Iteratively, $R$ remains the only possible outcome, which will ensure $W C C W$. 
Proposition 3.4. $P C W$ implies $W P C W$.

Proof. Assume that $\varphi$ satisfies $P C W$. Take any preference profile $p \in$ $\mathcal{L}(A)^{N}$, and an agent $i \in N$. Let us denote $\bar{R}=\underset{R \in \varphi\left(p_{-i}\right)}{\arg \min } \delta(R, p(i))$ and $R^{\prime}=\underset{R \in \varphi(p)}{\arg \min } \delta(R, p(i))$. It is sufficient to show that

$$
\delta(p(i), \bar{R}) \geq \delta\left(p(i), R^{\prime}\right)
$$

Take any $a b \in \bar{R} \cap p(i)$. Since $a b \in p(i)$, from $P C W, a b \in R^{*}$ for all $R^{*} \in \varphi(p)$, including $R^{\prime}$. Which means for every pair in which $p(i)$ and $\bar{R}$ agrees, $R^{\prime}$ also agrees. Formally:

$$
(p(i) \cap \bar{R}) \subseteq\left(p(i) \cap R^{\prime}\right)
$$

This also means that:

$$
|p(i) \cap \bar{R}| \leq\left|p(i) \cap R^{\prime}\right|
$$

Since $\bar{R}, R^{\prime}$ and $p(i)$ are linear orders with cardinality $\left(m^{2}+m\right) / 2$, the following holds:

$$
\delta(\bar{R}, p(i))=\frac{m^{2}+m}{2}-|p(i) \cap \bar{R}| \geq \frac{m^{2}+m}{2}-\left|p(i) \cap R^{\prime}\right|=\delta\left(R^{\prime}, p(i)\right)
$$

which completes the proof.

Next, we show that $P C W$ and $W C C W$ are incompatible.

Proposition 3.5. There exists no social welfare rule which satisfies $P C W$ and $W C C W$.

Proof. The proof is in Appendix B.1.

Note that this incompatibility, together with Proposition 3.3 implies the following:

Corollary 3.1. There exists no social welfare rule which is $P C W$ and $C C W$. 
Proof. Proposition 3.5 and Proposition 3.3 together imply this.

We see that as long as we insist on the stronger definition for participation condition, even using a weaker version of the Condorcet criterion leads to an incompatibility between participation and Condorcet concepts. However, if participation criterion is weakened instead, a possibility result is reached. In Subsection 3.5.4, we show that Kemeny rule is an example that satisfies both $W P C W$ and $C C W$, although we suspect that there might be other rules in the same vein.

In Figure 3.1, we summarize the findings above. Next, we will check whether some well known rules satisfy the criteria we are interested in.

Figure 3.1: Logical relations

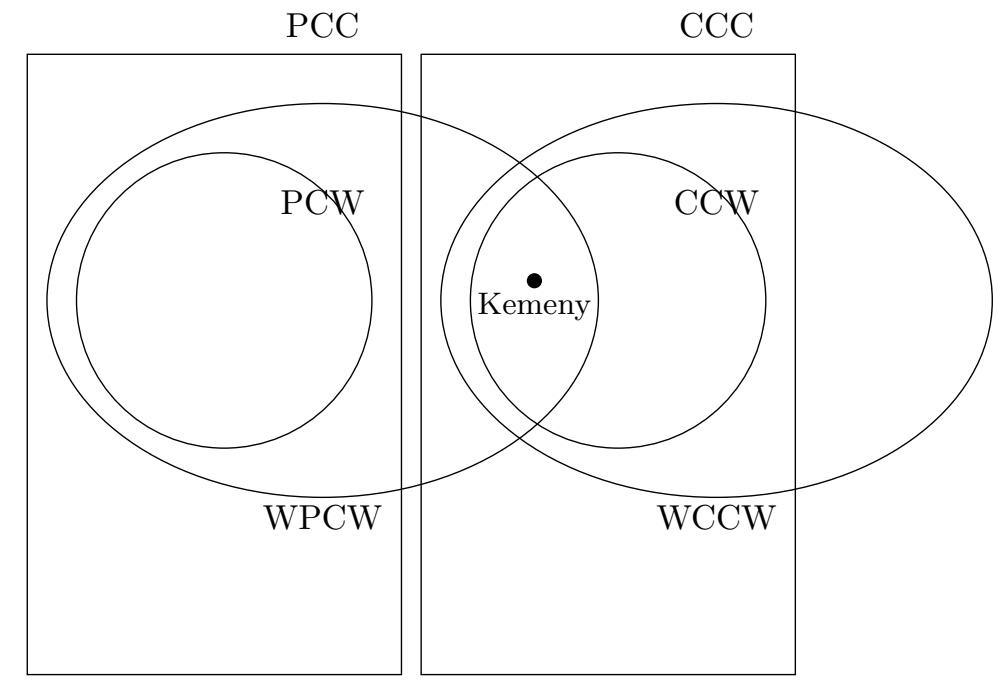

\subsection{Specific Rules}

In this section, we investigate the Copeland, Minimax, scoring, Kemeny and Slater welfare rules to see whether they satisfy the Condorcet ( $W C C W$ and $C C W$ ) and participation ( $W P C W$ and $P C W$ ) concepts. 


\subsubsection{Copeland rule}

According to the Copeland social choice rule (Copeland, 1951), the winner(s) is (are) the candidate(s), which has (have) the highest Copeland score. The Copeland score of a candidate is the number of alternatives that are beaten by that candidate.

A natural extension of this social choice rule to the social welfare framework is by ordering the alternatives based on their Copeland scores. Formally, let Cscore $(a, p)=\left|\left\{b \in A \mid a b \in T_{p}\right\}\right|$. The Copeland rule is defined as follows,

$$
\varphi^{\text {Copeland }}(p)=\{R \in \mathcal{L} \mid C(p) \subseteq R\}
$$

where $C(p)=\{a b \in A \times A \mid \operatorname{Cscore}(a, p)>C$ score $(b, p)\}$ is the ordering of the alternatives with respect to their scores. The Copeland rule chooses all linear extensions of this (possibly) weak ordering.

Proposition 3.6. Copeland rule does not satisfy WPCW, hence it does not satisfy PCW.

Proof. See Appendix B.2.1.

Proposition 3.7. Copeland rule satisfies $W C C W$, but it does not satisfy $C C W$.

Proof. See Appendix B.2.2.

\subsubsection{Minimax rule}

According to the Minimax ${ }^{8}$ social choice rule, the winner(s) is (are) the candidate(s), which has (have) the lowest Minimax score. The Minimax score of a candidate is the maximum number of individuals who prefer another particular alternative to that candidate.

A natural extension of this social choice rule to the social welfare framework is by ordering the alternatives based on their Minimax scores. Formally, let Mscore $(a, p)=\max _{b \in A \backslash\{a\}}|\{i \in N \mid b a \in p(i)\}|$. The Minimax rule is defined as follows,

$$
\varphi^{\operatorname{Minimax}}(p)=\{R \in \mathcal{L} \mid M(p) \subseteq R\},
$$

\footnotetext{
${ }^{8}$ Black, Newing, McLean, McMillan, and Monroe (1958); Simpson (1969); Kramer (1977)
} 
where $M(p)=\{a b \in A \times A \mid M \operatorname{score}(a, p)<M$ score $(b, p)\}$ is the ordering of alternatives with respect to their scores. The Minimax rule chooses all linear extensions of this (possibly) weak ordering.

Proposition 3.8. The Minimax Rule does not satisfy WPCW, hence it does not satisfy $P C W$.

Proof. See Appendix B.3.1.

Proposition 3.9. The Minimax Rule does not satisfy WCCW.

Proof. See Appendix B.3.2.

\subsubsection{Scoring rules}

A scoring vector is a vector $s=\left(s_{1}, s_{2}, \cdots, s_{m}\right) \in \mathcal{R}^{m}$ with $s_{1}>s_{2}>\cdots>s_{m}$.

For each agent within a profile, $s_{1}$ is assigned to the alternative which is ranked first, $s_{2}$ will be assigned to the alternative that is ranked second, and so on. Then, those scores are summed up. Formally, Score $(a, p)=$ $\sum_{i \in N} s_{\text {rank }(a, p(i))}$. Given a scoring vector $s$, a scoring rule is defined as follows,

$$
\varphi^{S C}(p)=\{R \in \mathcal{L} \mid S(p) \subseteq R\}
$$

where $S(p)=\{a b \in A \times A \mid \operatorname{Score}(a, p)>\operatorname{Score}(b, p)\}$ is the ordering of alternatives with respect to their scores. The specific scoring rule chooses all linear extensions of this (possibly weak) ordering. Note that Borda social welfare rule ${ }^{9}$ corresponds to a special case of scoring rules in which $s_{k}-s_{k-1}$ is constant for any $k \in\{2, \cdots, m\}$.

If the scoring vector is not strictly decreasing, we call such it degenerate, and the corresponding rule will be called as a degenerate scoring rule. Note that, a degenerate scoring rule with the scoring vector $s=(1,0, \cdots, 0)$ will order alternatives in same way as the plurality rule, which is one of the most well known social choice rules. Similarly, using scoring vector $s=(1,1, \cdots, 1,0)$ will be analogous to the anti-plurality rule.

Proposition 3.10. Scoring rules satisfy PCW, hence they satisfy WPCW.

\footnotetext{
${ }^{9}$ de Borda (1781); Black, Newing, McLean, McMillan, and Monroe (1958)
} 
Proof. See Appendix B.4.1.

Proposition 3.11. Degenerate scoring rules satisfy WPCW, but they do not satisfy $P C W$.

Proof. See Appendix B.4.2.

Proposition 3.12. (Degenerate) Scoring rules do not satisfy $W C C W$, hence they don't satisfy $C C W$.

Proof. See Appendix B.4.3.

\subsubsection{Kemeny rule}

The notion of the Kemeny distance between two linear orders can be extended to the distance between a linear order and a profile. That is the distance of a linear order to a profile is the sum of the Kemeny distances of the linear order to each individual's preference. The outcome of the Kemeny rule is the set of linear orders which minimizes this distance to the profile. Formally, the Kemeny rule is defined as follows,

$$
\varphi^{\text {Kemeny }}(p)=\left\{R \in \mathcal{L} \mid \delta(p, R) \leq \delta\left(p, R^{\prime}\right) \text { for all } R^{\prime} \in \mathcal{L}\right\}
$$

in which $\delta(p, R)=\sum_{i \in N} \delta(p(i), R)$.

Proposition 3.13. The Kemeny Rule satisfies WPCW, but it does not satisfy $P C W$.

Proof. See Appendix B.5.1.

Proposition 3.14. The Kemeny Rule satisfies $C C W$ and $W C C W$.

Proof. See Appendix B.5.2

\subsubsection{Slater rule}

Note that, the definition of the Kemeny distance for two linear orders naturally extends to any two binary relations. For any $R, R^{\prime} \subset A \times A$, the Kemeny distance is defined as: $\delta\left(R, R^{\prime}\right)=\left(\left|R \backslash R^{\prime}\right|+\left|R^{\prime} \backslash R\right|\right) / 2$.

For any given profile, the Slater rule ${ }^{10}$ finds the closest linear order(s) to

\footnotetext{
${ }^{10}$ See Slater (1961); Nurmi (2014)
} 
its corresponding pairwise tournament.

Formally, the Slater rule is:

$$
\varphi^{S}(p)=\underset{R \in \mathcal{L}(A)}{\arg \min } \delta\left(R, T_{p}\right)
$$

Proposition 3.15. The Slater Rule does not satisfy WPCW, hence it does not satisfy $P C W$.

Proof. See Appendix B.6.1

Proposition 3.16. The Slater Rule satisfies $C C W$ and $W C C W$.

Proof. See Appendix B.6.2

Table 3.1 shows a summary of the results in this section.

Table 3.1: Summary of the results

\begin{tabular}{c|c|c|c|c} 
& PCW & WPCW & CCW & WCCW \\
\hline \hline Scoring Rules & $\checkmark$ & $\checkmark$ & $\times$ & $\times$ \\
\hline Degenerate Scoring Rules & $\times$ & $\checkmark$ & $\times$ & $\times$ \\
\hline Kemeny Rule & $\times$ & $\checkmark$ & $\checkmark$ & $\checkmark$ \\
\hline Slater Rule & $\times$ & $\times$ & $\checkmark$ & $\checkmark$ \\
\hline Copeland Rule & $\times$ & $\times$ & $\times$ & $\checkmark$ \\
\hline Minimax Rule & $\times$ & $\times$ & $\times$ & $\times$
\end{tabular}

\subsection{Conclusion}

We have shown that the incompatibility between Condorcet criterion and participation criterion extends to the framework of social welfare rules. It seems that the only way to escape this incompatibility is weakening the participation criterion. For this weaker participation requirement, we consider agents who might be okay with losing in some of the pairs they won before their arrival, as long as the additional gains they have are more than what is lost. 
A straightforward research direction would be to check whether our only possibility result is actually a characterization. We suspect that together with some other axioms, weaker participation criteria and one of the Condorcet criteria may characterize the Kemeny rule. Another direction is to construct parallel criteria in social welfare framework to other participation criteria that are introduced in Jimeno, Pérez, and García (2009) and to see whether related results obtained in that paper can also be extended to the framework of social welfare rules. 


\section{Chapter 4}

\section{How to compare rulers}

\subsection{Introduction}

Consider a family deciding which fruit to eat. Possible alternatives are: $(A)$ pples, (B)ananas and (C)herries. Each family member has some strict ranking about these fruits, for example the father of the family likes bananas best, then apples, lastly cherries. We denote this by $B \succ A \succ C$. Similarly, let's say mother has $A \succ B \succ C$ and the kid has $A \succ C \succ B$. Social choice theory is concerned with aggregating such preferences over different issues.

If we approach this problem with a social choice function, one of the alternatives will be selected. Focusing on a specific representation of anyone's preference relation, we know what would be the resulting utility an agent will get given any social choice function. Using specific utilities simplifies two other applications: Evaluating strategy-proofness of functions and testing the validity of a theory with economic experiments.

Given a specific social choice function, any agent can easily check what he would be getting with a strategic vote. Then to check whether a social choice function is strategy-proof (i.e., it does not allow any profitable misreports of one's own preferences) or not will be reduced to comparing resulting alternatives by a truthful vote with any possible manipulation by any of the agents with their preference. Comparison of results using the preferences of the agents can 
be seen in the definition of strategy-proofness in Gibbard (1973); Satterthwaite (1975) and in the concept of no-show paradox as defined in Fishburn and Brams (1983).

In a similar way, within the experimental economics literature, using induced preferences as in Smith (1976) attaches some numerical values to specific alternatives. Using those values, translating any election result into monetary awards to test hypotheses related to social choice functions is straightforward. For example, Eckel and Holt (1989) test strategic voting in successive election rounds, and found that experience in a voting environment has more effect on strategic voting than knowledge about other voters. Grosser and Schram (2006) analyzes the effect of social embeddedness in voter turnout. Levine and Palfrey (2007) test three hypotheses also about voter turnout and confirms all of them. Tyszler and Schram (2016) try to explain actions of voters when there is a Condorcet cycle using Quantal Response Equilibrium.

Using social welfare functions, an order over alternatives will be selected instead of a specific alternative. It is a concept which is harder to interpret, and this difficulty shows itself in the two exemplary applications mentioned above. For example, within the experimental economics literature, any induced preference will be about the alternatives themselves, leaving the question of how to incentivize ranks as outcomes unanswered. For this, using the payment for the topmost alternative may be an easy way out, but this reduces the social welfare function to a social choice function again, evading the question.

When we turn our attention to strategy-proofness of social welfare functions, again this is harder than just comparing resulting alternatives. When formalizing the comparison done by the agents, the "metrizability" property, which implies agents behave as if to minimize some metric when comparing different rankings, is commonly assumed. Based on that, various metrics or semi-metrics can be used. Since any ranking essentially can be seen as a permutation of the original ranking of the agent, any metric over permutations will be useful. Some exemplary metrics belonging to this class are: Hamming distance, Spearman footrule distance, Kemeny (Kendall) distance among others. ${ }^{1}$ Laffond, Lainé,

\footnotetext{
${ }^{1}$ Check Golenko-Ginzburg (1991); Deza and Deza (2009) for definitions of mentioned distances for permutations, and other distances between permutations. See Blin (1976); Cook
} 
and Sanver (2018) provides a characterization for all metrizable preferences over preferences.

Among all possible distances, Kemeny distance (Kemeny, 1959) deserves extra attention. It is characterized by intuitive axioms ${ }^{2}$, hence it is widely used social choice theory. For example, Sato (2013a) considers the case where votes of agents are limited by honesty concerns, and these concerns are implemented using Kemeny distance. While defining strategy-proofness, Bossert and Storcken (1992); Athanasoglou (2016); Can, Csóka, and Ergin (2017) assume that agents compare different rankings using Kemeny distance, that is they prefer a ranking over another if their distance to the former is less.

Assuming that agents will prefer being represented by rankings "closer" to their own ranking, intuitively makes sense. However, this may not be sufficient justification for using distances that naturally takes any ranking just as a string of letters. ${ }^{3}$ Although $A B C$ is of course a string, it expresses more than that in the framework of social choice theory. Comparison between different rankings purely by distances might be at odds with a consequentialist viewpoint. While there might be an intrinsic value of being as accurately as possible represented, if there is no difference in the assigned alternative at the end, why should agents care about which specific ranking is used?

One possibly more justified way to compare rankings is introduced in Grandmont (1978), and used in Bossert and Sprumont (2014) when dealing with manipulability of aggregation rules. The framework they use implies that an agent will prefer a ranking over some other, if the former is "between" the true preference of the agent and the latter preference. ${ }^{4}$ This argument is based on what each preference will imply given a feasible nonempty subset of the alternatives. Although this is intuitive, and already has some implications for quantifying the outcomes, the order implied by this concept is not complete, and Seiford (1982); Armstrong, Cook, and Seiford (1982); Cook and Kress (1985); Monjardet $(1997,1998)$ for various (semi-) metrics between rankings.

${ }^{2}$ Kemeny and Snell (1962) provides proof for the characterization of the distance. See Can and Storcken (2018) for a refined version.

${ }^{3}$ While "social distance" concept (Akerlof, 1997) could be seen as relevant, it does not have a characterization in the sense evaluated here.

${ }^{4}$ Doğan and Lainé (2016) consider all possible extensions of preferences, and use betweenness as a domain constraint. 
that is there are pairs of preferences for which no comparison can be made. In the rest of this chapter, this incompleteness will be dealt with. To that end, Arrow (1951) includes some leads about how to translate a social welfare function into a utility function in the usual sense.

[...] sometimes not all of the [...] alternatives will be available. In analogy with the usual utility analysis of the individual consumer under conditions of constant wants and variable price-income situations, rational behavior on the part of the community would mean that the community orders the three alternatives according to its collective preferences once for all, and then chooses in any given case that alternative among those actually available which stands highest on this list.

This argument puts things into a more probabilistic setting, in which the alternative which will be reached at the end will depend on different available alternatives. The most straightforward way to formalize this idea will be using a von Neumann-Morgenstern utility function (Von Neumann and Morgenstern, 1953) to represent the order over orders. According to this, in each case the alternative which is maximal with respect to the considered ranking will be reached, and voters will prefer a ranking with a higher expected utility. This partially brings our discussion to the area of probabilistic social choice theory (Hildreth, 1953; Zeckhauser, 1969; Fishburn, 1972; Intriligator, 1973). While the use of expected utility theory within social choice theory is not new, the focus on formalizing comparison between orders, from a purely consequentialist perspective is.

Returning to our previous example, let us suppose the aggregated ranking

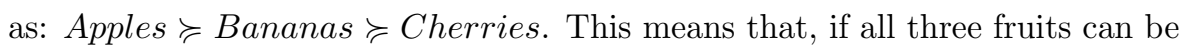
found in the market, the family will eat Apples. Since they already reached a conclusion about the order, in some other day in which Apples are not available, they will get Bananas instead. Given a probability distribution about availability (and coavailability) of each option, using utility functions of each family member, individual expected utilities related to all available orders could be derived. This will imply the comparison between different 
"rulers", and can be naturally used to monetize preferences over rankings in experiments.

In Section 4.2 our general framework and a way to compare rankings will be introduced. In Section 4.3 we characterize the cases where the introduced method is equivalent to a semi-metric. In Section 4.4 we compare the introduced method and using the Kemeny distance. We conclude in Section 4.5 with some clarifications of the framework, and future avenues for research.

\subsection{Model}

\subsubsection{General Framework}

Let $A=\left\{a_{1}, \cdots, a_{m}\right\}$ be a non-empty and finite set of alternatives. Let $\mathcal{L}$ denote the set of all possible linear orders (strict rankings) over $A$, i.e., complete, transitive, reflexive and antisymmetric binary relations over $A$. For a generic $R \in \mathcal{L}$, and any two alternatives $a_{i}, a_{j} \in A,\left(a_{i}, a_{j}\right) \in R$ is interpreted as " $a_{i}$ is at least as good as $a_{j}$ at $R$ ". For $i \neq j$, the relation is strict. To simplify notation, we write $a_{i} a_{j} \in R$ instead of $\left(a_{i}, a_{j}\right) \in R$. Similarly, for $|A|=3, R=a_{1} a_{2} a_{3}$ denotes a full ranking where $R=\left\{a_{1} a_{1}, a_{1} a_{2}, a_{1} a_{3}, a_{2} a_{2}, a_{2} a_{3}, a_{3} a_{3}\right\} . \neg R$ denotes the reverse of $R$, formally: $\neg R=\{a b \in A \times A \mid b a \in R\}$.

$R(k)$ denotes the $k^{\text {th }}$ alternative in any ranking, more formally: $R(k)=$ $a_{i} \in A$ such that $\left|\left\{a_{j} \mid a_{j} a_{i} \in R\right\}\right|=k$. For any nonempty $S \subseteq A, M(R, S)$ denotes the $R$-maximal element of $S$ with respect to $R$, that is, $a_{i} \in S$ such that for all $a_{j} \in S, a_{i} a_{j} \in R$. For any $j \in\{1, \cdots, m-1\}$ and $R, \bar{R} \in \mathcal{L}(A)$ such that $R(j)=\bar{R}(j+1), R(j+1)=\bar{R}(j)$ and $R(i)=\bar{R}(i)$ for all $i \in$ $\{1,2, \cdots, m\} \backslash\{j, j+1\}$, we say $R$ is an elementary change of $\bar{R}$ in position $j$. Using the convention in Bossert and Sprumont (2014), for preferences $R, R^{\prime}$ and $R^{\prime \prime}$, if $R \cap R^{\prime} \subseteq R^{\prime \prime} \subseteq R \cup R^{\prime}$, then we say $R^{\prime \prime}$ is in between $R$ and $R^{\prime}$, and write $R^{\prime \prime} \in\left[R, R^{\prime}\right] . \quad R^{\prime \prime} \in\left(R, R^{\prime}\right)$ carries also the meaning that $R^{\prime \prime}$ is different from both $R$ and $R^{\prime} . R^{\prime \prime} \in\left(R, R^{\prime}\right]$ and $R^{\prime \prime} \in\left[R, R^{\prime}\right)$ have the obvious interpretations. A path from $R_{1}$ to $R_{k}$ with a length of $k$ is a sequence of preferences $R_{1}, R_{2}, \cdots, R_{k}$ with each $R_{i}$ adjacent to $R_{i+1}$ in the sense that $R_{i}$ and $R_{i+1}$ are elementary changes of each other. Note that, $R^{\prime \prime}$ is between $R$ 
and $R^{\prime}$ if and only if there exist a path of minimal length from $R$ to $R^{\prime}$ through $R^{\prime \prime}$.

We are interested in lotteries over pairs of a ranking $R$ and a subset of $A$. These lotteries will be denoted as $\sigma: \mathcal{L}(A) \times 2^{A} \rightarrow[0,1]$, where $\sum_{R, S} \sigma(R, S)=1$. Here, $R$ is interpreted as the collective ordering, or ranking of a ruler that is used to make a selection from an available set. We denote all possible lotteries as mentioned by $\Omega$. An agent with preference $p \in \mathcal{L}(A)$ orders these lotteries by $\succeq_{p}$. In Axiom 4.5, the connection with $p$ will appear. But first, we define properties for any arbitrary $p$.

Axiom 4.1 (Completeness). For all $\sigma, \tau \in \Omega, \sigma \succeq_{p} \tau$ or $\tau \succ_{p} \sigma$.

Axiom 4.2 (Transitivity). For all $\sigma, \tau, v \in \Omega$, if $\sigma \succeq_{p} \tau$ and $\tau \succeq_{p} v$ then $\sigma \succeq_{p} v$.

Axiom 4.3 (Continuity). For all $\sigma, \tau, v \in \Omega$ with $\sigma \succeq_{p} \tau \succeq_{p} v$, there exists $c \in[0,1]$ such that

$$
c \sigma+(1-c) v \sim_{p} \tau
$$

Axiom 4.4 (Independence). For all $\sigma, \tau, v \in \Omega$ and for any $c \in[0,1]$ we have:

$$
c \sigma+(1-c) v \succeq_{p} c \tau+(1-c) v \Leftrightarrow \sigma \succeq_{p} \tau
$$

Remark 4.1. By Von Neumann and Morgenstern (1953), a preference relation $\succeq_{p}$ satisfies Axioms 4.1-4.4 if and only if there exists $w: \mathcal{L}(A) \times 2^{A} \rightarrow \mathbb{R}$ such that $\sigma \succeq_{p} \tau \Leftrightarrow E w(\sigma) \geq E w(\tau)$ for all $\sigma, \tau \in \Omega$ where

$$
E w(\sigma)=\sum_{(R, S) \in \mathcal{L}(A) \times 2^{A}} \sigma(R, S) w(R, S) \text { for all } \sigma \in \Omega \text {. }
$$

Next, we focus our attention on ordering of $(R, S)$ pairs, which might be understood as degenerate lotteries that assign 1 probability to these pairs. Since each pair consists of a ranking and a set, the literature which extends preferences over alternatives to preferences over sets of those alternatives is 
relevant. ${ }^{5}$ More specifically, when sets of alternatives are seen as opportunity sets, Jones and Sugden (1982), Sugden (1998), Pattanaik and Xu (1998) and Foster (2010) consider a set of reasonable preferences over alternatives to compare sets. Similarly, Kreps (1979) and Arrow (1995) approach a similar problem with agents that have uncertainty about their preferences in the time of valuation. While the mentioned literature uses different possible rankings to rank sets of alternatives, here pairs of rankings and sets are ordered.

Recall that $M(R, S)$ denotes the maximal element of $S$ according to $R$. The next axiom suggests that for a ranking $R$, an agent will compare non empty sets only by comparing $R$-maximal elements, while the one after that says that any selection is better than no selection at all.

Axiom 4.5 (Ruler Sovereignty). For any $\left(R_{1}, S_{1}\right),\left(R_{2}, S_{2}\right) \in \mathcal{L}(A) \times\left(2^{A} \backslash\right.$ $\emptyset)$,

$$
\left(R_{1}, S_{1}\right) \succeq_{p}\left(R_{2}, S_{2}\right) \Leftrightarrow\left(M\left(R_{1}, S_{1}\right), M\left(R_{2}, S_{2}\right)\right) \in p
$$

Axiom 4.6 (Willingness to Choose). For any $R_{1}, R_{2} \in \mathcal{L}(A)$, and any $S \in 2^{A}$,

$$
\left(R_{1}, S\right) \succeq_{p}\left(R_{2}, \emptyset\right)
$$

Remark 4.2. These two axioms partition $\mathcal{L}(A) \times 2^{A}$, into $m+1$ equivalence classes using $\sim_{p}$. These equivalence classes have the following properties:

- $\forall(R, S) \in \mathcal{L}(A) \times\left(2^{A} \backslash \emptyset\right), \forall p \in \mathcal{L}(A), \exists a \in A:(R, S) \sim_{p}(R,\{a\})$

- $\forall p, R_{1}, R_{2} \in \mathcal{L}(A), \forall a \in A,\left(R_{1},\{a\}\right) \sim_{p}\left(R_{2},\{a\}\right)$.

- $\forall R_{1}, R_{2} \in \mathcal{L}(A),\left(R_{1}, \emptyset\right) \sim_{p}\left(R_{2}, \emptyset\right)$.

\subsubsection{Focus In This Chapter}

In the previous section, we introduced the framework that allows any kind of lotteries over pairs of subsets and rankings. This chapter focuses on comparison

\footnotetext{
${ }^{5}$ For a general literature review on ranking sets, see Barberà, Bossert, and Pattanaik (2004). Can, Erdamar, and Sanver (2009) approaches the problem with the expected utility framework for a given ranking.
} 
between rankings, under possible subsets of $A$, interpreted as different future availabilities. To formalize this idea, we narrow down the framework by restricting our attention to a specific kind of lotteries which we denote with $\sigma_{R}$, with $R$ denoting the ranking this lottery corresponds to.

We define these lotteries with two properties.

1. For any $R, \sigma_{R}$ assigns positive probabilities only to pairs with $R$, that is:

$$
\sigma_{R}: \mathcal{L}(A) \times 2^{A} \rightarrow[0,1] \text { such that } \forall R^{\prime} \neq R, \forall S \in 2^{A}, \sigma_{R}\left(R^{\prime}, S\right)=0 .
$$

2. The probability structure is independent of any specific ranking. Formally:

$$
\forall S \in 2^{A}, \sigma_{R_{1}}\left(R_{1}, S\right)=\sigma_{R_{2}}\left(R_{2}, S\right)
$$

We further specify lotteries by considering the availability of different alternatives as independent events. Then, probability for any subset $S \subseteq A$ can be understood as a joint probability. $v\left(a_{i}\right)$ denotes the probability of $a_{i}$ being available and $v \in(0,1)^{m}$ denotes the vector for all such probabilities. Then, probability that is assigned to $(R, S)$ will be:

$$
\sigma_{R}(R, S)=\prod_{a_{i} \in S} v\left(a_{i}\right) \prod_{a_{i} \notin S}\left[1-v\left(a_{i}\right)\right] \text { for all }(R, S) \in \mathcal{L}(A) \times 2^{A}
$$

Below, we include all possible lotteries included in the limited framework we are working in, for $A=\{a, b\}$ and for some $v \in(0,1)^{2}$. Since there are only two rankings to consider, $a b$ and $b a$, the only lotteries we are interested in are $\sigma_{a b}$ and $\sigma_{b a}$. 
Table 4.1: Lotteries for $A=\{a, b\}$.

\begin{tabular}{lll}
\hline \hline Elements in $\mathcal{L}(A) \times 2^{A}$ & $\sigma_{a b}(R, S)$ & $\sigma_{b a}(R, S)$ \\
\hline$(a b, \emptyset)$ & $(1-v(a))(1-v(b))$ & 0 \\
$(a b,\{a\})$ & $v(a)(1-v(b))$ & 0 \\
$(a b,\{b\})$ & $(1-v(a)) v(b)$ & 0 \\
$(a b,\{a, b\})$ & $v(a) v(b)$ & 0 \\
$(b a, \emptyset)$ & 0 & $(1-v(a))(1-v(b))$ \\
$(b a,\{a\})$ & 0 & $v(a)(1-v(b))$ \\
$(b a,\{b\})$ & 0 & $(1-v(a)) v(b)$ \\
$(b a,\{a, b\})$ & 0 & $v(a) v(b)$ \\
\hline \hline
\end{tabular}

Next, instead of working with any $w: \mathcal{L}(A) \times 2^{A} \rightarrow \mathbb{R}$, we use some utility function that represents preference over alternatives. Consider any strictly positive $^{6} u^{p}$ that represents $p$, such that $u^{p}(a) \geq u^{p}(b)$ if and only if $a b \in p$ with equality only holding if $a=b$. For any $\left(R_{1}, S_{1}\right),\left(R_{2}, S_{2}\right) \in \mathcal{L}(A) \times\left(2^{A} \backslash \emptyset\right)$, relation between $u^{p}$ and $\succeq_{p}$ is:

$$
\begin{aligned}
& \left(R_{1}, S_{1}\right) \succeq_{p}\left(R_{2}, S_{2}\right) \Leftrightarrow \quad \text { From Axiom 4.5 } \\
& \left(M\left(R_{1}, S_{1}\right), M\left(R_{2}, S_{2}\right)\right) \in p \Leftrightarrow \quad \text { Since } u^{p} \text { represents } p \\
& u^{p}\left(M\left(R_{1}, S_{1}\right)\right) \geq u^{p}\left(M\left(R_{2}, S_{2}\right)\right)
\end{aligned}
$$

For all $R \in \mathcal{L}(A)$, we define $u^{p}(M(R, \emptyset))=0$. Then, we can extend any $u^{p}: A \rightarrow \mathbb{R}_{++}$to $u^{p}: \mathcal{L}(A) \times 2^{A} \rightarrow \mathbb{R}$ as $u^{p}(R, S)=u^{p}(M(R, S))$ to represent $\succeq_{p}$. Since $u^{p}$ is assumed to be strictly positive, Axiom 4.6 will be correctly reflected in this new representation. Using Axioms 4.1-4.4, we can also extend this utility function to preferences over lotteries over pairs of rankings and states as covered in Remark 4.1.

We will be using the same intensities for representing any preference, that is $u^{R}(R(i))=u^{R^{\prime}}\left(R^{\prime}(i)\right)$ for all $i \in\{1,2, \cdots, m\}$ and $R, R^{\prime} \in \mathcal{L}(A)$. Furthermore, for any $R \in \mathcal{L}(A)$, we will denote $u^{R}(R(i))=u_{i}$ with $u_{i}$ strictly decreasing in

\footnotetext{
${ }^{6}$ Note that assumption of strict positivity is in line with common incentives used in economic experiments, since inducing a ranking over alternatives is done by tying each alternative to some (positive) monetary payment.
} 
$i$. That way, $u$ can be understood as some utility function that is consistent across different preferences, with fixed numbers per rank, hence similar to Borda scores.

This gives all structure we need to be able to fully express the framework we are interested in. In the following example, rankings that are used to make a selection from a set are seen as sure outcomes, with a common probability structure across the subsets as mentioned before. Thus, for any lottery $\sigma_{R}$, cases with zero probabilities like $\left(R^{\prime}, S\right)$ with $R \neq R^{\prime}$ will not be mentioned.

Example 4.1. For $A=\{a, b, c\}$, we show lotteries $\sigma_{a b c}, \sigma_{b c a}$ and $\sigma_{c b a}$. The first column includes the relevant values for $\sigma_{a b c}$, second column includes the values for $\sigma_{b c a}$, and so on.

Table 4.2: Some exemplary lotteries

$$
\text { for }|A|=3 \text {. }
$$

\begin{tabular}{lllll}
\hline \hline State & Probability of State & $u^{p}(a b c, S)$ & $u^{p}(b c a, S)$ & $u^{p}(c b a, S)$ \\
\hline$\emptyset$ & $(1-v(a))(1-v(b))(1-v(c))$ & 0 & 0 & 0 \\
$\{a\}$ & $v(a)(1-v(b))(1-v(c))$ & $u^{p}(a)$ & $u^{p}(a)$ & $u^{p}(a)$ \\
$\{b\}$ & $(1-v(a)) v(b)(1-v(c))$ & $u^{p}(b)$ & $u^{p}(b)$ & $u^{p}(b)$ \\
$\{c\}$ & $(1-v(a))(1-v(b)) v(c)$ & $u^{p}(c)$ & $u^{p}(c)$ & $u^{p}(c)$ \\
$\{a, b\}$ & $v(a) v(b)(1-v(c))$ & $u^{p}(a)$ & $u^{p}(b)$ & $u^{p}(b)$ \\
$\{a, c\}$ & $v(a)(1-v(b)) v(c)$ & $u^{p}(a)$ & $u^{p}(c)$ & $u^{p}(c)$ \\
$\{b, c\}$ & $(1-v(a)) v(b) v(c)$ & $u^{p}(b)$ & $u^{p}(b)$ & $u^{p}(c)$ \\
$\{a, b, c\}$ & $v(a) v(b) v(c)$ & $u^{p}(a)$ & $u^{p}(b)$ & $u^{p}(c)$ \\
\hline \hline
\end{tabular}

In general, without explicitly imposing $v$, we define $u^{p}$ as the following:

$$
u^{p}\left(\sigma_{R}\right)=E u^{p}\left(\sigma_{R}\right)=\sum_{S \in 2^{A}} \sigma_{R}(R, S) u^{p}(R, S)
$$

Let $(R, v)$ denote the lottery $\sigma_{R}$ under $v$. Then we have: 


$$
u^{p}(R, v)=\sum_{S \in 2^{A}}\left(u^{p}(R, S) \prod_{a_{i} \in S} v\left(a_{i}\right) \prod_{a_{i} \notin S}\left[1-v\left(a_{i}\right)\right]\right)
$$

Note the similarity in the functional form between (4.2) and the multilinear extension of a cooperative game in Owen (1972). Emphasizing differences may be informative. Here, rather than having different players joining a coalition or not, we have different alternatives that may be or not considered by the ranking. The question of finding the value of each set is reduced to finding the relevant alternative within those sets, so values of sets in our sense are much more limited.

To be more specific, lotteries have the following structure: If the topmost alternative is available, whether there are other available alternatives or not is not relevant, since according to $R$, this alternative will be selected. In a similar vein, the second alternative in $R$ will only be relevant when the first one is not available. Likewise, the alternative which is ranked last will only be relevant if all other alternatives are not available. Which means, to calculate the payoff from a ranking, we can go one by one from the topmost alternative to the last one in the following way:

$$
u^{p}(R, v)=\sum_{i=1}^{m}\left(u^{p}(R(i)) v(R(i)) \prod_{j=1}^{i-1}[1-v(R(j))]\right)
$$

Whenever it is clear which $v$ is being used, we write $u^{p}(R)$ instead of $u^{p}(R, v)$.

Example 4.2. For $A=\left\{a_{1}, a_{2}, a_{3}, a_{4}\right\}, u^{p}\left(a_{i}\right)=5-i$ (with $\left.p=a_{1} a_{2} a_{3} a_{4}\right)$ and $v\left(a_{i}\right)=0.4$ for all $i \in\{1,2,3,4\}$,

$$
u^{p}\left(a_{1} a_{2} a_{3} a_{4}\right)=4 \cdot 0.4+3 \cdot 0.4 \cdot 0.6+2 \cdot 0.4 \cdot 0.6^{2}+1 \cdot 0.4 \cdot 0.6^{3}=2.6944
$$

and

$$
u^{p}\left(a_{4} a_{3} a_{2} a_{1}\right)=1 \cdot 0.4+2 \cdot 0.4 \cdot 0.6+3 \cdot 0.4 \cdot 0.6^{2}+4 \cdot 0.4 \cdot 0.6^{3}=1.6576 .
$$




\subsection{Expected Utility as a Semi-Metric}

Now that we have a method to use expected utility to compare rankings, in this section we examine the possible relationship of this method with the use of distances between rankings.

In the following, we assume that $v$ is a given common prior, and $v$ will be omitted from notation except when referring to a specific element in a probability vector. Furthermore, we iterate our assumption that $v \in(0,1)^{m}$. Note that with some if $v\left(a_{i}\right)=0$ then $a_{i}$ will not affect the payoff of the agent. Similarly, $v\left(a_{i}\right)=1$ means that alternative will be surely within the available set, and for any ranking, pairs such as $a_{i} a_{j}$ for some $a_{j}$ will be redundant. In a way, this minor limitation ensures that changes of utility corresponding to any changes in the ranking are non-zero.

To start our discussion about parallels and differences between the expected utility approach and the distance approach, we first consider elementary changes of rankings.

Proposition 4.1. For any $p \in \mathcal{L}$, and a utility function $u^{p}$, and any probability vector $v \in(0,1)^{m}$, if $R, R^{\prime} \in \mathcal{L}$ are elementary changes of each other, with $R \backslash R^{\prime} \in p$ then we have:

$$
u^{p}(R)>u^{p}\left(R^{\prime}\right)
$$

Proof. Without loss of generality, let us assume that $R$ is an elementary change of $R^{\prime}$ in position $k$. Note that, $\prod_{j=1}^{k-1}[1-v(R(j))]=\prod_{j=1}^{k-1}\left[1-v\left(R^{\prime}(j)\right)\right]$. Moreover, let us denote $R(k)=R^{\prime}(k+1)=a$ and $R(k+1)=R^{\prime}(k)=b$. Since these two rankings differ only in the placement of $a$ and $b$, when comparing $u(R)$ and $u\left(R^{\prime}\right)$ we can cancel out all terms except the $k^{\text {th }}$ and $(k+1)^{\text {th }}$ ones. 
Then, we have:

$$
\begin{aligned}
& u^{p}(R)-u^{p}\left(R^{\prime}\right) \\
& =(u(a) v(a)[1-(1-v(b))]-u(b) v(b)[1-(1-v(a))]) \prod_{j=1}^{k-1}[1-v(R(j))] \\
& =v(a) v(b)[u(a)-u(b)] \prod_{j=1}^{k-1}[1-v(R(j))]
\end{aligned}
$$

Since $a b \in p$, we have $u(a)>u(b)$. Since $v(x) \in(0,1)$ for any $x \in A$ we conclude that $u^{p}(R)>u^{p}\left(R^{\prime}\right)$.

This proposition has three main implications, all related with the concept of betweenness (Grandmont, 1978). Note that any $R \in \mathcal{L}$ will be on some minimal path between $p$ to $\neg p$. If we are dealing with two preferences that are on the same path, say $R$ and $R^{\prime}$, without loss of generality if we assume $R$ is closer to $p$ on this path, we know that there is a minimal path from $p$ to $R$, from $R$ to $R^{\prime}$ and from $R^{\prime}$ to $\neg p$. Invoking Proposition 4.1 for each elementary change through each of these ensures the following.

Corollary 4.1. For a given probability vector $v \in(0,1)^{m}, p \in \mathcal{L}$ and a utility function $u^{p}$,

$$
u^{p}(R)>u^{p}\left(R^{\prime}\right)
$$

Corollary 4.2. For a given probability vector $v \in(0,1)^{m}, p \in \mathcal{L}$ and a utility function $u^{p}$,

$$
\underset{R \in \mathcal{L}}{\arg \max } u^{p}(R)=p
$$

Corollary 4.3. For a given probability vector $v \in(0,1)^{m}, p \in \mathcal{L}$ and a utility function $u^{p}$,

$$
\underset{R \in \mathcal{L}}{\arg \min } u^{p}(R)=\neg p
$$


Note that, these three corollaries are in line with the framework in Bossert and Sprumont (2014). As long as probabilities for feasibility of each alternative are strictly between 0 and 1, using explicit calculations for expected utilities corresponding to each possible feasibility set ensures that an agent will strictly prefer a ranking over some other, if the former is between the latter and his original preference.

Next, we define a function that measures the utility difference between rankings, assuming the first ranking is a preference of an agent that is subject to decisions made by the second ranking. Because of Corollary 4.2, we know that the agents will get their maximum utility from their own ranking. Based on that, it is straightforward to assume that agents will try to minimize the decrease in this maximum utility. This gives the new framework comparability with the previous literature that assumes agents do try to minimize some distance.

Definition 4.1 (Dissimilarity Function). For a preference $p$, a utility function $u$ that represents $p$, and a probability vector $v, \delta_{v}^{u}: \mathcal{L}(A) \times \mathcal{L}(A) \rightarrow \mathbb{R}_{+}$is a function defined by:

$$
\delta_{v}^{u}(p, R)=u^{p}(p)-u^{p}(R)
$$

Let us define the properties of metrics. After that, we will check whether dissimilarity functions satisfy these properties, in general or under some conditions.

Definition 4.2 (Identity of Indiscernibles). For a given $u$ and a probability vector $v$, we say that a dissimilarity function $\delta_{v}^{u}$ satisfies identity of indiscernibles if for any $A$ and for all $R, R^{\prime} \in \mathcal{L}(A)$ it satisfies:

$$
\delta_{v}^{u}\left(R, R^{\prime}\right)=0 \text { if and only if } R=R^{\prime} .
$$

Definition 4.3 (Symmetry). For a given $u$ and a probability vector $v$, we say that a dissimilarity function $\delta_{v}^{u}$ satisfies symmetry if for any $A$ and for all $R, R^{\prime} \in \mathcal{L}(A)$ it satisfies:

$$
\delta_{v}^{u}\left(R, R^{\prime}\right)=\delta_{v}^{u}\left(R^{\prime}, R\right)
$$


Definition 4.4 (Triangle Inequality). For a given $u$ and a probability vector $v$, we say that a dissimilarity function $\delta_{v}^{u}$ satisfies triangle inequality if for any $A$ and for all $R, R^{\prime}, R^{\prime \prime} \in \mathcal{L}(A)$ it satisfies:

$$
\delta_{v}^{u}\left(R, R^{\prime \prime}\right) \leq \delta_{v}^{u}\left(R, R^{\prime}\right)+\delta_{v}^{u}\left(R^{\prime}, R^{\prime \prime}\right)
$$

Note that corollaries 4.1 and 4.2 already ensure that dissimilarity functions satisfy Identity of Indiscernibles. Whether or not Symmetry and Triangle Inequality are satisfied is less straightforward. Before going into proofs that gives us the structure in which these properties hold, let us mention what each of these properties mean in the current framework.

- Identity of indescernibles says that any agent will have the least dissimilarity to their own ranking, and only to that ranking.

- Symmetry says that given any agent and any ruler, the dissimilarity will be same if they switch their rankings.

- Triangle inequality implies that a dissimilarity between two rankings will not be greater than a dissimilarity with a third ranking plus dissimilarity of the third ranking with the second ranking. ${ }^{7}$

In the following, we show that symmetry will not be satisfied for any given dissimilarity function. The next Lemma proves that a dissimilarity function should be defined with a constant probability vector for symmetry to be satisfied.

Lemma 4.1. For $|A| \geq 3$, for any $u$, if $\delta_{v}^{u}$ is symmetric, then $v \in(0,1)^{m}$ is a constant vector.

Proof. We show that $\left.v\left(a_{1}\right)=v_{(} a_{2}\right)$. Equality of other values of $v$ can be proven analogously.

\footnotetext{
${ }^{7}$ This might be interpreted as superiority of direct democracy, given that the comparison includes first agent is being directly ruled over by the second agent, versus an intermediate ruler which rules over the first agent, but is ruled over by the second one. A party member that represents some voter and is led by a party leader might be an example to this.
} 
Let us define some four rankings as the following:

$$
\begin{aligned}
& R_{1}=a_{1} a_{3} a_{2} a_{4} \cdots a_{m} \\
& R_{2}=a_{3} a_{2} a_{1} a_{4} \cdots a_{m} \\
& R_{3}=a_{2} a_{3} a_{1} a_{4} \cdots a_{m} \\
& R_{4}=a_{3} a_{1} a_{2} a_{4} \cdots a_{m}
\end{aligned}
$$

with $R_{1}(i)=R_{2}(i)=R_{3}(i)=R_{4}(i)$ for all $i \in\{4, \cdots, m\}$.

Based on that, we have:

$$
\begin{aligned}
\delta_{v}^{u}\left(R_{1}, R_{2}\right) & =u_{1} v\left(a_{1}\right)\left[v\left(a_{2}\right)+v\left(a_{3}\right)-v\left(a_{2}\right) v\left(a_{3}\right)\right]-u_{2} v\left(a_{3}\right) v\left(a_{1}\right) \\
& +u_{3} v\left(a_{2}\right)\left[v\left(a_{1}\right) v\left(a_{3}\right)-v\left(a_{1}\right)\right] \\
\delta_{v}^{u}\left(R_{2}, R_{1}\right) & =u_{1} v\left(a_{3}\right) v\left(a_{1}\right)+u_{2} v\left(a_{2}\right)\left[v\left(a_{1}\right)-v\left(a_{1}\right) v\left(a_{3}\right)\right] \\
& +u_{3} v\left(a_{1}\right)\left[v\left(a_{2}\right) v\left(a_{3}\right)-v\left(a_{3}\right)-v\left(a_{2}\right)\right]
\end{aligned}
$$

For having symmetry, we must have: $\delta_{v}^{u}\left(R_{1}, R_{2}\right)=\delta_{v}^{u}\left(R_{2}, R_{1}\right)$ which means: $u_{1}\left[v\left(a_{2}\right)-v\left(a_{2}\right) v\left(a_{3}\right)\right]+u_{2}\left[v\left(a_{2}\right) v\left(a_{3}\right)-v\left(a_{2}\right)-v\left(a_{3}\right)\right]+u_{3}\left[v\left(a_{3}\right)\right]=0$

Similarly, we also have:

$$
\begin{aligned}
\delta_{v}^{u}\left(R_{3}, R_{4}\right) & =u_{1} v\left(a_{2}\right)\left[v\left(a_{1}\right)+v\left(a_{3}\right)-v\left(a_{1}\right) v\left(a_{3}\right)\right]-u_{2} v\left(a_{3}\right) v\left(a_{2}\right) \\
& +u_{3} v\left(a_{1}\right)\left[v\left(a_{3}\right) v\left(a_{2}\right)-v\left(a_{2}\right)\right] \\
\delta_{v}^{u}\left(R_{4}, R_{3}\right) & =u_{1} v\left(a_{3}\right) v\left(a_{2}\right)+u_{2} v\left(a_{1}\right)\left[v\left(a_{2}\right)-v\left(a_{2}\right) v\left(a_{3}\right)\right] \\
& +u_{3} v\left(a_{2}\right)\left[v\left(a_{1}\right) v\left(a_{3}\right)-v\left(a_{1}\right)-v\left(a_{3}\right)\right]
\end{aligned}
$$

Again, for having symmetry, we must have: $\delta_{v}^{u}\left(R_{3}, R_{4}\right)=\delta_{v}^{u}\left(R_{4}, R_{3}\right)$ which means:

$$
u_{1}\left[v\left(a_{1}\right)-v\left(a_{1}\right) v\left(a_{3}\right)\right]+u_{2}\left[v\left(a_{1}\right) v\left(a_{3}\right)-v\left(a_{1}\right)-v\left(a_{3}\right)\right]+u_{3}\left[v\left(a_{3}\right)\right]=0
$$

Combining (4.4) and (4.5) we get:

$$
\begin{aligned}
u_{1}\left[v\left(a_{1}\right)-v\left(a_{1}\right) v\left(a_{3}\right)\right]+ & u_{2}\left[v\left(a_{1}\right) v\left(a_{3}\right)-v\left(a_{1}\right)\right] \\
& =u_{1}\left[v\left(a_{2}\right)-v\left(a_{2}\right) v\left(a_{3}\right)\right]+u_{2}\left[v\left(a_{2}\right) v\left(a_{3}\right)-v\left(a_{2}\right)\right]
\end{aligned}
$$




$$
\begin{aligned}
v\left(a_{1}\right)\left(u_{1}\left[1-v\left(a_{3}\right)\right]+u_{2}\left[v\left(a_{3}\right)-1\right]\right) & =v\left(a_{2}\right)\left(u_{1}\left[1-v\left(a_{3}\right)\right]+u_{2}\left[v\left(a_{3}\right)-1\right]\right) \\
v\left(a_{1}\right)\left(\left(u_{1}-u_{2}\right)\left[1-v\left(a_{3}\right)\right]\right) & =v\left(a_{2}\right)\left(\left(u_{1}-u_{2}\right)\left[1-v\left(a_{3}\right)\right]\right)
\end{aligned}
$$

We know that $v\left(a_{3}\right)<1, v\left(a_{1}\right) \neq 0, v\left(a_{2}\right) \neq 0$ and $u_{1}>u_{2}$, so we have: $v\left(a_{1}\right)=v\left(a_{2}\right)$.

For having symmetric dissimilarity functions, we saw that $v$ must be a constant vector with $v_{i}=\bar{v}$ for all $i \in\{1,2, \cdots, m\}$. We can incorporate this information to normalize the utility function $u$ given before in (4.3) to focus our attention to cases that we have symmetry:

$$
u^{p}(R)=\sum_{i=1}^{m} u^{p}(R(i))(1-\bar{v})^{i-1}
$$

The next Lemma implies that increase in utility levels between consecutive alternatives in preferences has to form a geometric sequence with common ratio of $1-\bar{v}$ for a constant probability of $\bar{v}$ to have a symmetric dissimilarity function.

Lemma 4.2. For $|A| \geq 3$, for any $u$, if $\delta_{v}^{u}$ is symmetric with constant probability $\bar{v}$, then for any $i \geq 3$, the following is satisfied:

$$
u_{i-2}-u_{i-1}=\frac{u_{i-1}-u_{i}}{1-\bar{v}}
$$

Proof. Take some $A$ with $|A| \geq 3$. Take any $i \geq 3$. Pick some $R \in \mathcal{L}(A)$ and denote it as: $R=a_{1} a_{2} \cdots a_{i-2} a_{i-1} a_{i} \cdots a_{m}$. Let us introduce another preference, $R^{\prime}$ with $R^{\prime}(j)=R(j)$ for all $j \in\{1,2, \cdots, m\} \backslash\{i, i-1, i-2\}$, $R^{\prime}(i)=R(i-1), R^{\prime}(i-1)=R(i-2)$ and $R^{\prime}(i-2)=R(i)$. Let us denote it 
by $R^{\prime}=a_{1} a_{2} \cdots a_{i} a_{i-2} a_{i-1} \cdots a_{m}$.

$$
\begin{aligned}
u^{R}(R)= & u_{1}+u_{2}(1-\bar{v})+u_{3}(1-\bar{v})^{2}+\cdots+u_{i-2}(1-\bar{v})^{i-3}+u_{i-1}(1-\bar{v})^{i-2}+ \\
& u_{i}(1-\bar{v})^{i-1}+u_{i+1}(1-\bar{v})^{i}+\cdots+u_{m}(1-\bar{v})^{m-1} \\
u^{R}\left(R^{\prime}\right)= & u_{1}+u_{2}(1-\bar{v})+u_{3}(1-\bar{v})^{2}+\cdots+u_{i}(1-\bar{v})^{i-3}+u_{i-2}(1-\bar{v})^{i-2}+ \\
& u_{i-1}(1-\bar{v})^{i-1}+u_{i+1}(1-\bar{v})^{i}+\cdots+u_{m}(1-\bar{v})^{m-1} \\
u^{R^{\prime}}\left(R^{\prime}\right)= & u_{1}+u_{2}(1-\bar{v})+u_{3}(1-\bar{v})^{2}+\cdots+u_{i-2}(1-\bar{v})^{i-3}+u_{i-1}(1-\bar{v})^{i-2}+ \\
& u_{i}(1-\bar{v})^{i-1}+u_{i+1}(1-\bar{v})^{i}+\cdots+u_{m}(1-\bar{v})^{m-1} \\
u^{R^{\prime}}(R)= & u_{1}+u_{2}(1-\bar{v})+u_{3}(1-\bar{v})^{2}+\cdots+u_{i-1}(1-\bar{v})^{i-3}+u_{i}(1-\bar{v})^{i-2}+ \\
& u_{i-2}(1-\bar{v})^{i-1}+u_{i+1}(1-\bar{v})^{i}+\cdots+u_{m}(1-\bar{v})^{m-1}
\end{aligned}
$$

Following from that we have:

$$
\begin{aligned}
\delta\left(R, R^{\prime}\right)= & {\left[u_{i-2}(1-\bar{v})^{i-3}+u_{i-1}(1-\bar{v})^{i-2}+u_{i}(1-\bar{v})^{i-1}\right]-} \\
& {\left[u_{i}(1-\bar{v})^{i-3}+u_{i-2}(1-\bar{v})^{i-2}+u_{i-1}(1-\bar{v})^{i-1}\right] } \\
= & (1-\bar{v})^{i-3}\left[u_{i-2} \bar{v}+u_{i-1}\left(\bar{v}-\bar{v}^{2}\right)+u_{i}\left(\bar{v}^{2}-2 \bar{v}\right)\right]
\end{aligned}
$$

And also:

$$
\begin{aligned}
\delta\left(R^{\prime}, R\right)= & {\left[u_{i-2}(1-\bar{v})^{i-3}+u_{i-1}(1-\bar{v})^{i-2}+u_{i}(1-\bar{v})^{i-1}\right]-} \\
& {\left[u_{i-1}(1-\bar{v})^{i-3}+u_{i}(1-\bar{v})^{i-2}+u_{i-2}(1-\bar{v})^{i-1}\right] } \\
= & (1-\bar{v})^{i-3}\left[u_{i-2}\left(2 \bar{v}-\bar{v}^{2}\right)-u_{i-1} \bar{v}+u_{i}\left(\bar{v}^{2}-\bar{v}\right)\right]
\end{aligned}
$$

For symmetry, we need to have the following:

$$
\begin{aligned}
\delta\left(R, R^{\prime}\right) & =\delta\left(R^{\prime}, R\right) \\
u_{i-2} \bar{v}+u_{i-1}\left(\bar{v}-\bar{v}^{2}\right)+u_{i}\left(\bar{v}^{2}-2 \bar{v}\right) & =u_{i-2}\left(2 \bar{v}-\bar{v}^{2}\right)-u_{i-1} \bar{v}+u_{i}\left(\bar{v}^{2}-\bar{v}\right) \\
u_{i-2}\left(\bar{v}^{2}-\bar{v}\right)+u_{i-1}\left(2 \bar{v}-\bar{v}^{2}\right)-u_{i} \bar{v} & =0 \\
u_{i-2}(\bar{v}-1)+u_{i-1}(2-\bar{v})-u_{i} & =0 \\
(1-\bar{v})\left(u_{i-2}-u_{i-1}\right) & =u_{i-1}-u_{i} \\
u_{i-2}-u_{i-1} & =\frac{u_{i-1}-u_{i}}{1-\bar{v}}
\end{aligned}
$$


Lemma 4.2 implies that given an $A$ with $|A|=m \geq 3$, a constant probability $\bar{v} \in(0,1)$, some $u_{m}$ and some $k>0$ we can build the utility function as:

$$
u_{i}=u_{m}+\sum_{l=1}^{m-i} \frac{k}{(1-\bar{v})^{(l-1)}}=u_{m}+k \frac{1-(1-\bar{v})^{m-i}}{\bar{v}(1-\bar{v})^{m-i-1}}
$$

Then the next Lemma ensures that the conditions derived in Lemma 4.1 and Lemma 4.2 are actually sufficient to have $\delta_{v}^{u}$ satisfy symmetry.

Lemma 4.3. For an alternative set $A$ with $|A| \geq 3$, a constant probability $\bar{v}$, and for a utility function $u$ that satisfies (4.7) for all $i \in\{1,2, \cdots, m\}, \delta_{v}^{u}$ is symmetric.

Proof. Let us take any $A$ with $|A|=m \geq 3$. Let us take a $R \in \mathcal{L}(R)$, and denote it by $R=a_{1} a_{2} \cdots a_{m}$. For some $R^{\prime} \in \mathcal{L}(R)$, let us define the bijection $\pi:\{1,2, \cdots, m\} \leftrightarrow\{1,2, \cdots, m\}$ such that $R^{\prime}(i)=R(\pi(i))$. Based on what is being given, and dividing each utility by $\bar{v}$ as mentioned before:

$$
\begin{aligned}
u^{R}(R) & =\sum_{i=1}^{m} u_{i}(1-\bar{v})^{i-1} \\
u^{R}\left(R^{\prime}\right) & =\sum_{i=1}^{m} u_{\pi(i)}(1-\bar{v})^{i-1} \\
u^{R^{\prime}}\left(R^{\prime}\right) & =\sum_{i=1}^{m} u_{i}(1-\bar{v})^{i-1} \\
u^{R^{\prime}}(R) & =\sum_{i=1}^{m} u_{i}(1-\bar{v})^{\pi(i)-1}
\end{aligned}
$$

To show that $\delta$ is symmetric, we need to show that $\delta_{v}^{u}\left(R, R^{\prime}\right)$ and $\delta_{v}^{u}\left(R^{\prime}, R\right)$ are equal to each other. Note that, $\delta_{v}^{u}\left(R, R^{\prime}\right)=u^{R}(R)-u^{R}\left(R^{\prime}\right)$ and $\delta_{v}^{u}\left(R^{\prime}, R\right)=$ $u^{R^{\prime}}\left(R^{\prime}\right)-u^{R^{\prime}}(R)$. Since the first terms are equal to each other by (4.9) and (4.11), we only need to show $u^{R}\left(R^{\prime}\right)=u^{R^{\prime}}(R)$.

We will incorporate (4.8) in (4.10) and (4.12) in the next steps. We also assume the condition implied in (4.7) with $u(R(m))=u_{m}$ and $u_{m-1}-u_{m}=k$. 


$$
\begin{aligned}
& u^{R}\left(R^{\prime}\right)=\sum_{i=1}^{m} u_{\pi(i)}(1-\bar{v})^{i-1} \\
& =\sum_{i=1}^{m}\left(u_{m}+\frac{k}{\bar{v}} \frac{1-(1-\bar{v})^{m-\pi(i)}}{(1-\bar{v})^{m-\pi(i)-1}}\right)(1-\bar{v})^{i-1} \\
& =\sum_{i=1}^{m}\left(u_{m}(1-\bar{v})^{i-1}+\frac{k}{\bar{v}} \frac{1-(1-\bar{v})^{m-\pi(i)}}{(1-\bar{v})^{m-\pi(i)-i}}\right) \\
& =u_{m} \sum_{i=1}^{m}(1-\bar{v})^{i-1}+\frac{k}{\bar{v}} \sum_{i=1}^{m}\left(\frac{1}{(1-\bar{v})^{m-\pi(i)-i}}-(1-\bar{v})^{i}\right) \\
& =\left(u_{m}-\frac{k(1-\bar{v})}{\bar{v}}\right) \sum_{i=1}^{m}(1-\bar{v})^{i-1}+\frac{k}{\bar{v}} \sum_{i=1}^{m}\left(\frac{1}{(1-\bar{v})^{m-\pi(i)-i}}\right) \\
& u^{R^{\prime}}(R)=\sum_{i=1}^{m} u_{i}(1-\bar{v})^{\pi(i)-1} \\
& =\sum_{i=1}^{m}\left(u_{m}+\frac{k}{\bar{v}} \frac{1-(1-\bar{v})^{m-i}}{(1-\bar{v})^{m-i-1}}\right)(1-\bar{v})^{\pi(i)-1} \\
& =\sum_{i=1}^{m}\left(u_{m}(1-\bar{v})^{\pi(i)-1}+\frac{k}{\bar{v}} \frac{1-(1-\bar{v})^{m-i}}{(1-\bar{v})^{m-\pi(i)-i}}\right) \\
& =u_{m} \sum_{i=1}^{m}(1-\bar{v})^{\pi(i)-1}+\frac{k}{\bar{v}} \sum_{i=1}^{m}\left(\frac{1}{(1-\bar{v})^{m-\pi(i)-i}}-(1-\bar{v})^{\pi(i)}\right) \\
& =\left(u_{m}-\frac{k(1-\bar{v})}{\bar{v}}\right) \sum_{i=1}^{m}(1-\bar{v})^{\pi(i)-1}+\frac{k}{\bar{v}} \sum_{i=1}^{m}\left(\frac{1}{(1-\bar{v})^{m-\pi(i)-i}}\right)
\end{aligned}
$$

Note that the second terms and the coefficients of the first terms of (4.13) and (4.14) are the same. Since $\sum_{i=1}^{m}(1-\bar{v})^{\pi(i)-1}=\sum_{i=1}^{m}(1-\bar{v})^{i-1}$, the proof is complete.

Corollary 4.4. For a given set of alternatives $A, \delta_{v}^{u}$ is symmetric if and only if:

1. $v$ is a constant vector with $v=(\bar{v}, \cdots, \bar{v})$. 
2. For any $i \in\{1,2, \cdots,|A|\}$,

$$
u_{i}-u_{i+1}=\frac{u_{i+1}-u_{i+2}}{1-\bar{v}} .
$$

Example 4.3. We will be reusing the numbers from Example 4.2 to give an example for what happens when only the first condition in Corollary 4.4 is satisfied. Note that, definition of the second condition is dependent on having a constant probability.

For $A=\left\{a_{1}, a_{2}, a_{3}, a_{4}\right\}, u^{R}(R(i))=5-i$ and $v\left(a_{i}\right)=0.5$ for all $i \in$ $\{1,2,3,4\}, R_{1}=a_{1} a_{2} a_{3} a_{4}$ and $R_{2}=a_{1} a_{3} a_{4} a_{2}$ we have:

$$
\begin{aligned}
& u^{R_{1}}\left(R_{1}\right)=3.0625 \\
& u^{R_{1}}\left(R_{2}\right)=2.8125 \\
& u^{R_{2}}\left(R_{2}\right)=3.0625 \\
& u^{R_{2}}\left(R_{1}\right)=2.75
\end{aligned}
$$

Which implies $\delta_{v}^{u}\left(R_{1}, R_{2}\right) \neq \delta_{v}^{u}\left(R_{2}, R_{1}\right)$.

Next, we show that there does not exist any dissimilarity function that satisfies the triangle inequality.

Proposition 4.2. For an alternative set $A$ with $|A| \geq 3$, there exists no $(u, v)$ that makes $\delta_{v}^{u}$ satisfy the triangle inequality.

Proof. Take some $A$ with $|A|=m \geq 3$. Take any $i \geq 3$. Pick some $R, R^{\prime}, R^{\prime \prime} \in \mathcal{L}(A)$ and denote them as:

$$
\begin{aligned}
R & =a_{1} a_{2} a_{3} \cdots a_{m} \\
R^{\prime} & =a_{2} a_{3} a_{1} \cdots a_{m} \\
R^{\prime \prime} & =a_{2} a_{1} a_{3} \cdots a_{m}
\end{aligned}
$$

with $R^{\prime}(i)=R^{\prime \prime}(i)=R(i)$ for all $i \in\{4, \cdots, m\}$.

We will check whether $\delta_{v}^{u}\left(R, R^{\prime}\right) \leq \delta_{v}^{u}\left(R, R^{\prime \prime}\right)+\delta_{v}^{u}\left(R^{\prime \prime}, R^{\prime}\right)$ is satisfied. For 
that, we need the values below:

$$
\begin{aligned}
\delta_{v}^{u}\left(R, R^{\prime}\right)=u^{R}(R)-u^{R}\left(R^{\prime}\right) & =u_{1} v\left(a_{1}\right)\left[v\left(a_{2}\right)+v\left(a_{3}\right)-v\left(a_{2}\right) v\left(a_{3}\right)\right] \\
& -u_{2} v\left(a_{1}\right) v\left(a_{2}\right)+u_{3} v\left(a_{1}\right) v\left(a_{3}\right)\left[v\left(a_{2}\right)-1\right] \\
\delta_{v}^{u}\left(R, R^{\prime \prime}\right)=u^{R}(R)-u^{R}\left(R^{\prime \prime}\right) & =u_{1} v\left(a_{1}\right) v\left(a_{2}\right)-u_{2} v\left(a_{1}\right) v\left(a_{2}\right) \\
\delta_{v}^{u}\left(R^{\prime \prime}, R^{\prime}\right)=u^{R^{\prime \prime}}\left(R^{\prime \prime}\right)-u^{R^{\prime \prime}}\left(R^{\prime}\right) & =u_{2} v\left(a_{1}\right) v\left(a_{3}\right)\left[1-v\left(a_{2}\right)\right]+u_{3} v\left(a_{1}\right) v\left(a_{3}\right)\left[v\left(a_{2}\right)-1\right]
\end{aligned}
$$

We can use above values the above to check whether $\delta_{v}^{u}\left(R, R^{\prime}\right) \leq \delta_{v}^{u}\left(R, R^{\prime \prime}\right)+$ $\delta_{v}^{u}\left(R^{\prime \prime}, R^{\prime}\right)$ can hold.

$$
\begin{gathered}
\delta_{v}^{u}\left(R, R^{\prime}\right) \leq \delta_{v}^{u}\left(R, R^{\prime \prime}\right)+\delta_{v}^{u}\left(R^{\prime \prime}, R^{\prime}\right) \Leftrightarrow \\
u_{1} v\left(a_{1}\right)\left[v\left(a_{2}\right)+v\left(a_{3}\right)-v\left(a_{2}\right) v\left(a_{3}\right)\right]-u_{2} v\left(a_{1}\right) v\left(a_{2}\right)+u_{3} v\left(a_{1}\right) v\left(a_{3}\right)\left[v\left(a_{2}\right)-1\right] \leq \\
u_{1} v\left(a_{1}\right) v\left(a_{2}\right)-u_{2} v\left(a_{1}\right) v\left(a_{2}\right)+u_{2} v\left(a_{1}\right) v\left(a_{3}\right)\left[1-v\left(a_{2}\right)\right]+u_{3} v\left(a_{1}\right) v\left(a_{3}\right)\left[v\left(a_{2}\right)-1\right] \\
u_{1}\left[v\left(a_{2}\right)+v\left(a_{3}\right)-v\left(a_{2}\right) v\left(a_{3}\right)\right] \leq u_{1} v\left(a_{2}\right)+u_{2} v\left(a_{3}\right)\left[1-v\left(a_{2}\right)\right] \\
u_{1} v\left(a_{3}\right)\left[1-v\left(a_{2}\right)\right] \leq u_{2} v\left(a_{3}\right)\left[1-v\left(a_{2}\right)\right]
\end{gathered}
$$

Since by construction, $v\left(a_{3}\right)\left[1-v\left(a_{2}\right)\right]$ is positive we are left with $u_{1} \leq u_{2}$ which contradicts with the structure of our utility vector.

\subsection{Comparison with Kemeny Distance}

Definition 4.5 (Kemeny Distance). For two preferences $R_{1}, R_{2} \in \mathcal{L}(A)$, the Kemeny distance is $\delta\left(R_{1}, R_{2}\right)=\left(\left|R_{2} \backslash R_{1}\right|+\left|R_{1} \backslash R_{2}\right|\right) / 2$, half of the symmetric set difference.

First we prove that there exist no combination of $u$ and $v$ that ensures dissimilarity function will always be indifferent between two rankings whenever Kemeny distance is.

Proposition 4.3. For $|A| \geq 3$, there is no $(u, v)$ pair that will satisfy for all $R, R^{\prime}, R^{\prime \prime} \in \mathcal{L}(A)$ if $\delta^{K}\left(R, R^{\prime}\right)=\delta^{K}\left(R, R^{\prime \prime}\right)$, then $\delta_{v}^{u}\left(R, R^{\prime}\right)=\delta_{v}^{u}\left(R, R^{\prime \prime}\right)$.

Proof. We will define five different rankings which will be used throughout 
the proof. Let us denote them by:

$$
\begin{aligned}
& R_{1}=a_{1} a_{2} a_{3} a_{4} \cdots a_{m} \\
& R_{2}=a_{2} a_{1} a_{3} a_{4} \cdots a_{m} \\
& R_{3}=a_{1} a_{3} a_{2} a_{4} \cdots a_{m} \\
& R_{4}=a_{2} a_{3} a_{1} a_{4} \cdots a_{m} \\
& R_{5}=a_{3} a_{1} a_{2} a_{4} \cdots a_{m}
\end{aligned}
$$

with $R_{1}(i)=R_{2}(i)=R_{3}(i)=R_{4}(i)=R_{5}(i)$ for $i \in\{4,5, \cdots, m\}$.

Since, $\delta^{K}\left(R_{1}, R_{2}\right)=\delta^{K}\left(R_{1}, R_{3}\right)=1$, we need to have $\delta_{v}^{u}\left(R_{1}, R_{2}\right)=$ $\delta_{v}^{u}\left(R_{1}, R_{3}\right)$, which also means:

$$
\begin{aligned}
u^{R_{1}}\left(R_{2}\right) & =u^{R_{1}}\left(R_{3}\right) \\
u_{1} v\left(a_{1}\right) v\left(a_{2}\right)+u_{3} v\left(a_{2}\right) v\left(a_{3}\right)\left[1-v\left(a_{1}\right)\right] & =u_{2} v\left(a_{2}\right)\left[v\left(a_{1}\right)+v\left(a_{3}\right)-v\left(a_{1}\right) v\left(a_{3}\right)\right] \\
v\left(a_{1}\right)\left(u_{1}-u_{2}\right) & =\left(u_{2}-u_{3}\right) v\left(a_{3}\right)\left[1-v\left(a_{1}\right)\right]
\end{aligned}
$$

Since, $\delta^{K}\left(R_{1}, R_{4}\right)=\delta^{K}\left(R_{1}, R_{5}\right)=2$, we need to have $\delta_{v}^{u}\left(R_{1}, R_{4}\right)=$ $\delta_{v}^{u}\left(R_{1}, R_{5}\right)$, which also means:

$$
\begin{aligned}
u^{R_{1}}\left(R_{4}\right) & =u^{R_{1}}\left(R_{5}\right) \\
u_{1} v\left(a_{1}\right) v\left(a_{2}\right)\left[1-v\left(a_{3}\right)\right]+u_{3} v\left(a_{2}\right) v\left(a_{3}\right) & =u_{2} v\left(a_{2}\right)\left[v\left(a_{1}\right)+v\left(a_{3}\right)-v\left(a_{1}\right) v\left(a_{3}\right)\right] \\
v\left(a_{1}\right)\left[1-v\left(a_{3}\right)\right]\left(u_{1}-u_{2}\right) & =\left(u_{2}-u_{3}\right) v\left(a_{3}\right)
\end{aligned}
$$

Multiplying both sides of (4.15) by $\left[1-v\left(a_{3}\right)\right]$, we get:

$$
v(a)\left(u_{1}-u_{2}\right)\left[1-v\left(a_{3}\right)\right]=\left(u_{2}-u_{3}\right) v\left(a_{3}\right)\left[1-v\left(a_{1}\right)\right]\left[1-v\left(a_{3}\right)\right]
$$

Note that, left-hand side of (4.16) and (4.17) are equal to each other, which will imply: $\left[1-v\left(a_{1}\right)\right]\left[1-v\left(a_{3}\right)\right]=1$. This implies a contradiction, since $v\left(a_{1}\right)$, $v\left(a_{3}\right) \in(0,1)$.

Next we provide a condition for probability vectors that is necessary for Kemeny distance and dissimilarity function ordering two different rankings same way, except for indifference. 
Proposition 4.4. For $|A|=m \geq 3$, for any $R, R^{\prime}, R^{\prime \prime} \in \mathcal{L}(A)$ if $\delta^{K}\left(R, R^{\prime}\right)>$ $\delta^{K}\left(R, R^{\prime \prime}\right)$, implies $\delta_{v}^{u}\left(R, R^{\prime}\right)>\delta_{v}^{u}\left(R, R^{\prime \prime}\right)$, then for all $j, k, l \in\{1,2, \cdots, m\}$,

$$
\frac{1-v\left(a_{j}\right)}{v\left(a_{j}\right)}>\frac{v\left(a_{k}\right)-2 v\left(a_{l}\right)}{v\left(a_{k}\right) v\left(a_{l}\right)}
$$

Proof. We will define 4 different rankings which will be used throughout the proof. Let us denote them by:

$$
\begin{aligned}
R_{1} & =a_{j} a_{k} a_{l} \cdots \\
R_{2} & =a_{l} a_{k} a_{j} \cdots \\
R_{3} & =a_{l} a_{j} a_{k} \cdots \\
R_{4} & =a_{k} a_{j} a_{l} \cdots
\end{aligned}
$$

with $R_{1}(i)=R_{2}(i)=R_{3}(i)=R_{4}(i)$ for $i \in\{4,5, \cdots, m\}$.

Note that $\delta^{K}\left(R_{1}, R_{3}\right)=2, \delta^{K}\left(R_{1}, R_{4}\right)=1, \delta^{K}\left(R_{2}, R_{3}\right)=1$ and $\delta^{K}\left(R_{2}, R_{4}\right)=2$. This means to have our hypothesis satisfied we need $\delta_{v}^{u}\left(R_{1}, R_{3}\right)>\delta_{v}^{u}\left(R_{1}, R_{4}\right)$ and $\delta_{v}^{u}\left(R_{2}, R_{3}\right)<\delta_{v}^{u}\left(R_{2}, R_{4}\right)$, which will imply the following:

$$
\begin{aligned}
& \left(u_{1}-u_{2}\right) v\left(a_{j}\right)\left[v\left(a_{k}\right)-v\left(a_{l}\right)\right]<\left(u_{2}-u_{3}\right) v\left(a_{l}\right)\left[v\left(a_{k}\right)-v\left(a_{j}\right) v\left(a_{k}\right)+v\left(a_{j}\right)\right] \\
& \left(u_{2}-u_{3}\right) v\left(a_{j}\right)\left[v\left(a_{k}\right)-v\left(a_{l}\right)\right]<\left(u_{1}-u_{2}\right) v\left(a_{l}\right)\left[v\left(a_{k}\right)-v\left(a_{j}\right) v\left(a_{k}\right)+v\left(a_{j}\right)\right]
\end{aligned}
$$

If we sum these up, we have:

$$
\left(u_{1}-u_{3}\right) v\left(a_{j}\right)\left[v\left(a_{k}\right)-v\left(a_{l}\right)\right]<\left(u_{1}-u_{3}\right) v\left(a_{l}\right)\left[v\left(a_{k}\right)-v\left(a_{j}\right) v\left(a_{k}\right)+v\left(a_{j}\right)\right]
$$

Since $u_{1}-u_{3}$ is positive, we have:

$$
\begin{aligned}
v\left(a_{l}\right)\left[v\left(a_{k}\right)-v\left(a_{j}\right) v\left(a_{k}\right)+v\left(a_{j}\right)\right] & >v\left(a_{j}\right)\left[v\left(a_{k}\right)-v\left(a_{l}\right)\right] \\
v\left(a_{k}\right) v\left(a_{l}\right)+v\left(a_{j}\right) v\left(a_{l}\right)-v\left(a_{j}\right) v\left(a_{k}\right) v\left(a_{l}\right) & >v\left(a_{j}\right) v\left(a_{k}\right)-v\left(a_{j}\right) v\left(a_{l}\right) \\
v\left(a_{k}\right) v\left(a_{l}\right)\left[1-v\left(a_{j}\right)\right] & >v\left(a_{j}\right)\left[v\left(a_{k}\right)-2 v\left(a_{l}\right)\right] \\
\frac{1-v\left(a_{j}\right)}{v\left(a_{j}\right)} & >\frac{v\left(a_{k}\right)-2 v\left(a_{l}\right)}{v\left(a_{k}\right) v\left(a_{l}\right)}
\end{aligned}
$$

Lastly, we suggest another condition to have Kemeny distance and dissimilarity function compare things the same way. 
Proposition 4.5. For $|A|=m \geq 3$, for any $R, R^{\prime}, R^{\prime \prime} \in \mathcal{L}(A)$ if $\delta^{K}\left(R, R^{\prime}\right)>$ $\delta^{K}\left(R, R^{\prime \prime}\right)$, implies $\delta_{v}^{u}\left(R, R^{\prime}\right)>\delta_{v}^{u}\left(R, R^{\prime \prime}\right)$, then for all $i \in\{1,2, \cdots, m\}$,

$$
\max _{a_{j}, a_{k}, a_{l} \in A}\left(\frac{v\left(a_{j}\right)\left[v\left(a_{k}\right)-v\left(a_{l}\right)\right]}{v\left(a_{l}\right)\left[v\left(a_{k}\right)-v\left(a_{j}\right) v\left(a_{k}\right)+v\left(a_{j}\right)\right]}\right)<\frac{u_{i+1}-u_{i+2}}{u_{i}-u_{i+1}}
$$

Proof. Take any $a_{j}, a_{k}, a_{l} \in A$ and any $i \in\{1,2, \cdots, m\}$. We define 3 different rankings with $R_{1}(j)=R_{2}(j)=R_{3}(j)$ holds true for $j \in\{1,2, \cdots, m\} \backslash\{i, i+$ $1, i+2\}$. Let us denote them by:

$$
\begin{aligned}
& R_{1}=\cdots a_{j} a_{k} a_{l} \cdots \\
& R_{2}=\cdots a_{l} a_{j} a_{k} \cdots \\
& R_{3}=\cdots a_{k} a_{j} a_{l} \cdots
\end{aligned}
$$

Note that $\delta^{K}\left(R_{1}, R_{2}\right)=2$ and $\delta^{K}\left(R_{1}, R_{3}\right)=1$. This means to have our hypothesis satisfied we need $\delta_{v}^{u}\left(R_{1}, R_{2}\right)>\delta_{v}^{u}\left(R_{1}, R_{3}\right)$, which will imply the following:

$$
\left(u_{i}-u_{i+1}\right) v\left(a_{j}\right)\left[v\left(a_{k}\right)-v\left(a_{l}\right)\right]<\left(u_{i+1}-u_{i+2}\right) v\left(a_{l}\right)\left[v\left(a_{k}\right)-v\left(a_{j}\right) v\left(a_{k}\right)+v\left(a_{j}\right)\right]
$$

Since $v\left(a_{k}\right)-v\left(a_{j}\right) v\left(a_{k}\right)+v\left(a_{j}\right)=v\left(a_{k}\right)\left[1-v\left(a_{j}\right)\right]+v\left(a_{j}\right)$ is positive, we have:

$$
\frac{v\left(a_{j}\right)\left[v\left(a_{k}\right)-v\left(a_{l}\right)\right]}{v\left(a_{l}\right)\left[v\left(a_{k}\right)-v\left(a_{j}\right) v\left(a_{k}\right)+v\left(a_{j}\right)\right]}<\frac{u_{i+1}-u_{i+2}}{u_{i}-u_{i+1}}
$$

The preceding propositions are also sufficent to ensure that Kemeny distance and dissimilarity function compare the rankings in the same way when $m=3$, (although we skip the proof here) but for greater dimensions, we need more and more restrictions to have the same.

\subsection{Conclusion}

Our general framework allows any kind of lotteries over pairs of rankings and subsets. Thus, in a future work, probabilistic social welfare functions can be modeled as lotteries over $\sigma_{R}$ (which themselves are lotteries) assuming again a 
common probability structure. Another generalization of the current model will be omitting the assumption of availability as independent events and directly dealing with probabilities. This, for example allows probability of $S \backslash\{a\}$ and $S \cup\{a\}$ to be independent from each other for any $S .{ }^{8}$ Lastly, as we did in Section 4.4 with Kemeny distance, formalizing parallels between the proposed method and other (semi-) metrics may be covered in future work.

Whether evaluation of rankings in real life has metric properties or not is of course still an open question, since this evaluation depends directly on how the rankings are used. Still, this chapter might be seen as a step to make assumptions about comparisons over rankings more testable. Under the proposed framework, a social welfare function can be monetized using the induced preferences framework Smith (1976), as it has been done for social choice functions already, only this time, an alternative will be selected according to the social welfare function only from a randomly selected subset of all alternatives. Using this way, both $u$ and $v$ can be parametrized within the experiment, and metric properties could be tested given this specific interpretation of social welfare functions.

Note that without the assumption of interpersonal comparability, the framework provided here can not be used as a way to aggregate preferences. Furthermore, here we are not tackling the problem of the existence of collective rationality (as pointed out in Buchanan (1954)) since we assume that the social ordering has already been decided. ${ }^{9}$ Thus, this could be seen as a tool to analyze personal opinions about governments in an ex-ante sense.

\footnotetext{
${ }^{8}$ Our preliminary efforts lead us to suggest that, under this more generalized possibilities for subsets, Identity of Indescernibles still holds for all cases, while Symmetry is again a property that will only be obtained under special structure of probabilities and intensities. Whether we will get a possibility about Triangle Inequality is an open question, so we can not rule out a possibility of a metric accurately representing the preferences defined for more general lotteries representing rankings.

${ }^{9}$ We may evade the problem by equating social preference to a preference of some specific individual, hence the title of this chapter.
} 


\section{Bibliography}

Akerlof, G. A. (1997): "Social distance and social decisions," Econometrica: Journal of the Econometric Society, pp. 1005-1027.

Armstrong, R. D., W. D. Cook, and L. M. Seiford (1982): "Priority ranking and consensus formation: the case of ties," Management Science, 28(6), 638-645.

Arrow, K. J. (1951): Social choice and individual values. John Wiley \& Sons. (1995): "A note on freedom and flexibility," Choice, welfare and development: a festschrift in honour of Amartya K. Sen, pp. 7-15.

Athanasoglou, S. (2016): "Strategyproof and efficient preference aggregation with Kemeny-based criteria," Games and Economic Behavior, 95, 156 - 167.

BArberÀ, S. (2011): "Strategyproof Social Choice," in Handbook of Social Choice and Welfare, ed. by K. J. Arrow, A. Sen, and K. Suzumura, vol. 2 of Handbook of Social Choice and Welfare, pp. 731 - 831. Elsevier.

Barberà, S., W. Bossert, and P. K. Pattanaik (2004): "Ranking sets of objects," in Handbook of utility theory, pp. 893-977. Springer.

BarberÀ, S., B. Dutta, And A. Sen (2001): "Strategy-proof social choice correspondences," Journal of Economic Theory, 101(2), 374-394.

Black, D., R. A. Newing, I. Mclean, A. McMillan, and B. L. Monroe (1958): The theory of committees and elections. Springer. 
BLIN, J.-M. (1976): "A linear assignment formulation of the multiattribute decision problem," Revue française d'automatique, informatique, recherche opérationnelle. Recherche opérationnelle, 10(V2), 21-32.

Bossert, W., And Y. Sprumont (2014): "Strategy-proof preference aggregation: Possibilities and characterizations," Games and Economic Behavior, 85, 109-126.

Bossert, W., And T. Storcken (1992): "Strategy-proofness of social welfare functions: The use of the Kemeny distance between preference orderings," Social Choice and Welfare, pp. 345-360.

Brandt, F., C. Geist, and D. Peters (2016): "Optimal bounds for the no-show paradox via SAT solving," Mathematical Social Sciences.

Buchanan, J. M. (1954): "Social choice, democracy, and free markets," Journal of Political Economy, 62(2), 114-123.

Can, B., P. Csóka, and E. Ergin (2017): "How to choose a delegation for a peace conference?," Research Memorandum 008, Maastricht University, Graduate School of Business and Economics (GSBE).

Can, B., B. Erdamar, and M. R. Sanver (2009): "Expected utility consistent extensions of preferences," Theory and Decision, 67(2), 123-144.

Can, B., E. Ergin, and M. Pourpouneh (2017): "Condorcet versus participation criterion in social welfare rules," Research Memorandum 020, Maastricht University, Graduate School of Business and Economics (GSBE).

Can, B., And T. Storcken (2018): "A re-characterization of the Kemeny distance," Journal of Mathematical Economics.

Condorcet, MARquis DE. (1785): Essay sur l'application de l'analyse de la probabilité des decisions: redues et pluralité des voix. l'Imprimerie Royale.

- (1788): "On the constitution and the functions of provincial assemblies," Translated and excerpted in: McLean and Hewitt, 1994, 139-68. 
CoOK, W. D., And M. Kress (1985): "Ordinal ranking with intensity of preference," Management science, 31(1), 26-32.

Cook, W. D., And L. M. SEIFOrd (1982): "On the Borda-Kendall consensus method for priority ranking problems," Management Science, 28(6), 621-637.

Copeland, A. H. (1951): "A reasonable social welfare function," in Mimeographed notes from a Seminar on Applications of Mathematics to the Social Sciences, University of Michigan.

DE BordA, J. C. (1781): "Memoire sur les Elections au Scrutin," Histoire de l'Academie Royale des Sciences, Paris.

Deza, M. M., And E. Deza (2009): Encyclopedia of Distanceschap. Distances on Strings and Permutations, pp. 201-213. Springer.

DoĞAn, O., And J. LAInÉ (2016): "Strategic Manipulation of Social Welfare Functions via Strict Preference Extensions," Mimeo, 5th World Congress of the Game Theory Society.

Dudouet, V., And S. Lundström (2016): "Post-war Political Settlements: From Participatory Transition Processes to Inclusive State-building and Governance," Research report, Berghof Foundation, Berlin.

Eckel, C., and C. A. Holt (1989): "Strategic Voting in Agenda-Controlled Committee Experiments," The American Economic Review, 79(4), 763-773.

Felsenthal, D. S., And H. Nurmi (2016): "Two types of participation failure under nine voting methods in variable electorates," Public Choice, 168(1-2), 115-135.

Fishburn, P. C. (1972): "Lotteries and social choices," Journal of Economic Theory, 5(2), 189-207.

Fishburn, P. C. (1977): "Condorcet Social Choice Functions," SIAM Journal on Applied Mathematics, 33(3), 469-489.

Fishburn, P. C., And S. J. Brams (1983): "Paradoxes of preferential voting," Mathematics Magazine, 56(4), 207-214. 
Foster, J. (2010): "Notes on effective freedom," Discussion paper, Queen Elizabeth House, University of Oxford.

GibBard, A. (1973): "Manipulation of voting schemes: a general result," Econometrica: journal of the Econometric Society, pp. 587-601.

Golenko-GinzburG, D. (1991): "Metrics in the permutation space," Applied Mathematics Letters, 4(2), 5-7.

Grandmont, J.-M. (1978): "Intermediate Preferences and the Majority Rule," Econometrica, 46(2), 317-330.

Grosser, J., ANd A. Schram (2006): "Neighborhood information exchange and voter participation: An experimental study," American political science Review, 100(2), 235-248.

HildREth, C. (1953): "Alternative conditions for social orderings," Econometrica: Journal of the Econometric Society, pp. 81-94.

Intriligator, M. D. (1973): "A probabilistic model of social choice," The Review of Economic Studies, 40(4), 553-560.

Jimeno, J. L., J. Pérez, and E. García (2009): "An extension of the Moulin No Show Paradox for voting correspondences," Social Choice and Welfare, 33(3), 343-359.

Jones, P., And R. Sugden (1982): "Evaluating choice," International Review of Law and Economics, 2(1), 47-65.

Kasper, L., H. Peters, and D. Vermeulen (2017): "Condorcet Consistency and the strong no show paradoxes," Research Memorandum 017, Maastricht University, Graduate School of Business and Economics (GSBE).

Kemeny, J., And J. Snell (1962): Mathematical Models in the Social Sciences. Boston: Ginn.

Kemeny, J. G. (1959): "Mathematics without numbers," Daedalus, 88(4), $577-591$. 
Kramer, G. H. (1977): "A dynamical model of political equilibrium," Journal of Economic Theory, 16(2), 310-334.

Kreps, D. M. (1979): "A representation theorem for" preference for flexibility"," Econometrica: Journal of the Econometric Society, pp. 565-577.

Laffond, G., J. Lainé, and M. R. Sanver (2018): "Metrizable Preferences over Preferences," Mimeo.

LANZ, D. (2011): "Who gets a seat at the table? A framework for understanding the dynamics of inclusion and exclusion in peace negotiations," International Negotiation, 16(2), 275-295.

Levine, D. K., and T. R. Palfrey (2007): "The paradox of voter participation? A laboratory study," American political science Review, 101(01), 143-158.

Monjardet, B. (1997): "Concordance between two linear orders: the Spearman and Kendall coefficients revisited," Journal of classification, 14(2), 269-295.

(1998): "On the comparison of the Spearman and Kendall metrics between linear orders," Discrete Mathematics, 192(1-3), 281-292.

Moulin, H. (1988): "Condorcet's principle implies the no show paradox," Journal of Economic Theory, 45(1), 53-64.

Myint, S. Y. S., And A. Slodkowski (2016): "Myanmar's Suu Kyi kicks off peace conference with appeal for unity," https://www.reuters.com/ article/us-myanmar-politics-peace/myanmars-suu-kyi-kicks-offpeace-conference-with-appeal-for-unity-idUSKCN1160RF, Accessed: 2017-11-22.

NúÑEZ, M., and M. R. Sanver (2017): "Revisiting the connection between the no-show paradox and monotonicity," Mathematical Social Sciences, 86, $59-67$. 
NuRMi, H. (2014): "Are we done with preference rankings? If we are, then what?," Operations Research and Decisions, 24(4), 63-74.

Owen, G. (1972): "Multilinear extensions of games," Management Science, 18(5-part-2), 64-79.

Pattanaik, P. K., And Y. Xu (1998): "On preference and freedom," Theory and Decision, 44(2), 173-198.

PÉrez, J. N. (2001): "The strong no show paradoxes are a common flaw in Condorcet voting correspondences," Social Choice and Welfare, 18, 601-616.

Pitkin, H. F. (1967): The Concept of Representation. University of California Press.

SATO, S. (2013a): "Strategy-proofness and the reluctance to make large lies: the case of weak orders," Social Choice and Welfare, pp. 1-16.

- (2013b): "A sufficient condition for the equivalence of strategyproofness and nonmanipulability by preferences adjacent to the sincere one," Journal of Economic Theory, 148(1), 259-278.

Satterthwaite, M. A. (1975): “Strategy-proofness and Arrow's conditions: Existence and correspondence theorems for voting procedures and social welfare functions," Journal of economic theory, 10(2), 187-217.

Simpson, P. B. (1969): "On defining areas of voter choice: Professor Tullock on stable voting," The Quarterly Journal of Economics, 83(3), 478-490.

Slater, P. (1961): "Inconsistencies in a schedule of paired comparisons," Biometrika, 48(3/4), 303-312.

Sмith, J. H. (1973): "Aggregation of preferences with variable electorate," Econometrica, pp. 1027-1041.

Sмiтh, V. L. (1976): "Experimental economics: Induced value theory," The American Economic Review, 66(2), 274-279. 
Sugden, R. (1998): "The metric of opportunity," Economics E Philosophy, 14(2), 307-337.

Tyszler, M., AND A. Schram (2016): "Information and strategic voting," Experimental Economics, 19(2), 360-381.

Von Neumann, J., and O. Morgenstern (1953): Theory of games and economic behavior. Princeton University Press.

Young, H. P. (1974): "An axiomatization of Borda's rule," Journal of Economic Theory, 9(1), 43-52.

- (1975): "Social choice scoring functions," SIAM Journal on Applied Mathematics, 28(4), 824-838.

Young, H. P., And A. Levenglick (1978): "A consistent extension of Condorcet's election principle," SIAM Journal on applied Mathematics, $35(2), 285-300$.

Zeckhauser, R. (1969): "Majority rule with lotteries on alternatives," The Quarterly Journal of Economics, pp. 696-703. 



\section{Appendix A}

\section{Appendix for Chapter 2}

\section{A.1 Examples}

\section{A.1.1 Example for showing the Kemeny rule is not strategy-proof}

Example A.1. Given a profile $P \in \mathcal{L}(A)^{n}$, a preference $R$ is a Kemeny ranking for $P$, if for all $R^{\prime} \in \mathcal{L}(A)$, we have that $\sum_{i \in N} \delta(R, P(i)) \leq$ $\sum_{i \in N} \delta\left(R^{\prime}, P(i)\right)$. A rule which assigns all Kemeny rankings to each profile is called the Kemeny rule. More formally, the Kemeny rule, denoted by $\varphi_{K}$, assigns to a profile $P \in \mathcal{L}(A)^{n}: \varphi_{K}(P)=\{R \in L(A) \mid$ $R$ is a Kemeny ranking for $P\}$.

Our counterexample is just with 4 alternatives, and 11 agents. $P$ is as follows:

$\begin{array}{lllllllllll}d & d & d & a & c & c & a & b & b & b & b \\ a & a & a & d & a & a & b & c & c & c & c \\ b & b & c & c & b & b & d & d & d & d & a \\ c & c & b & b & d & d & c & a & a & a & d\end{array}$

It can be seen that $\varphi(P)=\{a b c d\} . \quad \delta(a b c d, b c a d)=2$. The last agent can manipulate to reach the following $P^{\prime}$ : 


$\begin{array}{lllllllllll}d & d & d & a & c & c & a & b & b & b & \mathrm{~b} \\ a & a & a & d & a & a & b & c & c & c & \mathrm{c} \\ b & b & c & c & b & b & d & d & d & d & \mathrm{~d} \\ c & c & b & b & d & d & c & a & a & a & \mathrm{a}\end{array}$

It can be seen that $\varphi\left(P^{\prime}\right)=\{b c d a\} . \quad \delta(b c d a, b c a d)=1$. So, the last agent is in a better position with reporting a false profile. So, even though both the Kemeny rule and our strategy-proofness condition is defined upon minimal Kemeny distance, we show that the Kemeny rule is not strategy-proof.

\section{A.1.2 Example for a rule which is Ballot Neutral}

Example A.2. Let us take two sets, $A$ and $B$ with $A \subsetneq \bar{A},|A|=3$ and $|\bar{A}|=4$. Let us have 7 agents. For this example, we use the lexicographic enumeration where alternatives are ordered with their place in the alphabet, so $R_{1}=a b c, R_{2}=a c b$ and so on, and $\bar{R}_{1}=a b c d, \bar{R}_{2}=a b d c$ and so on.

Let us define $P \in \mathcal{L}(A)^{7}$ as

$$
\begin{array}{lllllll}
a & a & a & a & a & b & b \\
b & b & b & c & c & a & c \\
c & c & c & b & b & c & a
\end{array} \quad \text { Here, } p=(3,2,1,1,0,0) .
$$

Take some $\varphi$ where $\varphi(P)=\{a b c\}=\left\{R_{1}\right\}$. Next, we define $\bar{P} \in \mathcal{L}(\bar{A})^{7}$ as

$\begin{array}{lllllll}a & a & a & a & a & a & a \\ b & b & b & b & b & c & c \\ c & c & c & d & d & b & d \\ d & d & d & c & c & d & b\end{array}$$$
\text { Here, } \bar{p}=(3,2,1,1, \underbrace{00 \ldots 0}_{20 \text { zeros. }}) \text {. }
$$

There is an injective function from $A$ to $\bar{A}$ that satisfies our condition. For $\pi(t)=t$, we have $p_{t}=\bar{p}_{\pi(t)}=\bar{p}_{t}$ for all $t \in\{1,2, \ldots, 6\}$. So, for $\varphi$ to satisfy ballot neutrality, we must have $\varphi(\bar{P})=\left\{\bar{R}_{\pi(1)}\right\}=\left\{\bar{R}_{1}\right\}=\{$ abcd $\}$.

Or, since ballot neutrality is binding two ways, we can claim that if $\varphi(\bar{P})=$ $\{a b c d\}=\left\{\bar{R}_{\pi(1)}\right\}=\left\{\bar{R}_{1}\right\}$, then we must have that $\varphi(P)=\{a b c\}=\left\{R_{1}\right\}$. 


\section{A.1.3 Example for a profile in which ballot neutrality is used to increase coalitional options.}

Example A.3. Consider $A=\{a, b, c\}, N=16$, and a profile $P$ whose support is $p=(5,4,3,2,1,1)$. If $\varphi(P)=\left\{R_{1}, R_{2}, R_{3}, R_{4}\right\}$, then agents whose preferences are not in the delegation have distance of 1 to the nearest delegate in all possible permutations. So, there is not a possible clustering, in which every agent strictly prefers some other non-delegate to the closest delegate for this particular alternative set. However, when we move to $\bar{A}=\{a, b, c, d\}$, it is easy to see that there is such a cluster with correct injection.

\section{A.2 Proofs}

\section{A.2.1 Proof of Proposition 2.1}

Proposition 2.1. If a rule $\varphi$ satisfies Pareto optimality and ballot neutrality, then for all $P \in \mathcal{L}(A)^{n}, \varphi(P) \subseteq R P(P)$.

Proof:. Take some finite $A \subsetneq \mathcal{A}$ and $N \subsetneq \mathcal{N}$. Take some preference profile $P \in \mathcal{L}(A)^{n}$ with $R P(P) \subseteq \mathcal{L}(A)$. First, note that with equality, we are done. Suppose, for a contradiction, that there exists a preference $R \in \varphi(P)$ with $p(R)=0$ that is, a preference with zero support. Consider any $x \in \mathcal{A} \backslash A$ and let us construct the expansion of $P$ to $\bar{A}=A \cup x$ by $\bar{P} \in \mathcal{L}(\bar{A})^{n}$ as follows: for all $i \in N, \bar{P}(i)=P(i) \| x$ where $P(i) \| x=P(i) \cup(x, x) \cup\{(a, x) \mid a \in A\}$, i.e., concatenation of $x$ with $P(i)$. Clearly, for any $a \in A$ and $i \in N,(a, x) \in \bar{P}(i)$. Take any $a \in A$, and $R^{*} \in \mathcal{L}(\bar{A})$ such that $(x, a) \in R^{*}$. By Pareto optimality, $R^{*} \notin \varphi(\bar{P})$. Note that $\bar{p}\left(R^{*}\right)=0=\bar{p}(R \| x)$. Then by ballot neutrality $R \| x \notin \varphi(\bar{P})$. As $\bar{P}$ is an expansion of $P$, again by ballot neutrality, we conclude that $R \notin \varphi(P)$.

\section{A.2.2 Proof of Proposition 2.3}

Proposition 2.3. For all $A \subsetneq \mathcal{A}, N \subsetneq \mathcal{N}, P \in \mathcal{L}(A)^{n}$, and all threshold functions $f$, the threshold rule $\varphi^{f}(P)$ is well-defined. 


\section{Proof:.}

Let us pick some threshold function $f$, and consider the corresponding threshold rule $\varphi^{f}(P)$. To show that the rule is well-defined, we need to show the following.

1. There is always a $t$ that satisfies $\rho_{t} \geq f(t)$.

For any $p \in \mathcal{L}(A)^{n}, t=|A|$ !, satisfies this. Since any reported preference should be within $\mathcal{L}(A)$, including all preferences in the delegation ensures to have cumulative support of 1 . Since by definition any $f(t) \leq 1$, we have: $\rho_{|A| !} \geq f(|A| !)$. This shows that the universal set is always guaranteed to pass the relevant threshold.

\section{There is always a unique way to pick the first $t$ preferences.}

Case 1: For all distinct $i, j$, we have $p_{i} \neq p_{j}$.

If this is the case, powers of the preferences are well ordered, there is a unique ordering enumeration, so there is always a unique way to pick the first $t$ preferences.

Case 2: For some distinct $i, j$, we have $p_{i}=p_{j}$.

We will show that for any $p_{i}=p_{j}$ it is either $R_{i}, R_{j} \in \varphi^{f}(P)$ or $R_{i}, R_{j} \notin$ $\varphi^{f}(P)$.

First, consider the case where the number of delegates is one. In that case, we must have $\rho_{1}=\frac{p_{1}}{n} \geq f(1)>\frac{1}{2}$. This directly shows that any enumeration has the same preference as its first, whenever the first preference is passing the relevant threshold.

Second, consider the case when $t^{*}>1$. By definition, for all $t>1$ we have that

$f(t) \geq \frac{1+f(t-1)}{2}$. Multiply both sides by 2 to get

$2 f(t) \geq 1+f(t-1) . \quad$ Subtract $f(t)+f(t-1)$ from both sides to get

$$
f(t)-f(t-1) \geq 1-f(t) .
$$

From definition of the rule, we know the following is true for some $t^{*}$ :

$$
\begin{aligned}
\rho_{t^{*}-1} & <f\left(t^{*}-1\right), \\
\rho_{t^{*}} & \geq f\left(t^{*}\right) .
\end{aligned}
$$


Multiplying both sides of $(\mathrm{A} .2 \mathrm{~b})$ by -1 and adding 1 to both sides we get

$$
1-\rho_{t^{*}} \leq 1-f\left(t^{*}\right)
$$

Subtracting (A.2a) from (A.2b) leads to

$$
\frac{p\left(t^{*}\right)}{n}>f\left(t^{*}\right)-f\left(t^{*}-1\right) .
$$

If we combine (A.3), (A.4), and (A.1) we get

$$
\frac{p\left(t^{*}\right)}{n}>f\left(t^{*}\right)-f\left(t^{*}-1\right) \geq 1-f\left(t^{*}\right) \geq 1-\rho_{t^{*}}=\sum_{i=t^{*}+1}^{|A| !} \frac{p_{i}}{n} .
$$

The rightmost term is the total support for the preferences which are not part of the delegation, where the leftmost term is the support for the weakest delegate. This implies that not only the weakest delegate has strictly more support than the next preference, but he also has strictly more support than the total support for non-delegates. This says that there is again a unique way to select the topmost $t$ preferences as delegates, even though the relevant enumeration is not unique this time. In other words, when there is any tie between support for some preferences, by design all of those preferences belong to the delegation or none of them.

\section{A.2.3 Proof of Lemma 2.1}

Lemma 2.1. If a rule $\varphi$ satisfies consistency, ballot neutrality, and strategyproofness, then for all $A \subsetneq \mathcal{A}, N \subsetneq \mathcal{N}$ and $P \in \mathcal{L}(A)^{n}$ if $R \in \varphi(P)$ and $p\left(R^{\prime}\right) \geq p(R)$, we have $R^{\prime} \in \varphi(P)$.

Proof:. Suppose, for a contradiction, that there exists two preferences $R^{\mathrm{h}}$ (preference with (h)igher support), and $R^{\mathrm{l}}$ (preference with (l)ower support) with $R^{\mathrm{l}} \in \varphi(P)$ and $R^{\mathrm{h}} \notin \varphi(P)$. Let $p\left(R^{\mathrm{h}}\right)=h$ and $p\left(R^{\mathrm{l}}\right)=l$. Without loss of generality, we can assume that $h+l$ is even since by consistency we can replicate the profile once by using the two-fold replica with no changes in the delegation. This will ensure that $h-l$ can also be assumed to be even. 
First, assume that $h-l=0$. This contradicts ballot neutrality since $h=l$. Next, assume that $h-l=2$. In that case, an agent whose original preference is $R^{\mathrm{h}}$ may misreport $R^{\mathrm{l}}$. Denoting the modified profile by $P^{\prime}$, that will cause $p^{\prime}\left(R^{\mathrm{h}}\right)=p^{\prime}\left(R^{\mathrm{l}}\right)$. From ballot neutrality, either both of $R^{\mathrm{h}}$ and $R^{\mathrm{l}}$ will be in the delegation, or none will be included. If both are included, this means that the agent deviated to his benefit, contradicting strategy-proofness. If none is included, some agent with $R^{\mathrm{l}}$ as his original preference may report $R^{\mathrm{h}}$ to get back to the original preference profile, resulting in $R^{\mathrm{l}} \in \varphi(P)$ again, this also contradicts strategy-proofness. So for $h-l=2$, we showed that with the original preference profile, if $R^{\mathrm{l}}$ is included in the delegation, so must $R^{\mathrm{h}}$ be.

Assume that our hypothesis holds for $h-l=k$ for some even $k$, that is, if $R^{\mathrm{l}} \in \varphi(P)$, then $R^{\mathrm{h}} \in \varphi(P)$. Now, let $h-l=k+2$. Then an agent whose original preference is $R^{\mathrm{h}}$ can report $R^{\mathrm{l}}$ to trigger the situation with $h-l=k$. Since this violates strategy-proofness, we must have $R^{\mathrm{h}} \in \varphi(P)$ even when $h-l=k+2$. By induction, this completes the proof.

\section{A.2.4 Proof of Lemma 2.2}

Lemma 2.2. If a rule $\varphi$ satisfies consistency and ballot neutrality, then for all $A \subsetneq \mathcal{A}, N, N^{\prime} \subsetneq \mathcal{N}$ and for all $P \in \mathcal{L}(A)^{n}, P^{\prime} \in \mathcal{L}(A)^{n^{\prime}}$ such that $p / n=p^{\prime} / n^{\prime}$, we have $\varphi(P)=\varphi\left(P^{\prime}\right)$.

Proof:. From consistency, we know that $\varphi(P)=\varphi(2 P)=\varphi(3 P)=\ldots=$ $\varphi(n P)$. So, $\varphi(P)=\varphi\left(n^{\prime} P\right)$ and $\varphi\left(P^{\prime}\right)=\varphi\left(n P^{\prime}\right)$. Since $n^{\prime} p=n p^{\prime}$, ballot neutrality implies that $\varphi\left(n^{\prime} P\right)=\varphi\left(n P^{\prime}\right)$, completing the proof.

\section{A.2.5 Proof of Lemma 2.3}

Lemma 2.3. If a rule $\varphi$ satisfies consistency and ballot neutrality, then for all $A \subsetneq \mathcal{A}, N \subsetneq \mathcal{N}$ and $P \in \mathcal{L}(A)^{n}$, denoting $|\varphi(P)|=t$, and picking an enumeration on $\mathcal{L}(A)$ such that $p_{i} \geq p_{j}$ for all $i<j$, the following holds:

i) For any $P^{\prime} \in \mathcal{L}(A)^{n}$ such that $\frac{p_{j}^{\prime}}{n}=\sum_{i=1}^{t} \frac{p_{i}}{n t}$ for all $j \in\{1,2, \ldots, t\}$ and $\frac{p_{j}^{\prime}}{n}=\frac{p_{j}}{n}$ for all $j \in\{t+1, t+2, \ldots,|A|$ ! $\}$ we have $\varphi(P)=\varphi\left(P^{\prime}\right)$. 
ii) For any $P^{\prime \prime} \in \mathcal{L}(A)^{n}$ such that $\frac{p_{j}^{\prime \prime}}{n}=\frac{p_{j}}{n}$ for all $j \in\{1,2, \ldots, t\}$ and $\frac{p_{j}^{\prime \prime}}{n}=$ $\sum_{i=t+1}^{|A| !} \frac{p_{j}}{n(|A| !-t)}$ for all $j \in\{t+1, t+2, \ldots,|A| !\}$ we have $\varphi(P)=\varphi\left(P^{\prime \prime}\right)$.

\section{Proof:.}

i) Let $P$ and $p=\left(p_{1}, p_{2}, \ldots, p_{|A| !}\right)$ be as in the Lemma with $|\varphi(P)|=t$ and $P^{\prime}$ as defined in the Lemma. Consider the following profiles with the same enumeration on $\mathcal{L}(A)$ where $p_{1}, p_{2}, \ldots, p_{t}$ rotates and bold numbers indicate the support for the chosen delegates:

$$
\begin{gathered}
P^{1} \in \mathcal{L}(A)^{n} \text { such that } p^{1}=\left(\mathbf{p}_{\mathbf{t}}, \mathbf{p}_{\mathbf{1}}, \mathbf{p}_{\mathbf{2}}, \ldots, \mathbf{p}_{\mathbf{t}-\mathbf{1}}, p_{t+1}, p_{t+2}, \ldots, p_{|A| !}\right) \\
P^{2} \in \mathcal{L}(A)^{n} \text { such that } p^{2}=\left(\mathbf{p}_{\mathbf{t}-\mathbf{1}}, \mathbf{p}_{\mathbf{t}}, \mathbf{p}_{\mathbf{1}}, \ldots, \mathbf{p}_{\mathbf{t}-\mathbf{2}}, p_{t+1}, p_{t+2}, \ldots, p_{|A| !}\right)
\end{gathered}
$$

$$
P^{t-1} \in \mathcal{L}(A)^{n} \text { such that } p^{t-1}=\left(\mathbf{p}_{\mathbf{2}}, \mathbf{p}_{\mathbf{3}}, \mathbf{p}_{\mathbf{4}}, \ldots, \mathbf{p}_{\mathbf{1}}, p_{t+1}, p_{t+2}, \ldots, p_{|A| !}\right)
$$

From ballot neutrality, we know that $\varphi(P)=\varphi\left(P^{i}\right)$ for any $i \in\{1, \ldots, t-$ $1\}$. By design, merging all these profiles $\left(P, P^{1}, P^{2}, \ldots, P^{t-1}\right)$ gives us $t P^{\prime}$, and from consistency, we get that $\varphi\left(t P^{\prime}\right)=\varphi(P)$. From Lemma 2.2, $\varphi(P)=\varphi\left(t P^{\prime}\right)=\varphi\left(P^{\prime}\right)$ is guaranteed.

ii) Let $P$ and $p=\left(p_{1}, p_{2}, \ldots, p_{|A| !}\right)$ be as in the Lemma with $|\varphi(P)|=t$ and $P^{\prime \prime}$ as defined in the Lemma. Consider the following profiles with the same enumeration on $\mathcal{L}(A)$ where $p_{t+1}, p_{t+2}, \ldots, p_{|A|}$ ! rotates and bold numbers indicate the support for the chosen delegates:

$$
\begin{gathered}
P^{1} \in \mathcal{L}(A)^{n} \text { such that } p^{1}=\left(\mathbf{p}_{\mathbf{1}}, \mathbf{p}_{\mathbf{2}}, \ldots, \mathbf{p}_{\mathbf{t}}, p_{|A| !}, p_{t+1}, p_{t+2}, \ldots, p_{|A| !-1}\right) \\
P^{2} \in \mathcal{L}(A)^{n} \text { such that } p^{2}=\left(\mathbf{p}_{\mathbf{1}}, \mathbf{p}_{\mathbf{2}}, \ldots, \mathbf{p}_{\mathbf{t}}, p_{|A| !-2}, p_{|A| !}, p_{t+1}, \ldots, p_{|A| !-2}\right)
\end{gathered}
$$

$P^{t-1} \in \mathcal{L}(A)^{n}$ such that $p^{t-1}=\left(\mathbf{p}_{\mathbf{1}}, \mathbf{p}_{\mathbf{2}}, \ldots, \mathbf{p}_{\mathbf{t}}, p_{t+2}, p_{t+3}, p_{t+4}, \ldots, p_{t+1}\right)$ 
From ballot neutrality, we know that $\varphi(P)=\varphi\left(P^{i}\right)$ for any $i \in\{1, \ldots, t-$ $1\}$. By design, merging all these profiles $\left(P, P^{1}, P^{2}, \ldots, P^{t-1}\right)$ gives us $t P^{\prime \prime}$, and from consistency we get that $\varphi\left(t P^{\prime \prime}\right)=\varphi(P)$. From Lemma 2.2, $\varphi(P)=\varphi\left(t P^{\prime \prime}\right)=\varphi\left(P^{\prime \prime}\right)$ is guaranteed.

\section{A.2.6 Proof of Lemma 2.4}

Lemma 2.4. If a rule $\varphi$ satisfies ballot neutrality and strategy-proofness, then for all $A \subsetneq \mathcal{A}, N \subsetneq \mathcal{N}$, and $P \in \mathcal{L}(A)^{n}$ if $R \in \varphi(P)$, then we have

$$
p(R)>\sum_{R^{\prime} \notin \varphi(P)} p\left(R^{\prime}\right) .
$$

Proof:. Let us denote by $W=\varphi(P)$ the preferences of "winning" agents, and by $L=R P(P) \backslash \varphi(P)$ the preferences of "losing" agents, where $R P(P)=$ $\{R \in \mathcal{L}(A) \mid p(R)>0\}$ is again the set of reported preferences. Suppose, for a contradiction, that there exists a profile $P \in \mathcal{L}(A)^{n}$ and a preference $R_{t} \in \varphi(P)$ such that:

$$
p\left(R_{t}\right) \leq \sum_{\bar{R} \in L} p(\bar{R})
$$

That is, a preference $R_{t}$ in the delegation has weakly less support than the total support for all preferences of losing agents combined. By Remark 2.1, there exists an expansion of $P$, in which the corresponding injections of $W$ and $L$ are clustered far away from each other. By the same logic, one can find an expansion by $\pi$, say $\bar{P}$, if needed to an even larger alternative set, in which in addition to having $\pi(W)$ and $\pi(L)$ as far away clusters, $R_{\pi(t)}$ is relatively closer to $\pi(L)$ than to the other preferences in $\pi(W)$. Formally:

$$
\max _{R, R^{\prime} \in \pi(L)} \delta\left(R, R^{\prime}\right)<\min _{R \in \pi(L), R^{\prime} \in \pi(W)} \delta\left(R, R^{\prime}\right)
$$

and for any $R, R^{\prime} \in \pi(L), \tilde{R} \in \pi(W)$,

$$
\delta\left(R, R^{\prime}\right)<\delta\left(R, R_{\pi(t)}\right)<\delta(R, \tilde{R})
$$


Now consider a transformation of this expansion, denoted by $\bar{P}^{\prime}$, where all losing agents concentrate on a preference of a fellow losing agent, say $R^{s}$. By construction:

$$
\sum_{R \in L} p(R)=\bar{p}^{\prime}\left(R^{s}\right)
$$

By supposition, $\bar{p}\left(R_{\pi(t)}\right) \leq \bar{p}\left(R^{s}\right)$. Note that by coalitional strategyproofness, we have $R^{s} \notin \varphi\left(\bar{P}^{\prime}\right)$. Then we have two cases:

Case 1: If $R_{\pi(t)} \in \varphi\left(\bar{P}^{\prime}\right)$, then by Lemma $2.1, R^{s}$ should also be in the delegation $\varphi\left(\bar{P}^{\prime}\right)$, which is a contradiction.

Case 2: If $R_{\pi(t)} \notin \varphi\left(\bar{P}^{\prime}\right)$, furthermore by (A.7), $R_{\pi(t)}$ is a favorable preference for all agents with $\bar{P}(i) \in \pi(L)$. Then the agents in $L$ can misreport (and disperse back to their preferences in $\bar{P})$. As $R_{\pi(t)} \in \varphi(\bar{P})$, this contradicts strategy-proofness.

\section{A.2.7 Proof of Lemma 2.5}

Lemma 2.5. If a rule $\varphi$ satisfies Pareto optimality, consistency, ballot neutrality, and strategy-proofness, then for all $A \subsetneq \mathcal{A}$, and $N \subsetneq \mathcal{N}$ the corresponding vector satisfies that $k_{t}^{\varphi}(A) \geq \frac{k_{t-1}^{\varphi}(A)+1}{2}$ for all $t \in\{2,3, \ldots,|A| !\}$.

Proof:. Let us start with some $A \subsetneq \mathcal{A}$ and some $t<|A|$ !-1. Using consistency, we can pick some $N \subsetneq \mathcal{N}$ with $|N|=n$ divisible by all numbers up to $t+1$ without loss of generality. Let us take a specific $P \in \mathcal{L}(A)^{n}$, which is defined as $p_{i}=a$ for all $i \in\{1,2, \ldots, t+1\}$ and $p_{i}=0$ for $i \in\{t+2, t+3, \ldots,|A| !\}$. From Proposition 2.1 and ballot neutrality, we know that the delegation includes only the first $t+1$ preferences. Let us denote this profile as (with bold numbers indicating the support for the chosen delegates)

$$
p=(\underbrace{\mathbf{a}, \mathbf{a}, \ldots, \mathbf{a}}_{t+1 \text { times }}, \underbrace{0,0, \ldots, 0}_{|A| !-t-1 \text { times }}) .
$$

Now, let us deal with a modification $P^{\prime} \in \mathcal{L}(A)^{n}$ for this profile which is defined as $p_{i}^{\prime}=a^{\prime}=\frac{n k_{t}}{t}$ for all $i \in\{1,2, \ldots, t\}, p_{t+1}^{\prime}=b=n\left(1-k_{t}\right)$ and $p_{i}^{\prime}=0$ for $i \in\{t+2, t+3, \ldots,|A| !\}$. Since the first $t$ preferences have $k_{t}$ support 
in total, by definition of $k_{t}$ and by Lemma 2.3, we know that the delegation includes only the first $t$ preferences. Let us denote this profile as

$$
p^{\prime}=(\underbrace{\mathbf{a}^{\prime}, \mathbf{a}^{\prime}, \ldots, \mathbf{a}^{\prime}}_{t \text { times }}, b, \underbrace{0,0, \ldots, 0}_{|A| !-t-1 \text { times }}) .
$$

Another relevant modification of this profile, $P^{\prime \prime} \in \mathcal{L}(A)^{n}$ will be defined as $p_{i}^{\prime \prime}=a^{\prime \prime}=\frac{n-2 b}{t-1}$ for all $i \in\{1,2, \ldots, t\}, p_{i}^{\prime \prime}=b=n\left(1-k_{t}\right)$ for $i \in\{t, t+1\}$ and $p_{i}^{\prime \prime}=0$ for $i \in\{t+2, t+3, \ldots,|A| !\}$, e.g. $p^{\prime \prime}=\left(a^{\prime \prime}, a^{\prime \prime}, \ldots, a^{\prime \prime}, b, b, 0,0, \ldots, 0\right)$. By Proposition 2.1 we have that $\varphi\left(P^{\prime \prime}\right) \subseteq R P(P)$, and by ballot neutrality either i) $\varphi\left(P^{\prime \prime}\right)=\left\{R_{1}, R_{2}, \ldots, R_{t+1}\right\}$ or ii) $\varphi\left(P^{\prime \prime}\right)=\left\{R_{1}, R_{2}, \ldots, R_{t-1}\right\}$. Suppose, for a contradiction, that the former is the case. Let us take the average of support for the first $t$ preferences to get $P^{\prime}$. By Remark 2.3, this should not change the delegation. However, $\varphi\left(P^{\prime}\right)=\left\{R_{1}, R_{2}, \ldots, R_{t}\right\} \neq\left\{R_{1}, R_{2}, \ldots, R_{t+1}\right\}=$ $\varphi\left(P^{\prime \prime}\right)$, which is a contradiction. So, it must be that ii) is the case, $\varphi\left(P^{\prime \prime}\right)=$ $\left\{R_{1}, R_{2}, \ldots, R_{t-1}\right\}$. Let us denote this profile as

$$
p^{\prime \prime}=(\underbrace{\mathbf{a}^{\prime \prime}, \mathbf{a}^{\prime \prime}, \ldots, \mathbf{a}^{\prime \prime}}_{t-1 \text { times }}, b, b, \underbrace{0,0, \ldots, 0}_{|A| !-t-1 \text { times }}) .
$$

By definition, $k_{t-1}$ is the minimal support for all delegations with size $t-1$. Since only the first $t-1$ preferences are in the delegation, total support for the first $t-1$ preferences could be at least $k_{t-1}$. Then, $(t-1) a^{\prime \prime}=(t-1) \frac{n-2 b}{t-1}=$ $n\left(1-2\left(1-k_{t}\right)\right) \geq n k_{t-1}$. After rearranging we get that

$$
k_{t} \geq \frac{k_{t-1}+1}{2}
$$

\section{A.2.8 Proof of Lemma 2.6}

Lemma 2.6. If a rule $\varphi$ satisfies Pareto optimality, consistency, ballot neutrality, and strategy-proofness, then for all $A \subsetneq \mathcal{A}, N \subsetneq \mathcal{N}$ and for any $P \in \mathcal{L}(A)^{n}$ such that $p_{1} \geq n k_{1}^{\varphi}(A)$, we have that $\varphi(P)=\left\{R_{1}\right\}$.

Proof:. Let $P^{*} \in \mathcal{L}(A)^{n^{*}}$ be one of the profiles where $\left|\varphi\left(P^{*}\right)\right|=1$ and $p_{1}^{*}=n^{*} k_{1}^{\varphi}(A)$, i.e., one of the profiles wherein only a single delegate is assigned 
whose relative support defines $k_{1}$ in the corresponding vector. Consider now any $P \in \mathcal{L}(A)^{n}$ with $p_{1} \geq n k_{1}^{\varphi}(A)$. By Lemma $2.1, R_{1} \in \varphi(P)$ and by ballot neutrality we can assume that $\varphi\left(P^{*}\right)=\left\{R_{1}\right\}$, i.e., the strongest ranking is the same both in $P$ and $P^{*}$. Next, we show that $R_{1}$ is the only delegate assigned to $P$, i.e., $\left\{R_{1}\right\}=\varphi(P)$.

By consistency, we can replicate profiles $P$ and $P^{*}\left(n^{*}\right.$ and $n$ times respectively) with no changes in the delegation. With abuse of notation, let us denote these replicated profiles by $P, P^{*} \in \mathcal{L}(A)^{n \times n^{*}}$. So, we have $p_{1}^{*}=n n^{*} k_{1}^{\varphi}(A)$ and $p_{1} \geq n n^{*} k_{1}^{\varphi}(A)$.

Suppose, for a contradiction, that $\varphi(P) \supsetneq\left\{R_{1}\right\}$, so there is another delegate, say $R_{k}$ in the delegation. Let us partition $\mathcal{L}(A)$ into two sets, $X=\mathcal{L}(A) \backslash\left\{R_{1}\right\}$ and $Y=\left\{R_{1}\right\}$. By Remark 2.1, there exists an expansion of $P$ by $\pi$, say $\bar{P}$, in which the injection of $X$, i.e., $\pi(X)$ is clustered far away from the injection of $R_{1}$, i.e., $R_{\pi(1)}$. Formally:

$$
\max _{R, R^{\prime} \in \pi(X)} \delta\left(R, R^{\prime}\right)<\min _{R \in \pi(X)} \delta\left(R, R_{\pi(1)}\right)
$$

Note that $R_{\pi(1)}=\pi(Y)$ and $R_{\pi(k)} \in \pi(X)$. Let $\bar{P}^{*}$ denote the expansion of $P^{*}$ by the same injection, $\pi$. By ballot neutrality, i) $R_{\pi(k)} \notin \varphi\left(\bar{P}^{*}\right)$, implying $k^{t h}$ strongest preference of $\bar{P}^{*}$ is not in the delegation of $\bar{P}^{*}$, and ii) $R_{\pi(k)} \in \varphi(\bar{P})$, implying that the $k^{\text {th }}$ strongest preference of $\bar{P}$ is in the delegation of $\bar{P}$. Note that as $\bar{p}_{1}^{*} \leq \bar{p}_{1}$, from $\bar{P}^{*}$ to $\bar{P}$ this means that there is a coalition of agents moving from $\pi(X)$ to $R_{\pi(1)}$, resulting in $R_{\pi}(k) \in \varphi(\bar{P})$. As $R_{\pi(k)} \in \pi(X)$, by (A.8), this contradicts coalitional strategy-proofness. Hence $R_{\pi(k)} \notin \varphi(\bar{P})$. Ballot neutrality then implies that $R_{k} \notin \varphi(P)$.

\section{A.2.9 Proof of Lemma 2.7}

Lemma 2.7. If a rule $\varphi$ satisfies Pareto optimality, consistency, ballot neutrality, and strategy-proofness, then for all $A \subsetneq \mathcal{A}, N \subsetneq \mathcal{N}$ and for any $P \in \mathcal{L}(A)^{n}$ such that

i) for some $t>1, \sum_{i=1}^{t} p_{i} \geq n k_{t}^{\varphi}(A)$ and, 
ii) for all $l<t, \sum_{i=1}^{l} p_{i}<n k_{l}^{\varphi}(A)$

we have: $\varphi(P)=\left\{R_{1}, R_{2}, \ldots, R_{t}\right\}$.

Proof:. Take any $P \in \mathcal{L}(A)^{n}$ as defined in the lemma. As $\sum_{i=1}^{l} p_{i}<n k_{l}$ for all $l<t$, by definiton of the corresponding vector, we have $|\varphi(P)| \neq l$. This means that $|\varphi(P)| \geq t$. By Lemma 2.1 we get that $\varphi(P) \supseteq\left\{R_{1}, R_{2}, \ldots, R_{t}\right\}$. Next, we show that $\varphi(P)=\left\{R_{1}, R_{2}, \ldots, R_{t}\right\}$.

By consistency, we can assume that $\sum_{i=1}^{t} p_{i}$ is divisible by $t$ without loss of generality. By Remark 2.3, we can take the average support for the first $t$ preferences without changing the delegation. Let us denote this modified profile by $P^{\prime}$, which is defined as $p_{i}^{\prime}=a^{\prime}=\sum_{l=1}^{t} \frac{p_{l}}{t}$ if $i \leq t$ and $p_{i}^{\prime}=p_{i}$ if $i>t$.

Let $P^{*} \in \mathcal{L}(A)^{n^{*}}$ be one of the profiles where $\left|\varphi\left(P^{*}\right)\right|=t, \sum_{i=1}^{t} p_{i}^{*}=n^{*} k_{t}$, i.e., one of the profiles wherein only the strongest $t$ delegates are assigned whose relative total support defines $k_{t}$ in the corresponding vector.

By consistency, we can assume that $\sum_{i=1}^{t} p_{i}^{*}$ is divisible by $t$ without loss of generality. By Remark 2.3, we can take the average support for the first $t$ preferences without changing the delegation. Let us denote this modified profile also by $P^{*}$, where $p_{i}^{*}=a=\frac{n^{*} k_{t}}{t}$ for all $i \leq t$.

Using consistency, we can replicate profiles $P^{\prime}$ and $P^{*}\left(n^{*}\right.$ and $n$ times respectively) with no changes in the delegation. With abuse of notation, let us denote these replicated profiles by $P^{\prime}, P^{*} \in \mathcal{L}(A)^{n \times n^{*}}$. By construction, the total support for the strongest $t$ preferences in $P^{\prime}$ is larger than those in $P^{*}$, i.e., $n^{*} a^{\prime} t \geq$ nat.

Suppose, for a contradiction, that $\varphi\left(P^{\prime}\right) \supsetneq\left\{R_{1}, R_{2}, \ldots, R_{t}\right\}$ so there is another delegate, say $R_{k}$ with $k>t$ in the delegation. Let us partition $\mathcal{L}(A)$ into two sets, $X=\mathcal{L}(A) \backslash\left\{R_{1}, R_{2}, \ldots, R_{t}\right\}$ and $Y=\left\{R_{1}, R_{2}, \ldots, R_{t}\right\}$. By Remark 2.1, there exists an expansion of $P^{\prime}$ by $\pi$, say $\bar{P}^{\prime}$, in which the injection of $Y$ is clustered far away from the injection of $X$. Formally:

$$
\max _{R, R^{\prime} \in \pi(X)} \delta\left(R, R^{\prime}\right)<\min _{R \in \pi(X), R^{\prime} \in \pi(Y)} \delta\left(R, R^{\prime}\right)
$$


Note that $\left\{R_{\pi(1)}, R_{\pi(2)}, \ldots, R_{\pi(t)}\right\}=\pi(Y)$ and $R_{\pi(k)} \in \pi(X)$. Let $\bar{P}^{*}$ denote the expansion of $P^{*}$ by the same injection, $\pi$. By ballot neutrality, $R_{\pi(k)} \notin \varphi\left(\bar{P}^{*}\right)$ while $R_{\pi(k)} \in \varphi\left(\bar{P}^{\prime}\right)$. Note that from $\bar{P}^{*}$ to $\bar{P}^{\prime}$ there is a coalition of agents moving from $\pi(X)$ to $\pi(Y)$, resulting in $R_{\pi}(k) \in \varphi\left(\bar{P}^{\prime}\right)$. As $R_{\pi(k)} \in \pi(X)$, by (A.9), this contradicts coalitional strategy-proofness.

\section{A.2.10 Proof of Lemma 2.8}

Lemma 2.8. If a rule $\varphi$ satisfies ballot neutrality, then for any $A \subsetneq \bar{A} \subsetneq \mathcal{A}$, the corresponding vector satisfies that $k^{\varphi}(A)_{t}=k^{\varphi}(\bar{A})_{t}$ for all $t \in\{1,2, \ldots,|A| !\}$.

Proof:. We denote corresponding threshold vectors as

$$
\begin{aligned}
k^{\varphi}(A) & =\left[k_{1}, k_{2}, \ldots, k_{|A| !}\right], \\
k^{\varphi}(\bar{A}) & =\left[k_{1}^{\prime}, k_{2}^{\prime}, \ldots, k_{|\bar{A}| !}^{\prime}\right] .
\end{aligned}
$$

Suppose, for a contradiction, that there exist $A, \bar{A} \subsetneq \mathcal{A}$ such that $A \subsetneq \bar{A}$ with for some $t \in\{1,2, \ldots,|A| !\}, k_{t} \neq k_{t}^{\prime}$ while for all $i \in\{1,2, \ldots, t-1\}$, $k_{i}=k_{i}^{\prime}$. Without loss of generality, assume that $k_{t}<k_{t}^{\prime}$.

Then we construct a profile $P \in \mathcal{L}(A)^{n}$ and an expansion of $P$, by some $\pi$, denoted by $\bar{P}$ such that $\rho_{t}>k_{t}, \rho_{t}<k_{t}^{\prime}$ and $\rho_{i}<k_{i}$ for all $i \in\{1,2, \ldots, t-1\}$.

By Lemma 2.6 and Lemma 2.7 we have that $\varphi(P)=\left\{R_{1}, R_{2}, \ldots, R_{t}\right\}$, while $\varphi\left(P^{\prime}\right) \supsetneq\left\{R_{\pi(1)}, R_{\pi(2), \ldots, R_{\pi(t)}}\right\}$. Since $\varphi\left(P^{\prime}\right)$ includes other elements than the image of $\varphi(P)$ under $\pi$, this contradicts ballot neutrality.

\section{A.3 Independence of the conditions}

The conditions used in the characterization were: Pareto optimality, consistency, strategy-proofness, and ballot neutrality. Below, to put forward the logical independence of those, let us take a look at the following four social welfare correspondences.

- All but Pareto optimality: $\varphi(P)=\mathcal{L}(A)$ for any $P \in \mathcal{L}(A)^{n}$. 
- All but consistency:

$$
\varphi(P)= \begin{cases}R, & \text { if } n \text { is odd and } \exists R \text { with } p(R)>\frac{N}{2} \\ R, & \text { if } n \text { is even and } \exists R \text { with } p(R)>\frac{2 N}{3} \\ R P(P), & \text { otherwise. }\end{cases}
$$

- All but strategy-proofness: $\varphi(P)=\left\{R \mid p(R) \geq p\left(R^{\prime}\right)\right.$ for all $R^{\prime} \in$ $\mathcal{L}(A)\}$.

- All but ballot neutrality:

$$
\varphi(P)= \begin{cases}\mathcal{L}(A), & \text { if }|\mathcal{L}(A) \backslash R P(P)|=1 \\ R P(P), & \text { otherwise. }\end{cases}
$$




\section{Appendix B}

\section{Appendix for Chapter 3}

\section{B.1 Proof of Proposition 3.5}

Proposition 3.5. There exists no social welfare rule which satisfies $P C W$ and $W C C W$.

Proof:. Suppose for a contradiction $\varphi$ is a rule which satisfies PCW and WCCW.

The following example is directly from Moulin (1988). Note that, in the original proof 25 agents was used at most, while here we use 27 . Let us denote the profile we will work on by $p$ :

\begin{tabular}{cccc}
3 & 3 & 5 & 4 \\
\hline \hline$a$ & $a$ & $d$ & $b$ \\
$d$ & $d$ & $b$ & $c$ \\
$c$ & $b$ & $c$ & $a$ \\
$b$ & $c$ & $a$ & $d$ \\
\hline
\end{tabular}

- Let us assume 6 agents with bdac joins the election.

After their arrival, $d b a c$ is the Condorcet Ranking. From $P C W, b d \notin$ $\bigcup_{R \in \varphi(p)} R .^{1}$

\footnotetext{
${ }^{1}$ Note that since 6 agents with $b d a c$ can not make $b d$ included in all of the outcomes, from
} 
- Let us assume 8 agents with cbad joins the election instead.

After their arrival, bcad is the Condorcet Ranking. From $P C W, c b \notin$ $\bigcup_{R \in \varphi(p)} R$

- Let us assume 4 agents with $d a b c$ joins the election instead.

After their arrival, $a d b c$ is the Condorcet Ranking. From $P C W, d a \notin$ $\bigcup_{R \in \varphi(p)} R$.

So any rule which is $P C W$ and $W C C W$ should have $\{a d, d b, b c\} \in \bigcap_{R \in \varphi(p)} R$. More exactly: $\varphi(p)=\{a d b c\}$.

Again, following from Moulin (1988), we will be adding 4 agents with cabd to original profile. Let us denote this profile with $p^{\prime}$.

\begin{tabular}{ccccc}
3 & 3 & 5 & 4 & 4 \\
\hline \hline$a$ & $a$ & $d$ & $b$ & $c$ \\
$d$ & $d$ & $b$ & $c$ & $a$ \\
$c$ & $b$ & $c$ & $a$ & $b$ \\
$b$ & $c$ & $a$ & $d$ & $d$ \\
\hline
\end{tabular}

- Let us assume 8 agents with $d a b c$ joins the election.

After their arrival, $a d b c$ is the Condorcet Ranking. From $P C W, d a \notin$ $\bigcup_{R \in \varphi\left(p^{\prime}\right)} R$.

- Let us assume 4 agents with cbad joins the election instead.

After their arrival, bcad is the Condorcet Ranking. From $P C W, c b \notin$ $\bigcup_{R \in \varphi\left(p^{\prime}\right)} R$.

- Let us assume 6 agents with $a c d b$ joins the election instead.

After their arrival, $c a d b$ is the Condorcet Ranking. From $P C W, a c \notin$ $\bigcup_{R \in \varphi\left(p^{\prime}\right)} R$

$P C W, b d$ can not be in any of the outcomes with one less such agent as well. A similar argument holds between 5 and 4,4 and 3 and so on to ensure $b d \notin \bigcup_{\varphi(p)} R$. In the following parts of the proof this feature of $P C W$ will be repetitively used. 
So any rule which is $P C W$ and $W C C W$ should have $\{b c, c a, a d\} \in \bigcap_{R \in \varphi\left(p^{\prime}\right)} R$. More exactly: $\varphi\left(p^{\prime}\right)=\{b c a d\}$.

Let us remind that the only difference between $p$ and $p^{\prime}$ was addition of 4 agents with $c a b d$. Those 4 agents had lost $a b$ by attending, $(c a b d \cap a d b c \nsubseteq \nsubseteq b c a d)$ which contradicts $P C W$.

\section{B.2 Proofs for Copeland rule}

\section{B.2.1 Copeland does not satisfy $W P C W$ and $P C W$.}

Proof of Proposition 3.6. Consider the following profile $p$ with twenty-one individuals and the set of alternatives $A=\{a, b, c, d\}$.

\begin{tabular}{ccccc}
3 & 3 & 4 & 5 & 6 \\
\hline \hline$a$ & $a$ & $b$ & $d$ & $b$ \\
$d$ & $d$ & $c$ & $b$ & $d$ \\
$b$ & $c$ & $a$ & $c$ & $a$ \\
$c$ & $b$ & $d$ & $a$ & $c$ \\
\hline
\end{tabular}

It can be verified that $T_{p}=\{d a, d b, d c, b a, b c, a c\}$ hence $\varphi^{\text {Copeland }}(p)=$ $\{d b a c\}$. Now assume the agent $i$ with the preference ranking $p(i)=b d a c$, decides not to participate. Then it can be verified that $T_{p_{-i}}=\{d b, d c, b a, b c, a c\}$ and therefore $\varphi^{\text {Copeland }}\left(p_{-i}\right)=\{b d a c, d b a c\}$. As bdac maximizes the utility of individual $i$ and $U_{i}(b d a c)>U_{i}(d b a c)$, it follows that Copeland does not satisfy $W P C W$.

\section{B.2.2 Copeland satisfies $W C C W$, but it does not satisfy $C C W$.}

\section{Proof of Proposition 3.7.}

WCCW is satisfied:

Let $p$ be a profile and let $R=a_{1} a_{2} \ldots a_{m}$ be the Condorcet ranking of the profile. By definition of Condorcet ranking $n_{a_{i} a_{k}}>0$ for all $i \in\{1, \cdots, m\}$ 
and $k \in\{i+1, \cdots, m\}$. Since $\operatorname{Cscore}\left(a_{i}, p\right)=\left|\left\{k \in A: a_{i} a_{k} \in T_{p}\right\}\right|=$ $\left|\left\{a_{i+1}, a_{i+2}, \cdots, a_{m}\right\}\right|=m-i$, then by definition of Copeland rule $a_{1} a_{2} \cdots a_{m}$ will be the outcome which coincides with the Condorcet ranking.

\section{CCW is not satisfied:}

Consider the profile from Appendix B.2.1, where agent $i$ leaves. Remaining profile with twenty individuals and the set of alternatives $A=\{a, b, c, d\}$ will be denoted by $p^{\prime}$.

\begin{tabular}{lllll}
3 & 3 & 4 & 5 & 5 \\
\hline \hline$a$ & $a$ & $b$ & $d$ & $b$ \\
$d$ & $d$ & $c$ & $b$ & $d$ \\
$b$ & $c$ & $a$ & $c$ & $a$ \\
$c$ & $b$ & $d$ & $a$ & $c$ \\
\hline
\end{tabular}

It can be verified that $T_{p^{\prime}}=\{d b, d c, b a, b c, a c\}$ and therefore $\varphi^{\text {Copeland }}\left(p^{\prime}\right)=$ $\{b d a c, d b a c\}$. Note that $\cdots b d \cdots \in \varphi\left(p^{\prime}\right)$ even though $n_{d b}>0$, which contra$\operatorname{dicts} C C W$.

\section{B.3 Proofs for Minimax rule}

\section{B.3.1 Minimax does not satisfy $W P C W$ and $P C W$.}

Proof of Proposition 3.8. Consider the following profile $p$ with seventeen individuals and the set of alternatives $A=\{a, b, c, d\}$.

\begin{tabular}{cccccccc}
3 & 2 & 2 & 2 & 2 & 2 & 2 & 2 \\
\hline \hline$a$ & $d$ & $a$ & $c$ & $b$ & $c$ & $d$ & $b$ \\
$b$ & $c$ & $d$ & $b$ & $d$ & $a$ & $c$ & $a$ \\
$c$ & $a$ & $b$ & $a$ & $a$ & $b$ & $a$ & $d$ \\
$d$ & $b$ & $c$ & $d$ & $c$ & $d$ & $b$ & $c$ \\
\hline
\end{tabular}


The above profile can be summarized in the following "profile matrix" $\bar{p}$ where the cell $x y$ in $\bar{p}$ denotes the number of individuals that prefer $x$ to $y$.

$$
\bar{p}=\begin{gathered}
a \\
b \\
c \\
d \\
d
\end{gathered}\left[\begin{array}{cccc}
0 & 11 & 9 & 11 \\
6 & 0 & 9 & 11 \\
8 & 8 & 0 & 7 \\
6 & 6 & 10 & 0
\end{array}\right]
$$

It can be verified that $\varphi^{\operatorname{Minimax}}(p)=\{a c b d, a c d b\}$. Now assume the agent $i$ with the preference ranking $p(i)=a b c d$ decides not to participate. Then it can be verified that the profile matrix would be as the following,

$$
\bar{p}_{-i}=\begin{gathered}
a \\
b \\
c \\
d
\end{gathered}\left[\begin{array}{cccc}
0 & b & c & d \\
6 & 0 & 8 & 10 \\
8 & 8 & 0 & 6 \\
6 & 6 & 10 & 0
\end{array}\right]
$$

It can be verified that $\varphi^{\text {Minimax }}\left(p_{-i}\right)=\{a b c d, a b d c, a c b d, a c d b, a d b c, a d c b\}$. It is easy to see that acbd $\in \underset{R \in \varphi^{\text {Minimax }}(p)}{\arg \max } U_{i}(R)$ and abcd $\in$ $\underset{R \in \varphi^{\text {Minimax }}\left(p_{-i}\right)}{\arg \max } U_{i}(R)$. Since $U_{i}(a b c d)>U_{i}(a c b d)$, it follows that Minimax does not satisfy $W P C W$.

\section{B.3.2 Minimax does not satisfy $W C C W$ and $C C W$.}

Proof of Proposition 3.9. Let $A=\{a, b, c\}$ and $p=(a b c, a b c, a b c, c a b, c b a)$ be a profile. It is obvious that the Condorcet ranking is $a b c$. It can be verified that $M \operatorname{score}(a, p)=2, \operatorname{Mscore}(b, p)=4$, and $\operatorname{Mscore}(c, p)=3$ hence $\varphi^{\text {Minimax }}(p)=\{a c b\}$. Therefore Minimax does not satisfy Condorcet criteria for welfare rules. 


\section{B.4 Proofs for Scoring rules}

\section{B.4.1 Scoring rules satisfy $P C W$ and $W P C W$.}

Proof of Proposition 3.10. Consider any profile $p \in \mathcal{L}^{N}$. Take any $a b \in p(i)$. If there exists $R$ such that $a b \in R$ with $R \in \varphi^{S C}\left(p_{-i}\right)$, then this means:

$$
\operatorname{Score}\left(a, p_{-i}\right) \geq \operatorname{Score}\left(b, p_{-i}\right) .
$$

Also since $a b \in p(i)$ we have,

$$
\operatorname{Score}(a, p(i))>\operatorname{Score}(b, p(i)) \text {. }
$$

By definition, Score $(a, p)=\operatorname{Score}\left(a, p_{-i}\right)+\operatorname{Score}(a, p(i))$. Then by (B.1) and (B.2), we have $\operatorname{Score}(a, p)>\operatorname{Score}(b, p)$ hence for all $R^{\prime} \in \varphi^{S C}(p), a b \in R^{\prime}$.

\section{B.4.2 Degenerate scoring rules satisfy $W P C W$, but they do not satisfy $P C W$}

\section{Proof of Proposition 3.11.}

WPCW is satisfied:

Consider any profile $p \in \mathcal{L}^{n}$. Let $\hat{R} \in \underset{R \in \varphi^{S C}(p-i)}{\arg \max } U_{i}(R)$ and $\bar{R} \in$ $\arg \max U_{i}(R)$ for some $i \in N$. $R \in \varphi^{S C}(p)$

We first show that $(p(i) \cap \hat{R}) \subseteq(p(i) \cap \bar{R})$. Take any distinct pair of alternatives $a b \in(\hat{R} \cap p(i))$. Since $a b \in \hat{R}$ we have,

$$
\operatorname{Score}\left(a, p_{-i}\right) \geq \operatorname{Score}\left(b, p_{-i}\right) .
$$

Also since $a b \in p(i)$ we have,

$$
\operatorname{Score}(a, p(i)) \geq \operatorname{Score}(b, p(i)) \text {. }
$$

By definition, Score $(a, p)=\operatorname{Score}\left(a, p_{-i}\right)+\operatorname{Score}(a, p(i))$. Then by (B.3) and (B.4), we have $\operatorname{Score}(a, p) \geq \operatorname{Score}(b, p)$ hence $a b \in \bar{R}$. Since this is true for any $a b \in p(i)$, we have $(p(i) \cap \hat{R}) \subseteq(p(i) \cap \bar{R})$ which implies,

$$
|p(i) \cap \hat{R}| \leq|p(i) \cap \bar{R}|
$$


Note that $\delta\left(R, R^{\prime}\right)=\left(m^{2}+m\right) / 2-\left|R \cap R^{\prime}\right|$ for any $R, R \in \mathcal{L}(A)$. Using this,

$$
\begin{aligned}
& \delta(p(i), \hat{R})=\left(\frac{m^{2}+m}{2}-|p(i) \cap \hat{R}|\right) \\
& \delta(p(i), \bar{R})=\left(\frac{m^{2}+m}{2}-|p(i) \cap \bar{R}|\right)
\end{aligned}
$$

(B.5), together with the two equations above, yields $\delta(p(i), \bar{R}) \leq \delta(p(i), \hat{R})$.

\section{PCW is not satisfied:}

Take any score vector $s=\left(s_{1}, \cdots, s_{m}\right)$ with some $s_{k}=s_{l}$ for $k, l \in$ $\{1, \cdots, m\}$ and $k<l$. Consider two alternatives $a, b \in A$ and a profile $p \in \mathcal{L}^{2 n}$. Let us give the structure of the profile as the following. For any $i \in\{1, \cdots, n\}$, $p(i)=R$ and for any $j \in\{n+1, \cdots, 2 n\}, p(j)=\{a b \in A \times A \mid b a \in R\}$. Note that, for any two $a, b \in A, \operatorname{Score}(a, p)=\operatorname{Score}(b, p)$, so for any $a b \in A \times A$, there exists a $R \in \varphi^{S C}(p)$ such that $a b \in R$.

Now, let us add a new agent with preference $p(2 n+1)$. In $p(2 n+1)$, let us denote the alternative at $k^{\text {th }}$ place by $c$ and the alternative at $l^{\text {th }}$ by $d$. Obviously, $c d \in p(2 n+1)$. After participation by this agent, let us denote this new profile by $p^{\prime} \in \mathcal{L}^{2 n+1}$. From the score vector, we know that $\operatorname{Score}\left(c, p^{\prime}\right)=\operatorname{Score}\left(d, p^{\prime}\right)$, which implies there exists $R^{\prime}, R^{\prime \prime} \in \varphi^{S C}\left(p^{\prime}\right)$ such that $c d \in R^{\prime}$ and $d c \in R^{\prime \prime}$ which contradicts with $P C W$.

\section{B.4.3 Scoring rules do not satisfy $W C C W$ and $C C W$.}

\section{Proof of Proposition 3.12.}

The following example is due to Condorcet (1788). Consider the following profile $p$ with eighty one individuals and the set of alternatives $A=\{a, b, c\}$.

\begin{tabular}{rrrrrr}
30 & 1 & 29 & 10 & 10 & 1 \\
\hline \hline$a$ & $a$ & $b$ & $b$ & $c$ & $c$ \\
$b$ & $c$ & $a$ & $c$ & $a$ & $b$ \\
$c$ & $b$ & $c$ & $a$ & $b$ & $a$ \\
\hline
\end{tabular}

It is straightforward to see that $a b c$ is the Condorcet ranking. As $\operatorname{Score}(a, p)=31 s_{1}+29 s_{2}+11 s_{3}, \operatorname{Score}(b, p)=39 s_{1}+31 s_{2}+11 s_{3}$, for any $s_{1} \geq s_{2}$, we have $\operatorname{Score}(b, p) \geq \operatorname{Score}(a, p)$. This results in $b a \in \varphi^{S C}(p)$, which contradicts $W C C W$. 


\section{B.5 Proofs for the Kemeny rule}

\section{B.5.1 Kemeny satisfies $W P C W$, but it does not satisfy $P C W$}

\section{Proof.}

\section{WPCW is satisfied:}

Let $p$ be a profile. Let $\bar{R} \in \underset{R \in \varphi^{\text {Kemeny }}(p)}{\arg \max } U_{i}(R)$ and $\hat{R} \in$ $\underset{R \in \varphi^{K e m e n y}\left(p_{-i}\right)}{\arg \max } U_{i}(R)$ for any agent $i$ in the set of individuals. Since $\bar{R} \in$ $\varphi^{\text {Kemeny }}(p)$, by definition of the Kemeny rule, $\sum_{i \in N} \delta(p(i), \bar{R}) \leq \sum_{i \in N} \delta(p(i), \hat{R})$, hence

$$
\sum_{j \in N \backslash\{i\}} \delta(p(j), \bar{R})+\delta(p(i), \bar{R}) \leq \sum_{j \in N \backslash\{i\}} \delta(p(j), \hat{R})+\delta(p(i), \hat{R})
$$

Since $\hat{R}=\varphi^{\text {Kemeny }}\left(p_{-i}\right)$, by definition of the Kemeny rule,

$$
\sum_{j \in N \backslash\{i\}} \delta\left(p_{-i}(j), \hat{R}\right) \leq \sum_{j \in N \backslash\{i\}} \delta\left(p_{-i}(j), \bar{R}\right) .
$$

As $p(j)=p_{-i}(j)$ for all $j \in N \backslash\{i\}$, then above equation is equivalent to,

$$
\sum_{j \in N \backslash\{i\}} \delta(p(j), \hat{R}) \leq \sum_{j \in N \backslash\{i\}} \delta(p(j), \bar{R}) .
$$

Adding $\delta(p(i), \hat{R})$ to both sides of (B.7) results in,

$$
\sum_{j \in N \backslash\{i\}} \delta(p(j), \hat{R})+\delta(p(i), \hat{R}) \leq \sum_{j \in N \backslash\{i\}} \delta(p(j), \bar{R})+\delta(p(i), \hat{R}) .
$$

Combining (B.6) and (B.8) results in,

$$
\sum_{j \in N \backslash\{i\}} \delta(p(j), \bar{R})+\delta(p(i), \bar{R}) \leq \sum_{j \in N \backslash\{i\}} \delta(p(j), \bar{R})+\delta(p(i), \hat{R}) .
$$

Therefore, $\delta(p(i), \bar{R}) \leq \delta(p(i), \hat{R})$ which completes the proof.

PCW is not satisfied: 
Let $A=\{a, b, c, d\}$ and $p=(a b c d, a b c d, d c b a, c d a b, d b c a)$ be a preference profile for five agents. It can be verified that $\varphi^{K}(p)=c d a b$ and $\varphi^{K}\left(p_{-5}\right)=$ $\{a b c d, a c b d, a c d b, c a b d, c a d b$, $c d a b\}$. Since $b c \in \bigcup_{R \in \varphi^{K}\left(p_{-5}\right)} R$ and $b c \notin \varphi(p)$, this contradicts $P C W$.

\section{B.5.2 Kemeny satisfies $C C W$ and $W C C W$.}

Proof. Theorem 3 of (Young and Levenglick, 1978) shows that the Kemeny rule satisfies $C C W$, which also implies $W C C W$.

\section{B.6 Proofs for Slater rule}

\section{B.6.1 Slater does not satisfy $W P C W$ and $P C W$.}

Proof. Consider the following profile $p$ with 10 agents that orders 4 alternatives:

\begin{tabular}{cccccccccc}
$p(1)$ & $p(2)$ & $p(3)$ & $p(4)$ & $p(5)$ & $p(6)$ & $p(7)$ & $p(8)$ & $p(9)$ & $p(10)$ \\
\hline$a$ & $d$ & $b$ & $d$ & $d$ & $c$ & $a$ & $d$ & $b$ & $c$ \\
$b$ & $c$ & $c$ & $a$ & $a$ & $b$ & $c$ & $b$ & $d$ & $a$ \\
$c$ & $a$ & $a$ & $b$ & $b$ & $d$ & $b$ & $a$ & $a$ & $b$ \\
$d$ & $b$ & $d$ & $c$ & $c$ & $a$ & $d$ & $c$ & $c$ & $d$ \\
\hline
\end{tabular}

Note that $T_{p}=\{a b, a c, d a, b c, b d\} \cdot \varphi^{S}(p)=\{a b c d, a b d c, b d a c, d a b c\}$. Consider a $11^{\text {th }}$ agent with $p(11)=c d a b$. Among the rankings in the outcome, dabc is the closest one, with $\delta(d a b c, c d a b)=3$. Let us consider the case when this agent joins the election. Denoting the new preference profile by $p^{\prime}$, note that $T_{p^{\prime}}=\{a b, a c, d a, b c, b d, c d\} \cdot \varphi^{S}\left(p^{\prime}\right)=\{a b c d\}$ with $\delta(a b c d, c d a b)=4$, which contradicts $W P C W$.

\section{B.6.2 Slater satisfies $C C W$ and $W C C W$.}

\section{Proof.}

Take any $p$, with $n_{a b}(p)>0$. Suppose for a contradiction that $R=\cdots b a \cdots$ is included in $\varphi^{S}(p)$. Let us define $\bar{R}=(R \backslash\{b a\}) \cup\{a b\}$, that is, $\bar{R}$ and 
$R$ are almost the same, with only $a$ and $b$ are reversed. Then we have: $\delta\left(\bar{R}, T_{p}\right)=\delta\left(R, T_{p}\right)-1$ which contradicts with $R \in \varphi^{S}(p)$. 


\section{Valorisation}

"What do you study, exactly?" is a question I heard many times, mostly from friends who are outside of the academia. While there is no easy answer to this question, I considered finding a close enough one is a challenge myself. In general, this thesis is in the field of social choice theory. Though, as soon as I mention the word "elections" everyone is so keen to finalize the explanation I started by guessing. "So, you collect election data and check some patterns and such?" I reply to those with saying some people also study things like that. But...

But what exactly I do? It is somehow easier to understand what does the political theorists do, what commentators do, what policy advisors do etc. Except the first one, they work with a given system, evaluate how things could be done better, using some existing tools to solve contemporary problems. In contrast to this, political theorists, philosophers try to understand the procedure. They not only define and observe the structure but also put things into a historical framework. By putting some distance between themselves and the reality, they are able to criticize and present alternatives. That is why I consider social choice theorists as a kind of philosophers.

While generally philosophers use history of thought, analogies between concepts and events, social choice theorists use the intricacies of mathematical objects to understand the underlying properties. While abstraction is always useful to seek alternatives, in this case, it already is included in the tool we use, mathematics. When it comes to elections, there are many criteria one would be interested in. It would be reasonable to assume that if there is an election 
system that encapsulates all, it has to be shown in a rigorous way. If there is not, this impossibility must be shown in a such a way that, there will not be any efforts wasted on the intersection of some ideals. Social choice is interested in both of these, most of its results are either characterizations which define things in the most concise way, or impossibilities that shows an incompatibility between different criteria.

As already discussed, this thesis in its total follows the discipline of social choice theory. So going over the chapters to summarize what has been done will be exemplifying the general picture. The first chapter is actually motivated by the civil war in Syria. Many of the proposed solutions to the crisis included gathering up a delegation to discuss what should be done, and how the different agents in Syrian society can compromise. This was all before most of the bloodshed, we hoped that this diplomatic procedure could at least ease the crisis a bit. While the events afterward proved us utterly wrong, our work tried to help this, or other procedures like this to pick a delegation in a neutral way, that is, it should be based on reasonable principles without implying any implicit advantage for any of the opinions to be summed up. How did we construct these reasonable principles could be found in Chapter 2. Our result implies that while there might be societies in which excluding some minorities from the delegation may work, but for most of the time, we should invite everyone to the table for a discussion that will be fruitful.

Chapter 3 mainly focuses on another important principle. It is called Participation Criterion, and it makes sure that no voter will be better off by abstaining, instead of voting. This is a formalization of a problem that is more visible in real life. First, there are non zero costs for voting, at least by walking into the building that everyone votes. Second, the probability that vote of someone affecting the outcome of the election is pretty small. So, there will be some people that will weight this "cost" against the benefit of positively affecting the result of the election, and decide not to vote. Even excluding such possibilities, there is the problem of possibly affecting the outcome in a negative way for themselves. While voting for their best candidate, in some election systems, you can easily make your worst candidate win, which is another cost that will be only visible ex-post, once the votes have been counted. 
The importance of this principle comes from the fact that democracy is by definition is a concept people should participate in. Trusting the wisdom of the crowd is not possible if no one is willing to voice their opinion. Thus, trying to analyze election systems, in which no one could make the outcome worse off for them at least, is an attempt to help the survival of democracy.

Finally the last chapter is about distances. In social choice theory, people are assumed to minimize some distance when preferring one ruler over the other. These distances has similar properties with distances between places, for example, just as Amsterdam is as close to Maastricht as Maastricht is close to Amsterdam, a ruler and a citizen are assumed to be equidistant from each other. In this last chapter, together with this property called symmetry, some other properties of metrics are analyzed, whether they are reasonable to expect in a setting where rulers are decision makers in an uncertain world. Intuitively, the effort is parallel to the concept of "social distance" from psychology which also is analyzed within other fields of economics. The result may be both useful for understanding the voter behavior better, which would help decision makers to be more informed about expectations. 



\section{Curriculum Vitae}

Emre Ergin was born on November 28, 1990 in Kayseri, Turkey. He obtained his Bachelor of Science in Economics together with a double major in Mathematics at Technology and Economics University, Ankara. He received his Masters of Arts degree in Economics at Bilkent University in 2014. Afterwards, he joined Maastricht University as a PhD candidate under the supervision of Prof. Dr. Arno Riedl, Prof. Dr. Hans Peters and Dr. Burak Can. The outcomes of his research are presented in this thesis. Some parts of this thesis have been presented at various international conferences.

His research interests are Social Choice Theory, Voting Theory, Mechanism Design. 
